\title{
Comparison of benzene, nitrobenzene and dinitrobenzene 2-arylsulfenylpyrroles
}

Jose R. Garabatos-Perera, Benjamin H. Rotstein and Alison Thompson*

Department of Chemistry, Dalhousie University. Halifax, Nova Scotia, B3H 4J3, Canada

alison.thompson@dal.ca

\section{Supporting Information}

\section{Table of Contents}

General experimental information .............................................................................. 3

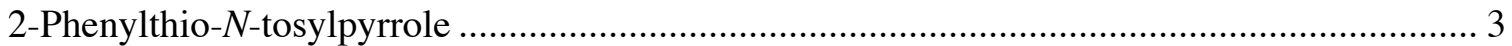

2-(4-Nitrobenzenesulfenyl)pyrrole (2) ........................................................................... 4

2-Benzenesulfinylpyrrole (4) …………………………………………………... 4

2-(4-Nitrobenzenesulfinyl)pyrrole (5) ………………………………………………... 5

General procedure (GP1) for formylation ................................................................. 5

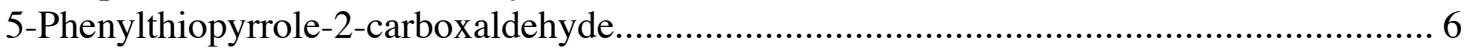

5-(4-Nitrobenzenesulfenyl)pyrrole-2-carboxaldehyde ………………................................ 6

5-(2,4-Dinitrobenzenesulfenyl)pyrrole-2-carboxaldehyde ………………………………….... 6

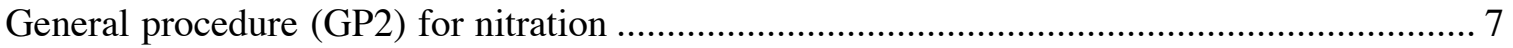

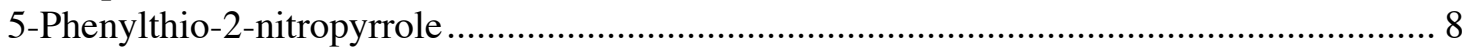

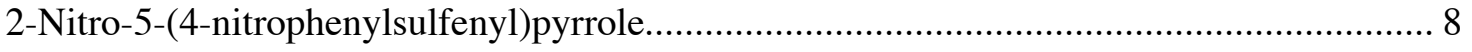

2-(2,4-Dinitrophenylsulfenyl)-5-nitropyrrole .................................................................. 8

General procedure (GP3) for acid-stability studies ............................................................. 9

General procedure for isomerization experiments (GP4) ..................................................... 9

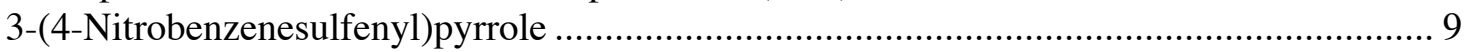

3-(2,4-Dinitrobenzenesulfenyl)pyrrole ……………………………………………..... 9

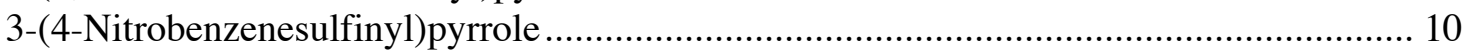

General procedure (GP5) for 1,9-bis(2,4-dinitrobenzenesulfenyl)dipyrromethanes................. 10

1,9-Bis(2,4-dinitrobenzenesulfenyl)-5-phenyldipyrromethane ……..................................... 11

1,9-Bis(2,4-dinitrobenzenesulfenyl)-5-(4-methylphenyl)dipyrromethane............................. 11

1,9-Bis(2,4-dinitrobenzenesulfenyl)-5-(4-nitrophenyl)dipyrromethane ................................ 12

1,9-Bis(2,4-dinitrobenzenesulfenyl)-5-(4-pyridyl)dipyrromethane ....................................... 12

1,9-Bis(2,4-dinitrobenzenesulfenyl)-5-(4-bromophenyl)dipyrromethane …….................... 13

Reductive desulfurization of 1,9-bis(2,4-dinitrobenzenesulfenyl)-5-phenyldipyrromethane... 13

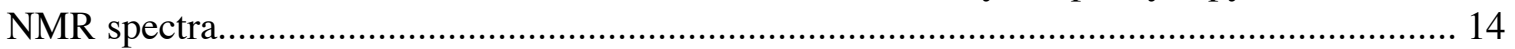

${ }^{13} \mathrm{C}$ spectrum, 5-(4-Nitrobenzenesulfenyl)-pyrrole-2-carboxaldehyde .................................. 14

DEPTQ135 spectrum, 5-(4-Nitrobenzenesulfenyl)-pyrrole-2-carboxaldehyde .................... 15

HMBC spectrum, 5-(4-Nitrobenzenesulfenyl)-pyrrole-2-carboxaldehyde ........................... 16

DEPTQ spectrum, 5-(2,4-Dinitrobenzenesulfenyl)pyrrole-2-carboxaldehyde...................... 19

HMQC spectrum, 5-(2,4-Dinitrobenzenesulfenyl)pyrrole-2-carboxaldehyde ...................... 20

HMBC, 5-(2,4-Dinitrobenzenesulfenyl)pyrrole-2-carboxaldehyde ……………………....... 21

${ }^{13} \mathrm{C}$ spectrum, 5-(2,4-Dinitrobenzenesulfenyl)pyrrole-3-carboxaldehyde ………………..... 22

DEPTQ135 spectrum, 5-(2,4-Dinitrobenzenesulfenyl)pyrrole-3-carboxaldehyde................. 23

HMQC spectrum, 5-(2,4-Dinitrobenzenesulfenyl)pyrrole-3-carboxaldehyde ...................... 24

HMBC spectrum, 5-(2,4-Dinitrobenzenesulfenyl)pyrrole-3-carboxaldehyde....................... 25

NOESY spectrum, 5-(2,4-Dinitrobenzenesulfenyl)pyrrole-3-carboxaldehyde ..................... 26

${ }^{13} \mathrm{C}$ NMR spectrum, 2-Phenylthio-5-nitropyrrole …………………………................... 27 


\section{Comparison of benzene, nitrobenzene and dinitrobenzene 2-arylsulfenylpyrroles}

Jose R. Garabatos-Perera, Benjamin H. Rotstein and Alison Thompson*

DEPTQ135 spectrum, 2-Phenylthio-5-nitropyrrole ........................................................... 28

HMQC NMR spectrum, 2-Phenylthio-5-nitropyrrole ……............................................. 29

HMBC NMR spectrum, 2-Phenylthio-5-nitropyrrole ...................................................... 30

${ }^{13} \mathrm{C}$ NMR spectrum, 2-Nitro-5-(4-nitrophenylsulfenyl)pyrrole ............................................ 31

DEPTQ135 NMR spectrum, 2-Nitro-5-(4-nitrophenylsulfenyl)pyrrole ………………….... 32

HMQC NMR spectrum, 2-Nitro-5-(4-nitrophenylsulfenyl)pyrrole ……………………...... 33

HMBC NMR spectrum, 2-Nitro-5-(4-nitrophenylsulfenyl)pyrrole ……………………….... 34

${ }^{13} \mathrm{C}$ NMR spectrum, 2-(2,4-Dinitrophenylsulfenyl)-5-nitropyrrole ....................................... 35

DEPTQ135 NMR spectrum, 2-(2,4-Dinitrophenylsulfenyl)-5-nitropyrrole ……………...... 36

HMQC NMR spectrum, 2-(2,4-Dinitrophenylsulfenyl)-5-nitropyrrole ................................ 37

HMBC NMR spectrum, 2-(2,4-Dinitrophenylsulfenyl)-5-nitropyrrole ................................ 38

Crude ${ }^{1} \mathrm{H}$ spectrum, 3-(4-Nitrobenzenesulfenyl)pyrrole and 2-(4-

Nitrobenzenesulfenyl)pyrrole (1:1) ............................................................................. 39

Crude ${ }^{13} \mathrm{C}$ spectrum, 3-(4-Nitrobenzenesulfenyl)pyrrole and 2-(4-

Nitrobenzenesulfenyl)pyrrole ....................................................................................... 40

Crude ${ }^{1} \mathrm{H}$ spectrum, 3-(2,4-Dinitrobenzenesulfenyl)pyrrole (major) and 2-(2,4-

Dinitrobenzenesulfenyl)pyrrole $(1: 4)$............................................................................. 41

Crude ${ }^{13} \mathrm{C}$ spectrum, 3-(2,4-Dinitrobenzenesulfenyl)pyrrole (major) and 2-(2,4-

Dinitrobenzenesulfenyl)pyrrole ................................................................................... 42

Crude ${ }^{1} \mathrm{H}$ spectrum, 3-(4-Nitrobenzenesulfinyl)pyrrole (major) and 2-(4-

Nitrobenzenesulfinyl)pyrrole (1:6)................................................................................ 43

Crude ${ }^{13} \mathrm{C}$ spectrum, 3-(4-Nitrobenzenesulfinyl)pyrrole (major) and 2-(4-

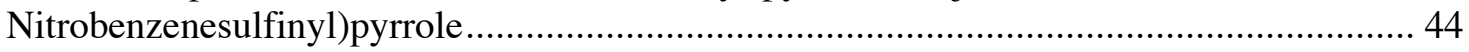

${ }^{13} \mathrm{C}$ NMR spectrum, 1,9-Bis(2,4-dinitrobenzenesulfenyl)-5-phenyldipyrromethane............. 45

DEPTQ135 spectrum, 1,9-Bis(2,4-dinitrobenzenesulfenyl)-5-phenyldipyrromethane......... 46

HMQC NMR spectrum, 1,9-Bis(2,4-dinitrobenzenesulfenyl)-5-phenyldipyrromethane..... 47

HMBC NMR spectrum, 1,9-Bis(2,4-dinitrobenzenesulfenyl)-5-phenyldipyrromethane ..... 48

${ }^{13} \mathrm{C}$ NMR spectrum, 1,9-Bis(2,4-dinitrobenzenesulfenyl)-5-(4-

methylphenyl)dipyrromethane

DEPTQ135 NMR spectrum, 1,9-Bis(2,4-dinitrobenzenesulfenyl)-5-(4-methylphenyl)-

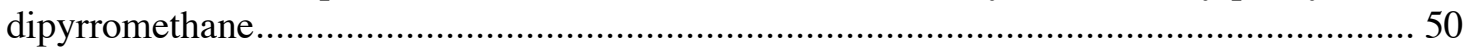

HMQC NMR spectrum, 1,9-Bis(2,4-dinitrobenzenesulfenyl)-5-(4-methylphenyl)-

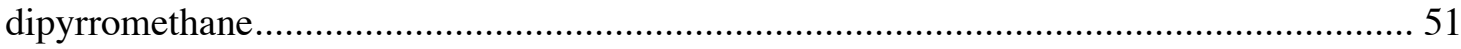

HMBC NMR spectrum, 1,9-Bis(2,4-dinitrobenzenesulfenyl)-5-(4-methylphenyl)-

dipyrromethane ................................................................................................ 52

${ }^{13} \mathrm{C}$ NMR spectrum, 1,9-Bis(2,4-dinitrobenzenesulfenyl)-5-(4-nitrophenyl)dipyrromethane

DEPTQ135 NMR spectrum, 1,9-Bis(2,4-dinitrobenzenesulfenyl)-5-(4-nitrophenyl)-

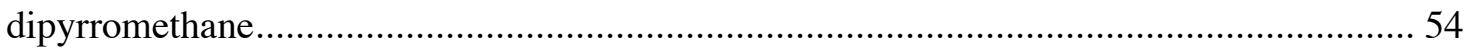

HMQC NMR spectrum, 1,9-Bis(2,4-dinitrobenzenesulfenyl)-5-(4-nitrophenyl)-

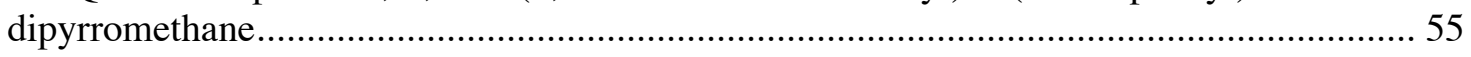

HMBC NMR spectrum, 1,9-Bis(2,4-dinitrobenzenesulfenyl)-5-(4-nitrophenyl)-

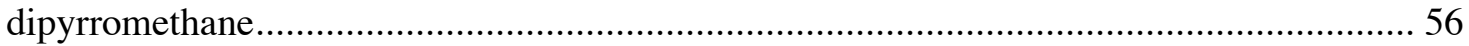

${ }^{13} \mathrm{C}$ NMR spectrum, 1,9-Bis(2,4-dinitrobenzenesulfenyl)-5-(4-

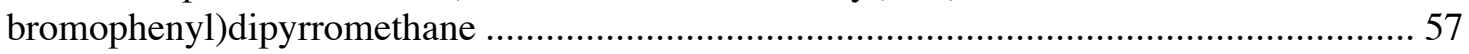

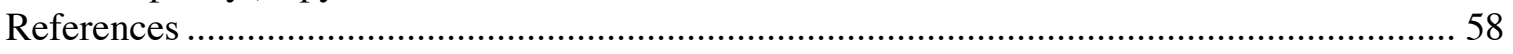




\section{Comparison of benzene, nitrobenzene and dinitrobenzene 2-arylsulfenylpyrroles}

Jose R. Garabatos-Perera, Benjamin H. Rotstein and Alison Thompson*

\section{General experimental information}

All ${ }^{1} \mathrm{H}$ NMR (500 MHz) and ${ }^{13} \mathrm{C}$ NMR $(125 \mathrm{MHz})$ chemical shifts are reported in ppm using tetramethylsilane $(0.00 \mathrm{ppm})$ or the solvent signal $\left[\mathrm{CDCl}_{3}\left({ }^{1} \mathrm{H} 7.26 \mathrm{ppm} ;{ }^{13} \mathrm{C} 71.23\right.\right.$ ppm), acetone-d6 ( $\left.{ }^{1} \mathrm{H} 2.05 \mathrm{ppm} ;{ }^{13} \mathrm{C} 29.84 \mathrm{ppm}\right)$, DMSO-d6 $\left({ }^{1} \mathrm{H} 2.5 \mathrm{ppm} ;{ }^{13} \mathrm{C} 39.52\right.$ ppm)] as internal reference. All coupling constants are reported in $\operatorname{Hertz}(\mathrm{Hz})$. Assignments were made using 2D and correlation spectroscopy (copies of spectra included). Mass spectra were obtained from sector (EI) and ion trap (ESI) instruments. All melting points are reported uncorrected. Column chromatography was performed using 230-400 mesh ultra pure silica. With the exclusion of solvents, chemicals were used as received. Dry, deoxygenated $\mathrm{CH}_{2} \mathrm{Cl}_{2}$ and tetrahydrofuran were obtained from a commercial solvent purification system. Phenylthiopyrrole (1), 2-2 $2,4-$ dinitrobenzenesulfenyl)pyrrole (3), ${ }^{3}$ and 2-(2,4-dinitrobenzenesulfinyl)pyrrole $(\mathbf{6})^{3}$ were prepared as previously reported. CAUTION: In our hands, 2-thiocyanatopyrrole (precursor to 1) decomposed very readily and was also extremely liable to pass through multiple layers of latex gloves to cause stains and discomfort of the skin. Furthermore, in our hands 1 was unstable in air and was thus protected as its $N$-tosyl derivative, ${ }^{4}$ for ease of manipulation, and then deprotected with base immediately before use. ${ }^{5}$

\section{2-Phenylthio- $N$-tosylpyrrole}

Prepared by a modification of a previously reported procedure: ${ }^{4}$ a suspension of 2phenylthiopyrrole (1) $(1.95 \mathrm{~g}, 11.0 \mathrm{mmol})$ and $\mathrm{NaOH}(1.8 \mathrm{~g}, 44 \mathrm{mmol})$ in $\mathrm{ClCH}_{2} \mathrm{CH}_{2} \mathrm{Cl}$ $(70 \mathrm{~mL})$ was treated at $25{ }^{\circ} \mathrm{C}$ with tosyl chloride $(2.5 \mathrm{~g}, 13.2 \mathrm{mmol})$. The resulting mixture was stirred for $12 \mathrm{~h}$ at $25{ }^{\circ} \mathrm{C}$ under $\mathrm{N}_{2}$. The reaction was then treated with $\mathrm{H}_{2} \mathrm{O}$ $(150 \mathrm{~mL})$, and the mixture extracted with $\mathrm{CH}_{2} \mathrm{Cl}_{2}(3 \times 100 \mathrm{~mL})$. The combined organic 


\section{Comparison of benzene, nitrobenzene and dinitrobenzene 2-arylsulfenylpyrroles}

Jose R. Garabatos-Perera, Benjamin H. Rotstein and Alison Thompson*

layers were washed sat. aq $\mathrm{NaCl}(3 \times 100 \mathrm{~mL})$, dried over $\mathrm{MgSO}_{4}$, and evaporated to give the title compound $(3.1 \mathrm{~g}, 9.4 \mathrm{mmol}, 85 \%)$ as a grey solid identified by comparison with an authentic sample ( ${ }^{1} \mathrm{H}$ NMR). ${ }^{5} \mathrm{MS}(\mathrm{EI}) \mathrm{m} / \mathrm{z} 329\left(\mathrm{M}^{+}, 23\right), 174\left(\mathrm{M}^{+}-\mathrm{Tos}, 100\right)$; $\operatorname{HRMS}\left(\mathrm{EI}, \mathrm{C}_{17} \mathrm{H}_{15} \mathrm{NO}_{2} \mathrm{~S}_{2}\right.$ ) calcd. 329.0544, found 329.0543.

\section{2-(4-Nitrobenzenesulfenyl)pyrrole (2)}

Prepared by a modification of a previously reported procedure: ${ }^{3}$ pyrrole $(0.2 \mathrm{~mL}, 2.7$ mmol) was added to a solution of 4-nitrobenzenesulfenyl chloride (0.5 g, $2.6 \mathrm{mmol})$ in dry $\mathrm{CH}_{2} \mathrm{Cl}_{2}(25 \mathrm{~mL})$ at $25{ }^{\circ} \mathrm{C}$ under $\mathrm{N}_{2}$. The reaction mixture was stirred for $20 \mathrm{~h}$ at $25{ }^{\circ} \mathrm{C}$. The reaction was then treated with $\mathrm{Et}_{3} \mathrm{~N}(5 \mathrm{~mL})$ and the solvent removed in vacuo. Column chromatography $\left(\mathrm{SiO}_{2}, 2.5 \times 15 \mathrm{~cm}\right.$, hexanes: $\mathrm{CH}_{2} \mathrm{Cl}_{2}$ 1:1) gave the title compound $(0.53 \mathrm{~g}, 93 \%)$, as a pale-yellow solid identified by comparison with an authentic sample $\left({ }^{1} \mathrm{H}\right.$ NMR). ${ }^{3}$

\section{2-Benzenesulfinylpyrrole (4)}

Prepared by a modification of a previously reported procedure: ${ }^{3}$ a solution of phenylthiopyrrole (1) $(0.10 \mathrm{~g}, 0.6 \mathrm{mmol})$ and $m$-CPBA $(0.189 \mathrm{~g}, 0.55 \mathrm{mmol})$ in dry $\mathrm{CH}_{2} \mathrm{Cl}_{2}(6 \mathrm{~mL})$ was stirred under $\mathrm{N}_{2}$ at $0{ }^{\circ} \mathrm{C}$ for $25 \mathrm{~min}$. To the resulting suspension sat. aq $\mathrm{NaHCO}_{3}(25 \mathrm{~mL})$ was added at $0{ }^{\circ} \mathrm{C}$. The organic layer was diluted with EtOAc $(50 \mathrm{~mL})$, washed with sat. aq $\mathrm{NaHCO}_{3}(3 \times 100 \mathrm{~mL})$, brine $(3 \times 100 \mathrm{~mL})$, dried over $\mathrm{MgSO}_{4}$, and the solvent was then removed under reduced pressure. Purification using column chromatography $\left(\mathrm{SiO}_{2}, 2.5 \times 10 \mathrm{~cm}, \mathrm{CH}_{2} \mathrm{Cl}_{2}\right.$ :EtOAc 4:2) gave the title compound $(0.086$ $\mathrm{g}, 79 \%)$ as a white solid which was identified by comparison with an authentic sample $\left({ }^{1} \mathrm{H}\right.$ NMR $) .{ }^{6}$ 
Comparison of benzene, nitrobenzene and dinitrobenzene 2-arylsulfenylpyrroles

Jose R. Garabatos-Perera, Benjamin H. Rotstein and Alison Thompson*

\section{2-(4-Nitrobenzenesulfinyl)pyrrole (5)}

Prepared by a modification of a previously reported procedure: ${ }^{3}$ a solution of 2-(4nitrobenzenesulfenyl)pyrrole $(3)(0.25 \mathrm{~g}, 1.1 \mathrm{mmol})$ and $m$-CPBA $(0.431 \mathrm{~g}, 1.2 \mathrm{mmol})$ in dry $\mathrm{CH}_{2} \mathrm{Cl}_{2}(15 \mathrm{~mL})$ was stirred under $\mathrm{N}_{2}$ at $25{ }^{\circ} \mathrm{C}$ for $2 \mathrm{~h}$. To the resulting suspension, sat. aq $\mathrm{NaHCO}_{3}(25 \mathrm{~mL})$ was added at $25^{\circ} \mathrm{C}$. The organic layer was diluted with $\mathrm{CH}_{2} \mathrm{Cl}_{2}$ (25 mL), washed with sat. aq $\mathrm{NaHCO}_{3}(3 \times 25 \mathrm{~mL})$, brine $(3 \times 25 \mathrm{~mL})$, dried over $\mathrm{MgSO}_{4}$, and the solvent was then removed under reduced pressure. Purification using column chromatography $\left(\mathrm{SiO}_{2}, 2.5 \times 15 \mathrm{~cm}, \mathrm{CH}_{2} \mathrm{Cl}_{2}\right)$ gave the title compound $(0.126 \mathrm{~g}$, $47 \%)$ as a white solid which was identified by comparison with an authentic sample $\left({ }^{1} \mathrm{H}\right.$ NMR). ${ }^{3}$

\section{General procedure (GP1) for formylation}

To DMF (1.5 eq) at $0{ }^{\circ} \mathrm{C}$ under $\mathrm{N}_{2}, \mathrm{POCl}_{3}(1.5 \mathrm{eq})$ was added drop-wise. After stirring at $100{ }^{\circ} \mathrm{C}$ for $15 \mathrm{~min}$ (to ensure the complete formation of the phosphorus oxychloridedimethylformamide complex), a solution of the pyrrole (1.0 eq) in 1,2dichloroethane was added at $0{ }^{\circ} \mathrm{C}$. The reaction mixture was heated at reflux for the appropriate amount of time (1.0-1.5 h) and then hydrolysis was performed by the addition of an aqueous solution of $\mathrm{NaOAc}$. The reaction mixture was then stirred at reflux temperature for $1 \mathrm{~h}$, before being allowed to cool and then extracted three times with ether. The combined organic layers were washed three times with sat. $\mathrm{Na}_{2} \mathrm{CO}_{3}$ (aq) and once with brine, before being dried over $\mathrm{MgSO}_{4}$ and filtered. The solvent was removed under reduced pressure and the crude product was purified using column chromatography. 
Comparison of benzene, nitrobenzene and dinitrobenzene 2-arylsulfenylpyrroles

Jose R. Garabatos-Perera, Benjamin H. Rotstein and Alison Thompson*

\section{5-Phenylthiopyrrole-2-carboxaldehyde}

GP1: DMF (0.14 mL, $1.8 \mathrm{mmol}) ; \mathrm{POCl}_{3}(0.18 \mathrm{~mL}, 1.8 \mathrm{mmol})$; phenylthiopyrrole (1)

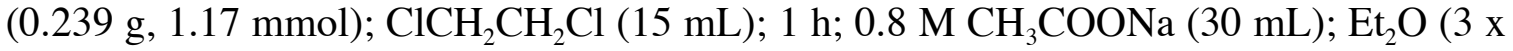
$25 \mathrm{~mL})$; sat. $\mathrm{Na}_{2} \mathrm{CO}_{3}(3 \times 50 \mathrm{~mL})$; sat. $\mathrm{NaCl}$; column chromatography $\left(\mathrm{SiO}_{2}, 15 \times 2.5 \mathrm{~cm}\right.$, hexanes: $\mathrm{CH}_{2} \mathrm{Cl}_{2}$ 1:3) gave the title compound $(0.209 \mathrm{~g}, 75 \%)$, as a white solid which was identified by comparison with an authentic sample prepared by an alternative route $\left({ }^{1} \mathrm{H} \mathrm{NMR}\right){ }^{7}$

\section{5-(4-Nitrobenzenesulfenyl)pyrrole-2-carboxaldehyde}

GP1: DMF (0.1 mL, $1.4 \mathrm{mmol})$; $\mathrm{POCl}_{3}(0.13 \mathrm{~mL}, 1.4 \mathrm{mmol}) ;$ 2-(4-nitrobenzenesulfenyl)pyrrole (2) (0.200 g, $0.9 \mathrm{mmol}) ; \mathrm{ClCH}_{2} \mathrm{CH}_{2} \mathrm{Cl}(15 \mathrm{~mL}) ; 1 \mathrm{~h} ; 0.6 \mathrm{M} \mathrm{CH}_{3} \mathrm{COONa}(30 \mathrm{~mL})$; $1 \mathrm{~h} ; \mathrm{Et}_{2} \mathrm{O}(3 \times 25 \mathrm{~mL})$; sat. $\mathrm{Na}_{2} \mathrm{CO}_{3}(3 \times 25 \mathrm{~mL})$; column chromatography $\left(\mathrm{SiO}_{2}, 2.5 \times 15\right.$ $\left.\mathrm{cm}, \mathrm{CH}_{2} \mathrm{Cl}_{2}\right)$ gave the title compound $(0.171 \mathrm{~g}, 76 \%)$ as a white solid. mp $176-179{ }^{\circ} \mathrm{C} ;{ }^{1} \mathrm{H}$ NMR (DMSO-d6) $\delta 12.92(\mathrm{~s}, 1 \mathrm{H}, \mathrm{NH}), 9.57$ (s, 1H, CHO), 8.14 (d, J = 9.7, 2H, 8(10)H), $7.23(\mathrm{~d}, J=9.7,2 \mathrm{H}, 7(11)-\mathrm{H}), 7.15(\mathrm{~d}, J=3.8,1 \mathrm{H}, 3-\mathrm{H}$ or $4-\mathrm{H}), 6.68(\mathrm{~d}, J=3.8,1 \mathrm{H}$, 3-H or 4-H); ${ }^{13} \mathrm{C}$ NMR (DMSO-d6) $\delta 179.8$ (CHO), 147.0, 145.2, 136.3, 126.1 [C-7(11)], 124.3 [C-8(10)], 122.8 (C-3 or C-4), 120.6, 119.7 (C-3 or C-4); MS (ESI+) m/z $247\left(\mathrm{M}^{+}\right.$, 100), 219 (20); HRMS(EI+, $\left.\mathrm{C}_{11} \mathrm{H}_{8} \mathrm{~N}_{2} \mathrm{O}_{3} \mathrm{~S}\right)$ calcd. 248.0256, found 248.0259.

\section{5-(2,4-Dinitrobenzenesulfenyl)pyrrole-2-carboxaldehyde}

GP1: DMF (0.09 mL, $1.1 \mathrm{mmol}) ; \mathrm{POCl}_{3}(0.10 \mathrm{~mL}, 1.1 \mathrm{mmol}) ; 2-(2,4-$ dinitrobenzenesulfenyl)pyrrole (3) (0.200 g, $0.75 \mathrm{mmol})$; $\mathrm{ClCH}_{2} \mathrm{CH}_{2} \mathrm{Cl}(20 \mathrm{~mL}) ; 1.5 \mathrm{~h}$; $0.6 \mathrm{M} \mathrm{CH}_{3} \mathrm{COONa}(25 \mathrm{~mL}) ; 1 \mathrm{~h} ; \mathrm{Et}_{2} \mathrm{O}(3 \times 25 \mathrm{~mL}) ; \mathrm{Na}_{2} \mathrm{CO}_{3}(3 \times 25 \mathrm{~mL})$; Column chromatography $\left(\mathrm{SiO}_{2}, 2.5 \times 15 \mathrm{~cm}, \mathrm{CH}_{2} \mathrm{Cl}_{2} \rightarrow \mathrm{CH}_{2} \mathrm{Cl}_{2}\right.$ :EtOAc 9:1) gave the title 
Comparison of benzene, nitrobenzene and dinitrobenzene 2-arylsulfenylpyrroles

Jose R. Garabatos-Perera, Benjamin H. Rotstein and Alison Thompson*

compound $(0.147 \mathrm{~g}, 67 \%)$ as a yellow solid, and 2-(2,4-dinitrophenylsulfenyl)pyrrole-3carboxyaldehyde $(0.005 \mathrm{~g}, 2.5 \%)$, as a red solid.

5-(2,4-Dinitrobenzenesulfenyl)pyrrole-2-carboxaldehyde: $\mathrm{mp} \quad 212-214{ }^{\circ} \mathrm{C} ;{ }^{1} \mathrm{H}$ NMR (DMSO-d6) $\delta 12.95$ (s, 1H, NH), 9.62 (s, 1H, CHO), 8.9 (d, $J=2.4,1 \mathrm{H}, 8-\mathrm{H}), 8.4$ (dd, $J$ $=2.5, J=9,1 \mathrm{H}, 10-\mathrm{H}), 7.2(\mathrm{dd}, J=3.8,1 \mathrm{H}, 3-\mathrm{H}$ or $4-\mathrm{H}), 7.0(\mathrm{~d}, J=9,1 \mathrm{H}, 11-\mathrm{H}), 6.79$ $(\mathrm{d}, J=3.8,1 \mathrm{H}, 3-\mathrm{H}$ or $4-\mathrm{H}) ;{ }^{13} \mathrm{C}$ NMR (DMSO-d6) $\delta 180.1(\mathrm{CHO}), 145.4,144.7,143.8$, 136.9, 129.2, 128.2, 122.1, 121.2, 120.8, 120.6; MS(ESI+) m/z 292 (M+, 100), 245 (31); HRMS (EI+, $\mathrm{C}_{11} \mathrm{H}_{7} \mathrm{~N}_{3} \mathrm{O}_{5} \mathrm{~S}$ ) calcd. 293.0106, found 293.0091.

5-(2,4-Dinitrophenylsulfenyl)pyrrole-3-carboxaldehyde: mp 87-88 ${ }^{\circ} \mathrm{C} ;{ }^{1} \mathrm{H}$ NMR (DMSOd6) $\delta 12.57(1 \mathrm{H}, \mathrm{s}, \mathrm{NH}), 9.77(1 \mathrm{H}, \mathrm{s}, \mathrm{CHO}), 8.90(\mathrm{~d}, J=2.45,1 \mathrm{H}, 8-\mathrm{H}), 8.42(\mathrm{dd}, J=2.5$, $J=9,1 \mathrm{H}, 10-\mathrm{H}), 8.05(\mathrm{dd}, J=1.6, J=3.25,1 \mathrm{H}, 2-\mathrm{H}$ or $4-\mathrm{H}), 7.07(\mathrm{dd}, J=1.95=1.95$, 1H, 2-H or 4-H), $7.00(\mathrm{~d}, J=9,1 \mathrm{H}, 11-\mathrm{H}) ;{ }^{13} \mathrm{C}$ NMR (DMSO-d6) $\delta 185.0(C \mathrm{HO}), 146.0$, $144.7,143.6,133.0,128.8,128.2,127.8,121.3,118.3,115.9$.

\section{General procedure (GP2) for nitration}

A solution of the pyrrole $(1.0 \mathrm{eq})$ in $\mathrm{Ac}_{2} \mathrm{O}$ was treated with a cold solution $\left(0{ }^{\circ} \mathrm{C}\right)$ of $70 \%$ $\mathrm{HNO}_{3}(1.1 \mathrm{eq})$ in $\mathrm{Ac}_{2} \mathrm{O}$. After stirring at the appropriate temperature $\left(0-25^{\circ} \mathrm{C}\right)$ for the appropriate amount of time $(0.5-1.0 \mathrm{~h})$, the reaction mixture was poured onto ice, and treated with solid $\mathrm{Na}_{2} \mathrm{CO}_{3}$ until $\mathrm{pH}$ 7. Extraction of the aqueous solution three times with ether, followed by washing of the combined organic fractions with brine until $\mathrm{pH} 7$, drying over $\mathrm{MgSO}_{4}$, filtration, and removal of the solvent under reduced pressure gave the crude product which was purified using column chromatography. 
Comparison of benzene, nitrobenzene and dinitrobenzene 2-arylsulfenylpyrroles

Jose R. Garabatos-Perera, Benjamin H. Rotstein and Alison Thompson*

\section{5-Phenylthio-2-nitropyrrole}

GP2: phenylthiopyrrole (1) $(0.231 \mathrm{~g}, 1.3 \mathrm{mmol}) ; \mathrm{HNO}_{3}(0.06 \mathrm{~mL}, 1.43 \mathrm{mmol}) ; \mathrm{Ac}_{2} \mathrm{O}(20$ $\mathrm{mL}) ; 0{ }^{\circ} \mathrm{C} ; 30 \mathrm{~min} ; \mathrm{Et}_{2} \mathrm{O}(3 \times 25 \mathrm{~mL})$; column chromatography $\left(\mathrm{SiO}_{2}, 2.5 \times 15 \mathrm{~cm}\right.$, $\mathrm{CH}_{2} \mathrm{Cl}_{2}$ :hexanes $\left.1: 1\right)$ gave the title compound $(0.053 \mathrm{~g}, 18 \%)$ as a yellow-brown liquid. ${ }^{1} \mathrm{H}$ NMR $\left(\mathrm{CDCl}_{3}\right) \delta 9.38$ (br s, 1H, NH), 7.33-7.11 (m, 5H, Ar), 7.10 (d, 1H, J = 4.15, 3$\mathrm{H}$ or $4-\mathrm{H}), 6.43(\mathrm{~d}, 1 \mathrm{H}, J=4.2,3-\mathrm{H}$ or $4-\mathrm{H}) ;{ }^{13} \mathrm{C} \mathrm{NMR}\left(\mathrm{CDCl}_{3}\right) \delta 138.5,133.48,129.91$, $129.90,128.14,127.69,117.38,112.21$.

\section{2-Nitro-5-(4-nitrophenylsulfenyl)pyrrole}

GP2: 2-(4-nitrobenzenesulfenyl)pyrrole (2) (0.094 g, $0.425 \mathrm{mmol}) ; \mathrm{HNO}_{3}(0.03 \mathrm{~mL}$, $0.468 \mathrm{mmol}) ; \mathrm{Ac}_{2} \mathrm{O}(25 \mathrm{~mL}) ; 0{ }^{\circ} \mathrm{C}$ to $25{ }^{\circ} \mathrm{C} ; 1 \mathrm{~h} ; \mathrm{Et}_{2} \mathrm{O}(3 \times 40 \mathrm{~mL})$; sat. aq $\mathrm{NaCl}(3 \times 25$ $\mathrm{mL})$; column chromatography $\left(\mathrm{SiO}_{2}, 2.5 \times 10 \mathrm{~cm}, \mathrm{CH}_{2} \mathrm{Cl}_{2}\right)$ gave the title compound (0.0182 g, $16 \%$ ), as a white solid. mp 138-139 ${ }^{\circ}$; ${ }^{1} \mathrm{H}$ NMR (DMSO-d6) $\delta 13.99$ (s, 1H, $\mathrm{NH}), 8.15[\mathrm{~d}, J=9.05,2 \mathrm{H}, 8(10)-\mathrm{H}], 7.32[\mathrm{~d}, J=9.05,2 \mathrm{H}, 7(11)-\mathrm{H}], 7.27(\mathrm{~d}, J=4.1$, $1 \mathrm{H}, 3-\mathrm{H}$ or $4-\mathrm{H}), 6.71(\mathrm{~d}, J=4.1,1 \mathrm{H}, 3-\mathrm{H}$ or $4-\mathrm{H}) ;{ }^{13} \mathrm{C}$ NMR (DMSO-d6) $\delta 146.05$, 145.46, 139.8, 126.5 [C-7(11)], 124.3 [C-8(10)], 122.0, 119.8, 112.1; MS (EI+) m/z 265 $\left(\mathrm{M}^{+}, 100\right)$; HRMS (EI+, $\left.\mathrm{C}_{10} \mathrm{H}_{7} \mathrm{~N}_{3} \mathrm{O}_{4} \mathrm{~S}\right)$ calcd. 265.0157, found 265.0154.

\section{2-(2,4-Dinitrophenylsulfenyl)-5-nitropyrrole}

GP2: 2-(2,4-dinitrobenzenesulfenyl)-pyrrole (5) (0.1 g, $0.377 \mathrm{mmol}) ; \mathrm{HNO}_{3}(0.026 \mathrm{~mL}$, $0.414 \mathrm{mmol}) ; \mathrm{Ac}_{2} \mathrm{O}(10 \mathrm{~mL}) ; 0{ }^{\circ} \mathrm{C}$ to $25^{\circ} \mathrm{C} ; 1 \mathrm{~h} ; \mathrm{Et}_{2} \mathrm{O}(3 \times 40 \mathrm{~mL})$; sat. $\mathrm{NaCl}(3 \times 25 \mathrm{~mL})$; column chromatography $\left(\mathrm{SiO}_{2}, 2.5 \times 10 \mathrm{~cm}\right.$, hexanes: $\mathrm{CH}_{2} \mathrm{Cl}_{2}$ 1:4) gave the title compound $(0.041 \mathrm{~g}, 35 \%)$ as a yellow solid. mp $140-142{ }^{\circ} \mathrm{C} ;{ }^{1} \mathrm{H}$ NMR $\left(\mathrm{CD}_{2} \mathrm{Cl}_{2}\right) \delta 9.78$ (br s, $1 \mathrm{H}, \mathrm{NH}), 9.08(\mathrm{~d}, J=2.5,1 \mathrm{H}, 8-\mathrm{H}), 8.23-8.25(\mathrm{dd}, J=9, J=2.5,1 \mathrm{H}, 10-\mathrm{H}), 7.24$ - $7.25(\mathrm{~m}, 1 \mathrm{H}, 3-\mathrm{H}$ or $4-\mathrm{H}), 7.05-7.08(\mathrm{~d}, J=9,1 \mathrm{H}, 11-\mathrm{H}), 6.76-6.77(\mathrm{~m}, 1 \mathrm{H}, 3-\mathrm{H}$ or $4-$ 
Comparison of benzene, nitrobenzene and dinitrobenzene 2-arylsulfenylpyrroles

Jose R. Garabatos-Perera, Benjamin H. Rotstein and Alison Thompson*

$\mathrm{H}) ;{ }^{13} \mathrm{C}$ NMR $\left(\mathrm{CD}_{2} \mathrm{Cl}_{2}\right) \delta 128.95,127.86,121.93,121.61,111.81 ; \mathrm{MS}(\mathrm{APCI}+) \mathrm{m} / z .309$

$\left(\mathrm{M}^{+}, 100\right)$.

\section{General procedure (GP3) for acid-stability studies}

Pyrrole $(53 \mu \mathrm{mol})$ and 1,4-dichlorobenzene $(20 \mu \mathrm{mol}, 0.003 \mathrm{~g})$ were dissolved in $\mathrm{CD}_{2} \mathrm{Cl}_{2}$ $(600 \mu \mathrm{L})$. The solution was then transferred, with filtration if necessary, into an NMR tube and ${ }^{1} \mathrm{H}$ NMR spectra were acquired every 30 minutes for a duration of 2.5 hours. Following the acquisition of each of the first five spectra, $\mathrm{CF}_{3} \mathrm{CO}_{2} \mathrm{D}(4.1 \mu \mathrm{L}, 53 \mu \mathrm{mol})$ was added to the tube, followed by thorough mixing via shaking.

\section{General procedure for isomerization experiments (GP4)}

A solution of the pyrrole (1.0 eq.) in a 1:1 mixture of TFA (37 eq.) and $\mathrm{ClCH}_{2} \mathrm{CH}_{2} \mathrm{Cl}$, was stirred at the appropriate temperature $\left(25^{\circ} \mathrm{C}\right.$ or $\left.84{ }^{\circ} \mathrm{C}\right)$ for $2 \mathrm{~h}$. After removal of the solvent in vacuo, analysis of the mixture using ${ }^{1} \mathrm{H}$ NMR spectroscopy gave the relative ratio of 2- and 3-isomers.

\section{3-(4-Nitrobenzenesulfenyl)pyrrole}

GP4: 2-(4-nitrobenzenesulfenyl)pyrrole (2) (0.05 g, $0.23 \mathrm{mmol})$; TFA (0.6 mL, 8.4 mmol); $\mathrm{ClCH}_{2} \mathrm{CH}_{2} \mathrm{Cl}(0.6 \mathrm{~mL}) ; 25{ }^{\circ} \mathrm{C} ; 2 \mathrm{~h} ; 1: 1$ mixture of 2- and 3-(4dinitrobenzenesulfenyl)pyrrole. ${ }^{1} \mathrm{H}$ NMR (DMSO-d6) $\delta 11.64(\mathrm{~s}, 1 \mathrm{H}, \mathrm{NH}), 8.08$ (d, $J=$ $8.9,1 \mathrm{H}, 10-\mathrm{H}), 7.19-7.12(\mathrm{~m}, 1 \mathrm{H}, 2-\mathrm{H}$ or $5-\mathrm{H}), 7.20(\mathrm{~d}, J=8.9,1 \mathrm{H}, 11-\mathrm{H}), 7.02-7.00(\mathrm{~m}$,

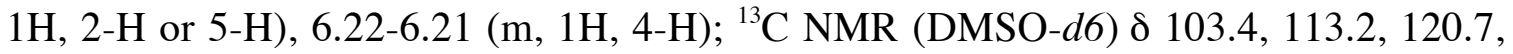
$125.0,124.6,124.9,144.2,151.5$.

\section{3-(2,4-Dinitrobenzenesulfenyl)pyrrole}

GP4: 2-(2,4-dinitrobenzenesulfenyl)pyrrole (3) (0.1 g, $0.4 \mathrm{mmol})$; TFA (1.0 mL, 13.1 mmol); $\mathrm{ClCH}_{2} \mathrm{CH}_{2} \mathrm{Cl}(1.0 \mathrm{~mL}) ; 84{ }^{\circ} \mathrm{C} ; 2 \mathrm{~h} ; 1: 4$ inseparable mixture of 2- and 3-(2,4- 
Comparison of benzene, nitrobenzene and dinitrobenzene 2-arylsulfenylpyrroles Jose R. Garabatos-Perera, Benjamin H. Rotstein and Alison Thompson*

dinitrobenzenesulfenyl)pyrrole. ${ }^{1} \mathrm{H}$ NMR (DMSO-d6) $\delta 11.64(\mathrm{~s}, 1 \mathrm{H}, \mathrm{NH}), 8.83$ (d, $J=$ $2.5,1 \mathrm{H}, 8-\mathrm{H}), 8.34(\mathrm{dd}, J=2.5, J=9,1 \mathrm{H}, 10-\mathrm{H}), 7.25-7.23(\mathrm{~m}, 1 \mathrm{H}, 2-\mathrm{H}$ or $5-\mathrm{H}), 7.22(\mathrm{~d}$, $J=9,1 \mathrm{H}, 11-\mathrm{H}), 7.08-7.07(\mathrm{~m}, 1 \mathrm{H}, 2-\mathrm{H}$ or $5-\mathrm{H}), 6.23-6.22(\mathrm{~m}, 1 \mathrm{H}, 4-\mathrm{H}) ;{ }^{13} \mathrm{C} \mathrm{NMR}$ (DMSO-d6) $\delta 149.7,143.7,143.0,128.6,127.3,125.7,121.4,121.0,113.0,103.4$.

\section{3-(4-Nitrobenzenesulfinyl)pyrrole}

GP4: 2-(4-nitrobenzenesulfinyl)pyrrole (5) (0.025 g, $0.1 \mathrm{mmol})$; TFA (0.3 mL, 3.8 mmol); $\mathrm{ClCH}_{2} \mathrm{CH}_{2} \mathrm{Cl}(0.3 \mathrm{~mL}) ; 25{ }^{\circ} \mathrm{C} ; 2 \mathrm{~h} ; 1: 6$ mixture of 2- and 3-(4nitrobenzenesulfinyl)pyrrole.

GP4: 2-(4-nitrobenzenesulfinyl)pyrrole (5) (0.025 g, $0.1 \mathrm{mmol})$; TFA (0.3 mL, 3.8 mmol); $\mathrm{ClCH}_{2} \mathrm{CH}_{2} \mathrm{Cl}(0.3 \mathrm{~mL}) ; 84{ }^{\circ} \mathrm{C} ; 2 \mathrm{~h} ; 1: 5$ mixture of 2- and 3-(4nitrobenzenesulfinyl)pyrrole. ${ }^{1} \mathrm{H}$ NMR (DMSO- $\left.d 6\right) \delta 11.56(\mathrm{~s}, 1 \mathrm{H}, \mathrm{NH}), 8.35(\mathrm{~d}, J=6.9$, $1 \mathrm{H}, 10-\mathrm{H}), 7.82(\mathrm{~d}, J=6.85,1 \mathrm{H}, 11-\mathrm{H}), 7.51-7.50(\mathrm{~m}, 1 \mathrm{H}, 2-\mathrm{H}$ or $5-\mathrm{H}), 6.93-6.89(\mathrm{~m}$, 1H, 2-H or 5-H), 6.04-6.03 (m, 1H, 4-H); ${ }^{13} \mathrm{C}$ NMR (DMSO-d6) $\delta$ 105.8, 121.3, 122.7, 124.1, 126.0, 128.0, 148.4, 153.9.

\section{General procedure (GP5) for 1,9-bis(2,4-dinitrobenzenesulfenyl)dipyrromethanes}

A solution of 2-(2,4-dinitrobenzenesulfenyl)pyrrole (3) (2 eq), aldehyde (1 eq) and TFA in degassed $\mathrm{CH}_{2} \mathrm{Cl}_{2}$ was stirred at reflux temperature for the appropriate amount of time (20-48 h). To the resulting mixture, $0.1 \mathrm{~N} \mathrm{NaOH}(50 \mathrm{~mL})$ was added at $25{ }^{\circ} \mathrm{C}$. The organic phase was washed with $0.1 \mathrm{~N} \mathrm{NaOH}(3 \times 50 \mathrm{~mL})$ and $\mathrm{H}_{2} \mathrm{O}(3 \times 50 \mathrm{~mL})$ before being dried over $\mathrm{MgSO}_{4}$ and filtered. The solvent was removed under reduced pressure to give the crude product which was purified using column chromatography. 
Comparison of benzene, nitrobenzene and dinitrobenzene 2-arylsulfenylpyrroles

Jose R. Garabatos-Perera, Benjamin H. Rotstein and Alison Thompson*

\section{1,9-Bis(2,4-dinitrobenzenesulfenyl)-5-phenyldipyrromethane}

GP5: 2-(2,4-dinitrobenzenesulfenyl)pyrrole (3) (0.326 g, $1.2 \mathrm{mmol})$; benzaldehyde (0.05 $\mathrm{mL}, 0.49 \mathrm{mmol})$; TFA (0.1 mL, $1.3 \mathrm{mmol}) ; \mathrm{CH}_{2} \mathrm{Cl}_{2}(25 \mathrm{~mL}) ; 20 \mathrm{~h}$; column chromatography $\left(\mathrm{SiO}_{2}, 3\right.$ x $5 \mathrm{~cm}$, hexane: $\left.\mathrm{CH}_{2} \mathrm{Cl}_{2} 2: 8\right)$ gave the title compound $(0.179 \mathrm{~g}$, $59 \%$ ), as a yellow-red solid. mp 132-135 ${ }^{\circ} \mathrm{C} ;{ }^{1} \mathrm{H}$ NMR (DMSO-d6) $\delta 11.59$ (s, 2H, NH), $8.79[\mathrm{~d}, J=2.4,2 \mathrm{H}, 18(24)-\mathrm{H}], 8.18[\mathrm{dd}, J=2.45, J=9.05,2 \mathrm{H}, 20(26)-\mathrm{H}], 7.37[\mathrm{dd}, J=$ $7.2=7.2,2 \mathrm{H}, 12(14)-\mathrm{H}], 7.28[\mathrm{~d}, J=7.95,2 \mathrm{H}, 11(15)-\mathrm{H}], 7.28(\mathrm{~m}, 1 \mathrm{H}, \mathrm{H}-13), 6.92[\mathrm{~d}, J$ $=9.15,2 \mathrm{H}, 21(27)-\mathrm{H}], 6.54[\mathrm{dd}, J=2.8=2.8,2 \mathrm{H}, 2(8)-\mathrm{H}$ or $3(7)-\mathrm{H}], 5.98[\mathrm{dd}, J=2.6=$ 2.6, 2H, 2(8)-H or 3(7)-H], 5.55 (s, 1H, H-5); ${ }^{13} \mathrm{C}$ NMR (DMSO-d6, TMS) $\delta 149.1$, $144.6,143.6,132.1,139.9,129.5,128.9,128.7,127.8,127.3,121.4,120.3,111.1,110.5$, $44.2(\mathrm{CH}, \mathrm{C}-5)$; MS(ESI+) m/z $640.1\left(\mathrm{M}^{+}+\mathrm{Na}, 20\right), 1258.5\left(2 \mathrm{M}^{+}+\mathrm{Na}, 65\right)$; HRMS (ESI+, $\mathrm{C}_{27} \mathrm{H}_{18} \mathrm{~N}_{6} \mathrm{NaO}_{8} \mathrm{~S}_{2}$ ) calcd. 641.0520, found 641.0520.

\section{1,9-Bis(2,4-dinitrobenzenesulfenyl)-5-(4-methylphenyl)dipyrromethane}

GP5: 2-(2,4-dinitrobenzenesulfenyl)pyrrole $\quad(3) \quad(0.289 \quad \mathrm{~g}, \quad 1.1 \mathrm{mmol}) ; \quad 4-$ methylbenzaldehyde (0.05 mL, $0.42 \mathrm{mmol})$; TFA (0.1 mL, $1.3 \mathrm{mmol}) ; \mathrm{CH}_{2} \mathrm{Cl}_{2}(25 \mathrm{~mL})$; $20 \mathrm{~h}$; column chromatography $\left(\mathrm{SiO}_{2}, 3 \times 5 \mathrm{~cm}\right.$, hexanes: $\mathrm{CH}_{2} \mathrm{Cl}_{2}$ 3:7) gave the title compound (0.135 g, 51\%), as a yellow-red solid. mp 134-137 ${ }^{\circ} \mathrm{C} ;{ }^{1} \mathrm{H}$ NMR (DMSO-d6) $\delta$ $10.62(\mathrm{~s}, 2 \mathrm{H}, \mathrm{NH}), 8.89[\mathrm{~d}, J=2.45,2 \mathrm{H}, 19(25)-\mathrm{H}], 8.18[\mathrm{dd}, J=2.5, J=9.05,2 \mathrm{H}$, 21(27)-H], 7.21 [AB system, $J=8.2,2 \mathrm{H}, 12(15)-\mathrm{H}$ or $11(16)-\mathrm{H}], 7.18$ [AB system., $J=$ $8.1,2 \mathrm{H}, 12(15)-\mathrm{H}$ or 11(16)-H], $7.11[\mathrm{~d}, J=9.0,2 \mathrm{H}, 22(28)-\mathrm{H}], 6.59[\mathrm{dd}, J=2.6, J=$ $3.35,2 \mathrm{H}, 2(8)-\mathrm{H}$ or $3(7)-\mathrm{H}], 6.10[\mathrm{dd}, J=2.8=2.8,2 \mathrm{H}, 2(8)-\mathrm{H}$ or $3(7)-\mathrm{H}], 5.65(\mathrm{~s}, 1 \mathrm{H}$, H-5), 2.14 (s, 3H, H-14); ${ }^{13} \mathrm{C}$ NMR (DMSO-d6) $\delta 149.9,145.5,144.5,140.6,139.2$, $137.4,130.1,129.9,129.1,127.8,121.6,121.1,112.4,111.3,44.8(\mathrm{CH}, \mathrm{C}-5), 21.0\left(\mathrm{CH}_{3}\right.$, 
Comparison of benzene, nitrobenzene and dinitrobenzene 2-arylsulfenylpyrroles

Jose R. Garabatos-Perera, Benjamin H. Rotstein and Alison Thompson*

C-14); $\mathrm{MS}(\mathrm{ESI}+) \mathrm{m} / \mathrm{z} 655.5\left(\mathrm{M}^{+}+\mathrm{Na}, 55\right), 1287.3\left(2 \mathrm{M}^{+}+\mathrm{Na}, 100\right) ;$ HRMS (ESI+, $\mathrm{C}_{28} \mathrm{H}_{20} \mathrm{~N}_{6} \mathrm{NaO}_{8} \mathrm{~S}_{2}$ ) calcd. 655.0676, found 655.0680.

\section{1,9-Bis(2,4-dinitrobenzenesulfenyl)-5-(4-nitrophenyl)dipyrromethane}

GP5: 2-(2,4-dinitrobenzenesulfenyl)pyrrole (3) (0.252 g, 0.95 mmol); 4nitrobenzaldehyde (0.065 g, $0.43 \mathrm{mmol})$; TFA (0.2 mL, $2.6 \mathrm{mmol}) ; \mathrm{CH}_{2} \mathrm{Cl}_{2}(25 \mathrm{~mL}) ; 48$ h; column chromatography $\left(\mathrm{SiO}_{2}, 3 \times 5 \mathrm{~cm}, \mathrm{CH}_{2} \mathrm{Cl}_{2}\right.$ :EtOAc 9:1) gave the title compound (0.187 g, $65 \%$ ), as a red solid. mp 160-161 ${ }^{\circ} \mathrm{C} ;{ }^{1} \mathrm{H}$ NMR (DMSO-d6) $\delta 11.7$ (s, 2H, NH), $8.80[\mathrm{~d}, J=2.45,2 \mathrm{H}, 18(24)-\mathrm{H}], 8.28[\mathrm{~d}, J=8.8,2 \mathrm{H}, 11(15)-\mathrm{H}$ or $12(14)-\mathrm{H}], 8.24$ [dd, $J$ $=2.45, J=9.0,2 \mathrm{H}, 20(26)-\mathrm{H}], 7.55[\mathrm{~d}, J=8.65,2 \mathrm{H}, 11(15)-\mathrm{H}$ or $12(14)-\mathrm{H}], 6.93[\mathrm{~d}, J=$ $9.05,2 \mathrm{H} .21(27)-\mathrm{H}], 6.58[\mathrm{dd}, J=2.6=2.6,2 \mathrm{H}, 2(8)-\mathrm{H}$ or $3(7)-\mathrm{H}], 6.04[\mathrm{dd}, J=2.65=$ 2.65, 2H, 2(8)-H or 3(7)-H], 5.76 (s, 1H, H-5); ${ }^{13} \mathrm{C}$ NMR (DMSO-d6) $\delta ~ 149.2,148.3$, $146.4,144.2,143.1,137.9,129.5,128.9,127.5,123.8,120.9,119.9,111.2,110.3,43.3$ $(\mathrm{CH}, \mathrm{C}-5) ; \mathrm{MS}(\mathrm{ESI}+) \mathrm{m} / z 686.3\left(\mathrm{M}^{+}+\mathrm{Na}, 45\right), 1349.0\left(2 \mathrm{M}^{+}+\mathrm{Na}, 100\right)$; HRMS (ESI+, $\mathrm{C}_{27} \mathrm{H}_{17} \mathrm{~N}_{7} \mathrm{NaO}_{10} \mathrm{~S}_{2}$ ) calcd. 686.0371, found 686.0323.

\section{1,9-Bis(2,4-dinitrobenzenesulfenyl)-5-(4-pyridyl)dipyrromethane}

GP5: 2-(2,4-dinitrobenzenesulfenyl)pyrrole (3) $\quad(0.252 \quad \mathrm{~g}, \quad 0.95 \quad \mathrm{mmol}) ; \quad 4-$ pyridinecarboxaldehyde (0.04 mL, $0.42 \mathrm{mmol})$; TFA $(0.2 \mathrm{~mL}, 2.6 \mathrm{mmol}) ; \mathrm{CH}_{2} \mathrm{Cl}_{2}(25$ $\mathrm{mL}) ; 48 \mathrm{~h}$; column chromatography $\left(\mathrm{SiO}_{2}, 3 \times 5 \mathrm{~cm}, \mathrm{CH}_{2} \mathrm{Cl}_{2}:\right.$ EtOAc 3:7) gave the title compound $(0.108 \mathrm{~g}, 41 \%)$, as a red liquid. ${ }^{1} \mathrm{H}$ NMR (DMSO-d6) $\delta 11.68(\mathrm{~s}, 2 \mathrm{H}), 8.80(\mathrm{~d}$, $J=2.55,2 \mathrm{H}), 8.58(\mathrm{~d}, J=6.5,2 \mathrm{H}), 8.22(\mathrm{dd}, J=2.5, J=9.05,2 \mathrm{H}), 7.28(\mathrm{~d}, J=6,2 \mathrm{H})$, $6.93(\mathrm{~d}, J=9.05,2 \mathrm{H}), 6.57(\mathrm{dd}, J=2.5, J=3.4,2 \mathrm{H}), 6.04(\mathrm{dd}, J=2.85=2.65,2 \mathrm{H}), 5.61$ $(\mathrm{s}, 1 \mathrm{H}) ;{ }^{13} \mathrm{C}$ NMR (DMSO-d6) $\delta 150.7,150.3,148.8,144.6,143.6,138.2,129.5,127.9$, 
Comparison of benzene, nitrobenzene and dinitrobenzene 2-arylsulfenylpyrroles

Jose R. Garabatos-Perera, Benjamin H. Rotstein and Alison Thompson*

123.9, 121.4, 120.4, 111.6, 110.8, 110.3, 43.5; MS(ESI+) $m / z, 620.2\left(\mathrm{M}^{+}+\mathrm{H}, 100\right), 1260.6$

(2M+N $+\mathrm{Na}, 100)$. ); HRMS (ESI+, $\mathrm{C}_{28} \mathrm{H}_{18} \mathrm{~N}_{7} \mathrm{O}_{8} \mathrm{~S}_{2}$ ) calcd. 620.0653, found 620.0663.

\section{1,9-Bis(2,4-dinitrobenzenesulfenyl)-5-(4-bromophenyl)dipyrromethane}

GP5: 2-(2,4-dinitrobenzenesulfenyl)pyrrole (3) $\quad(0.251 \quad \mathrm{~g}, \quad 0.94 \quad \mathrm{mmol}) ; \quad 4-$ bromobenzaldehyde (0.087 g, $0.47 \mathrm{mmol})$; TFA (0.1 mL, $1.3 \mathrm{mmol}) ; \mathrm{CH}_{2} \mathrm{Cl}_{2}(25 \mathrm{~mL}) ; 24$ h; column chromatography $\left(\mathrm{SiO}_{2}, 3 \times 5 \mathrm{~cm}, \mathrm{CH}_{2} \mathrm{Cl}_{2}\right.$ :hexanes 9:1) gave the title compound (0.171 g, $0.24 \mathrm{mmol}, 52 \%)$, as a red solid. mp 138-141 ${ }^{\circ} \mathrm{C} ;{ }^{1} \mathrm{H}$ NMR (DMSO-d6) $\delta 11.60$ $(\mathrm{s}, 2 \mathrm{H}), 8.79(\mathrm{~d}, J=2.45,2 \mathrm{H}), 8.21(\mathrm{dd}, J=2.5, J=9.0,2 \mathrm{H}), 7.58(\mathrm{~d}, J=8.4,2 \mathrm{H}), 7.23$ $(\mathrm{d}, J=8.45,2 \mathrm{H}), 6.92(\mathrm{~d}, J=9.0,2 \mathrm{H}), 6.55(\mathrm{dd}, J=3.2, J=3.2,2 \mathrm{H}), 6.00(\mathrm{dd}, J=2.8, J$ = 2.8), $5.56(\mathrm{~s}, 1 \mathrm{H}) ;{ }^{13} \mathrm{C}$ NMR (DMSO-d6) $\delta 148.5,144.1,143.1,141.0,138.8,131.3$, 130.4, 129.0, 127.4, 120.9, 120.0, 119.9, 110.8, 110.1, 43.0; MS (ESI-) $m / z 696.9\left(\mathrm{M}^{+}-\mathrm{H}\right.$, 100). HRMS (ESI-, $\mathrm{C}_{27} \mathrm{H}_{16} \mathrm{BrN}_{6} \mathrm{O}_{8} \mathrm{~S}_{2}$ ) calcd. 694.9660, found 694.9653.

\section{Reductive desulfurization of 1,9-bis(2,4-dinitrobenzenesulfenyl)-5-}

\section{phenyldipyrromethane}

A suspension of 1,9-bis(2,4-dinitrobenzenesulfenyl)-5-phenyldipyrromethane $(0.025 \mathrm{~g}$, mmol) and Raney nickel ( 1.3 g, slurry in $5 \mathrm{~mL} \mathrm{EtOH})$ was heated to reflux for $2 \mathrm{~h}$, after which time TLC analysis showed the complete consumption of 1,9-bis $(2,4-$ dinitrosulfenyl)-5-phenyldipyrromethane. The reaction mixture was filtered and washed with EtOH. The filtrate was concentrated to dryness and the resulting residue was dissolved in $\mathrm{CH}_{2} \mathrm{Cl}_{2}$ and filtered through a silica pad $\left(\mathrm{CH}_{2} \mathrm{Cl}_{2}\right)$. The eluant was concentrated to afford 5-phenyldipyrromethane ${ }^{2}$ as a white solid (0.004 g, $\left.50 \%\right)$. 
Comparison of benzene, nitrobenzene and dinitrobenzene 2-arylsulfenylpyrroles Jose R. Garabatos-Perera, Benjamin H. Rotstein and Alison Thompson*

\section{NMR spectra}

${ }^{13} \mathrm{C}$ spectrum, 5-(4-Nitrobenzenesulfenyl)-pyrrole-2-carboxaldehyde

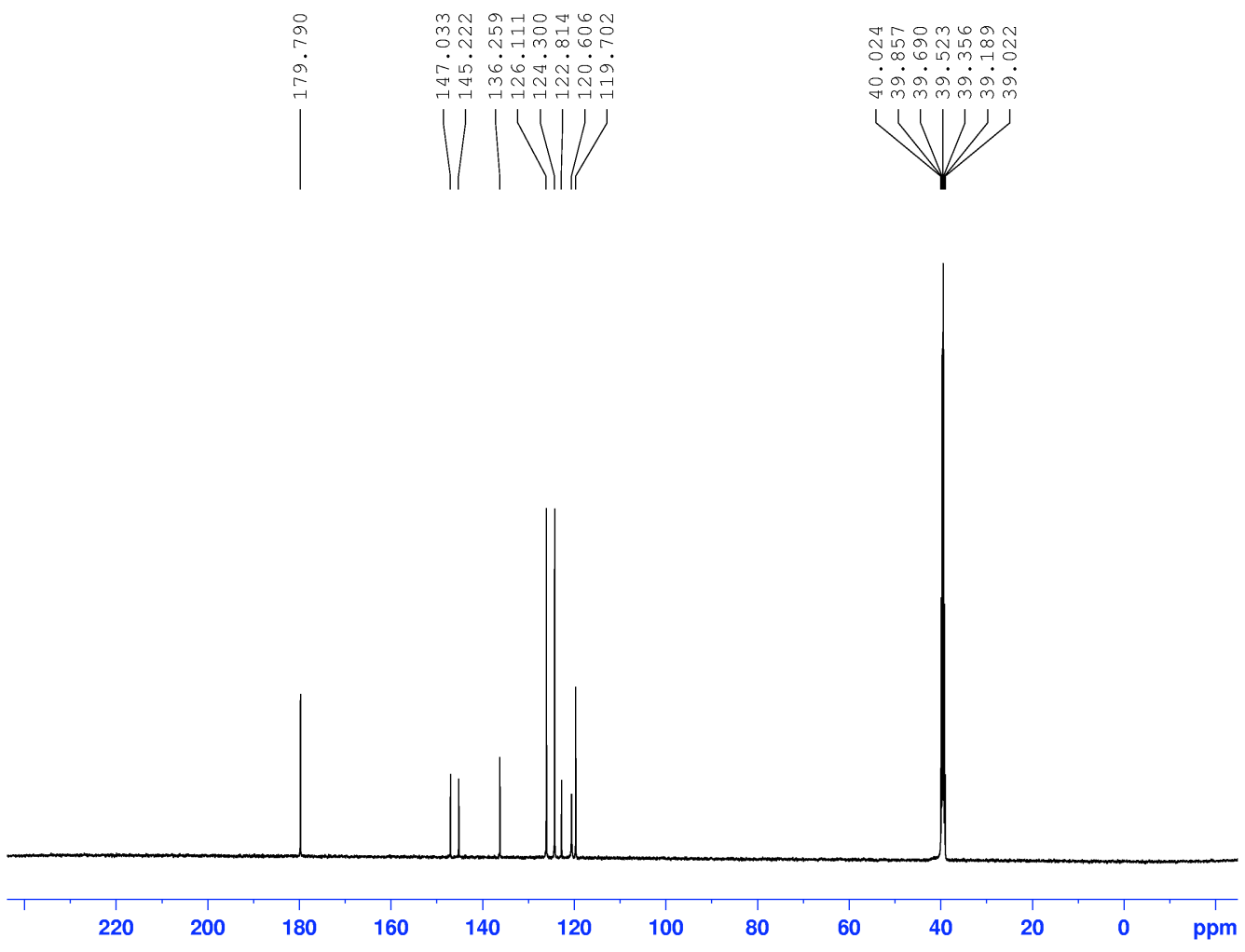


Comparison of benzene, nitrobenzene and dinitrobenzene 2-arylsulfenylpyrroles Jose R. Garabatos-Perera, Benjamin H. Rotstein and Alison Thompson*

\section{DEPTQ135 spectrum, 5-(4-Nitrobenzenesulfenyl)-pyrrole-2-carboxaldehyde}

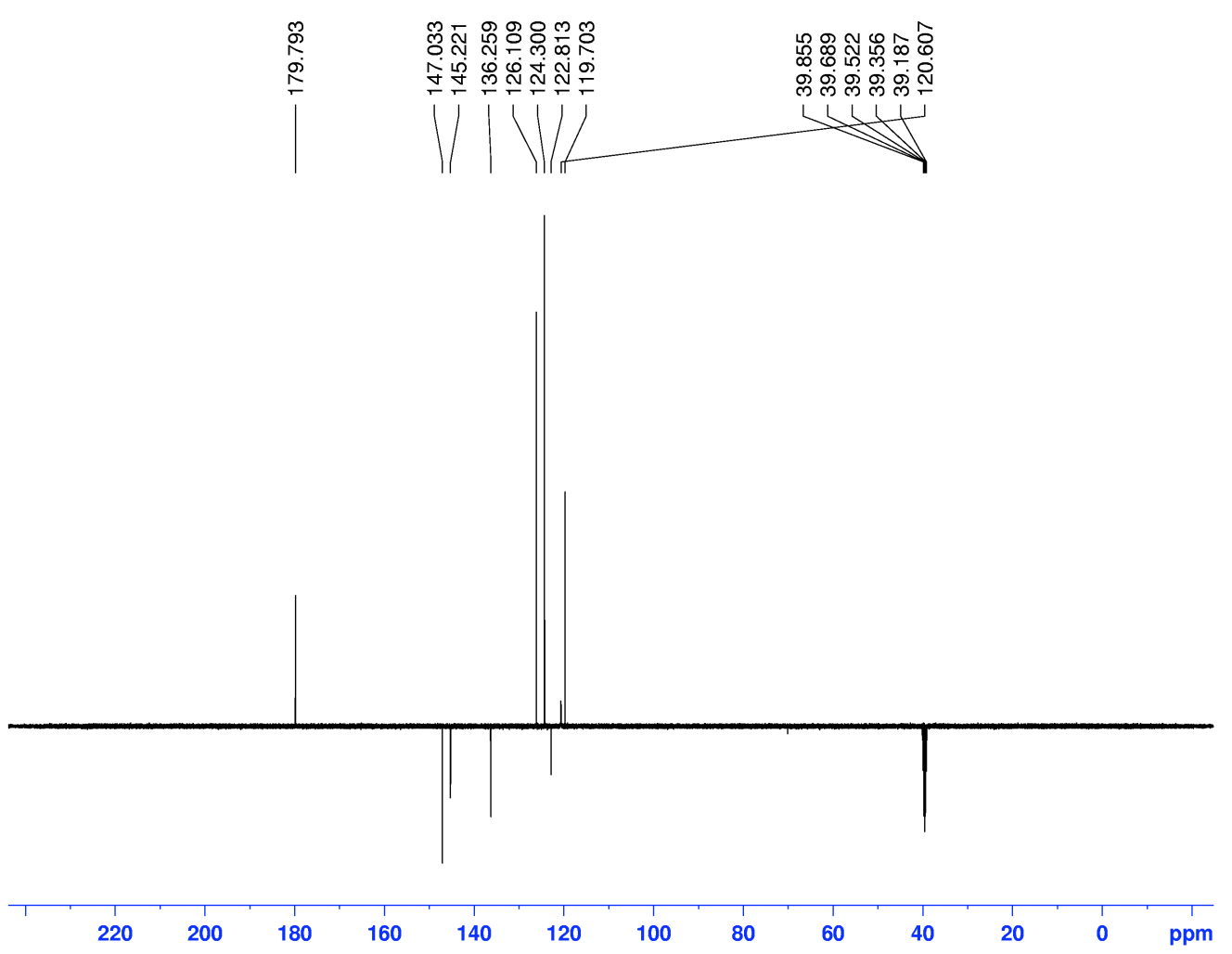


Comparison of benzene, nitrobenzene and dinitrobenzene 2-arylsulfenylpyrroles Jose R. Garabatos-Perera, Benjamin H. Rotstein and Alison Thompson*

\section{HMBC spectrum, 5-(4-Nitrobenzenesulfenyl)-pyrrole-2-carboxaldehyde}

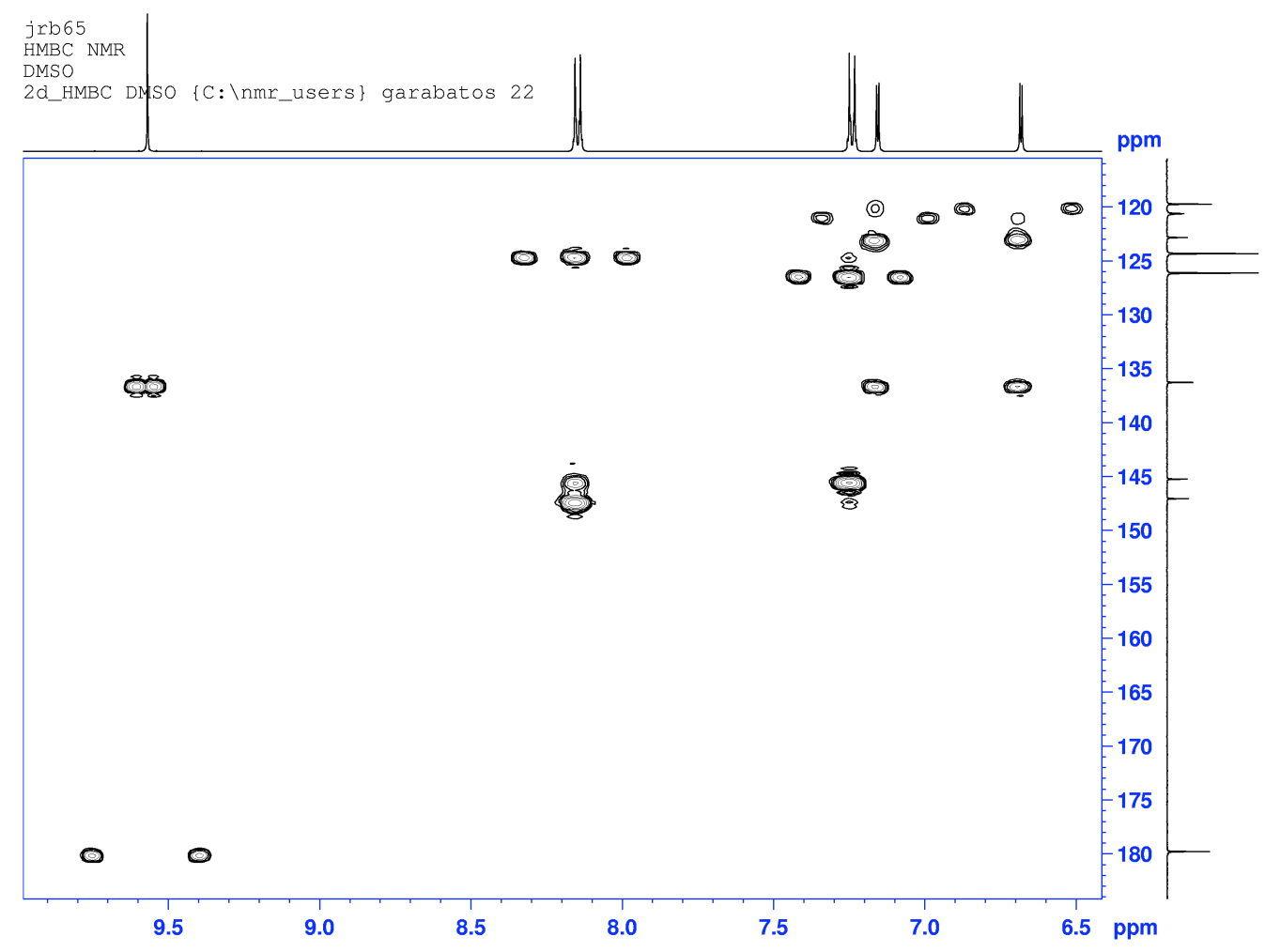


Comparison of benzene, nitrobenzene and dinitrobenzene 2-arylsulfenylpyrroles Jose R. Garabatos-Perera, Benjamin H. Rotstein and Alison Thompson*

\section{HMQC spectrum, 5-(4-Nitrobenzenesulfenyl)-pyrrole-2-carboxaldehyde}

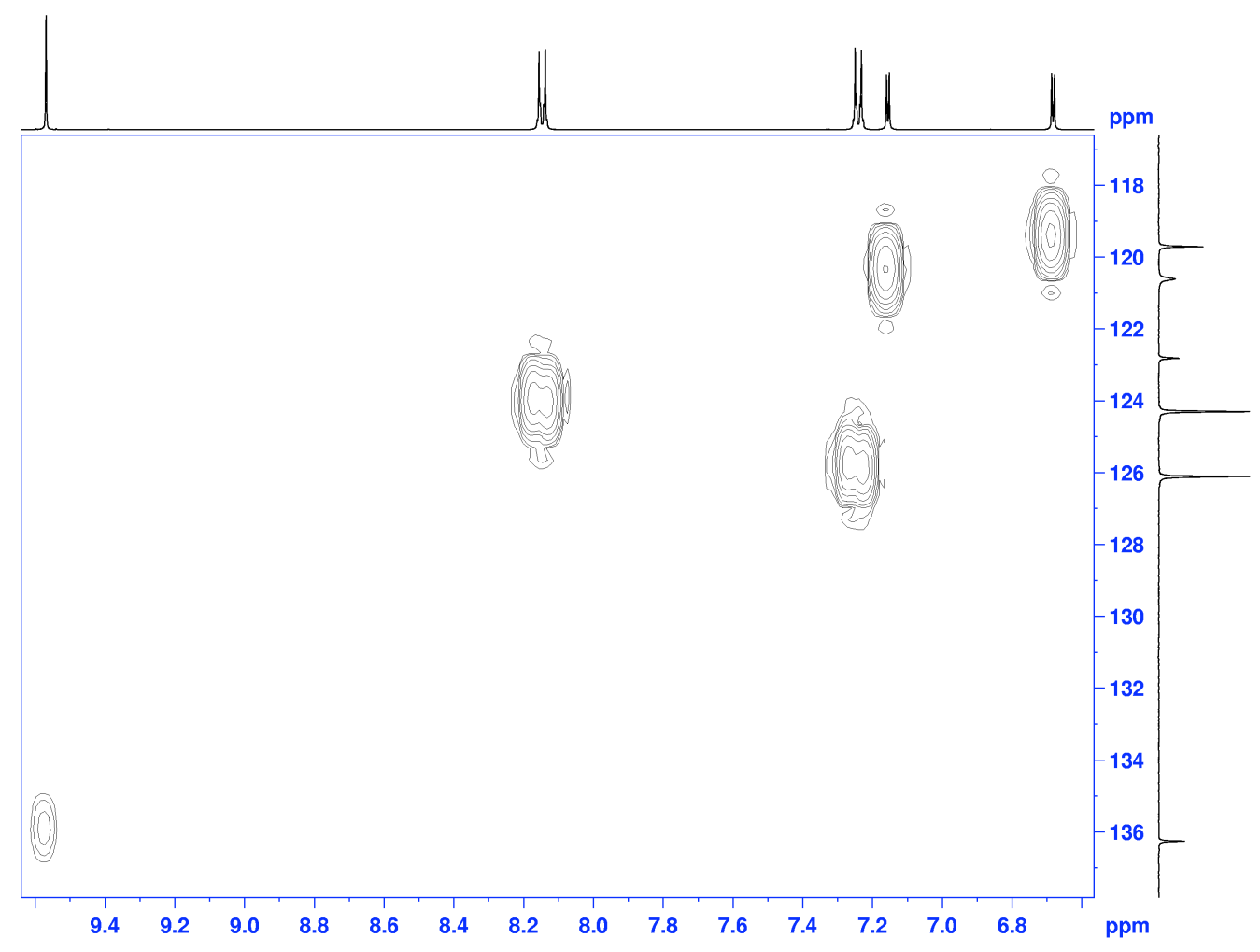


Comparison of benzene, nitrobenzene and dinitrobenzene 2 -arylsulfenylpyrroles Jose R. Garabatos-Perera, Benjamin H. Rotstein and Alison Thompson*

${ }^{13} \mathrm{C}$ spectrum, 5-(2,4-Dinitrobenzenesulfenyl)pyrrole-2-carboxaldehyde
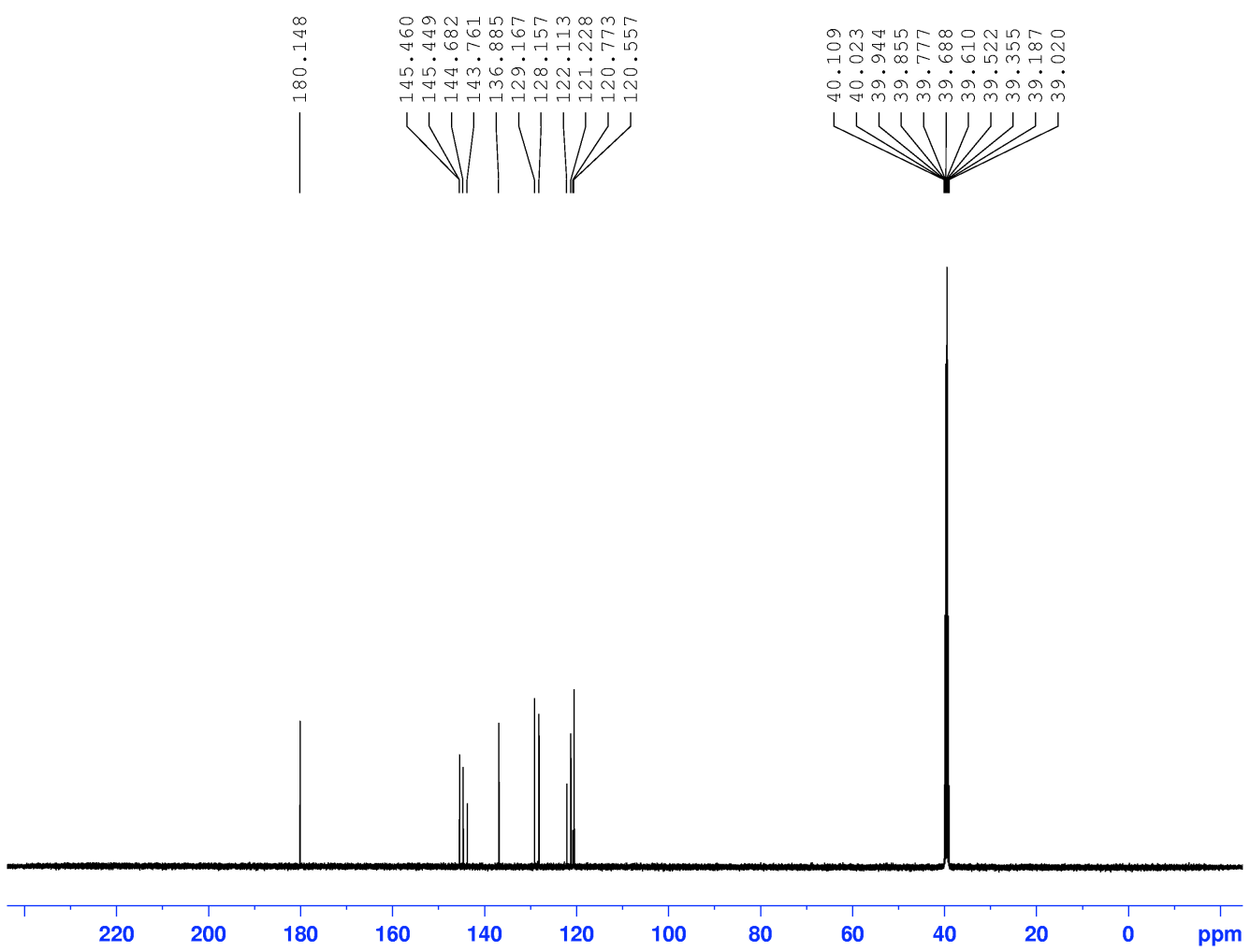
Comparison of benzene, nitrobenzene and dinitrobenzene 2-arylsulfenylpyrroles Jose R. Garabatos-Perera, Benjamin H. Rotstein and Alison Thompson*

\section{DEPTQ spectrum, 5-(2,4-Dinitrobenzenesulfenyl)pyrrole-2-carboxaldehyde}

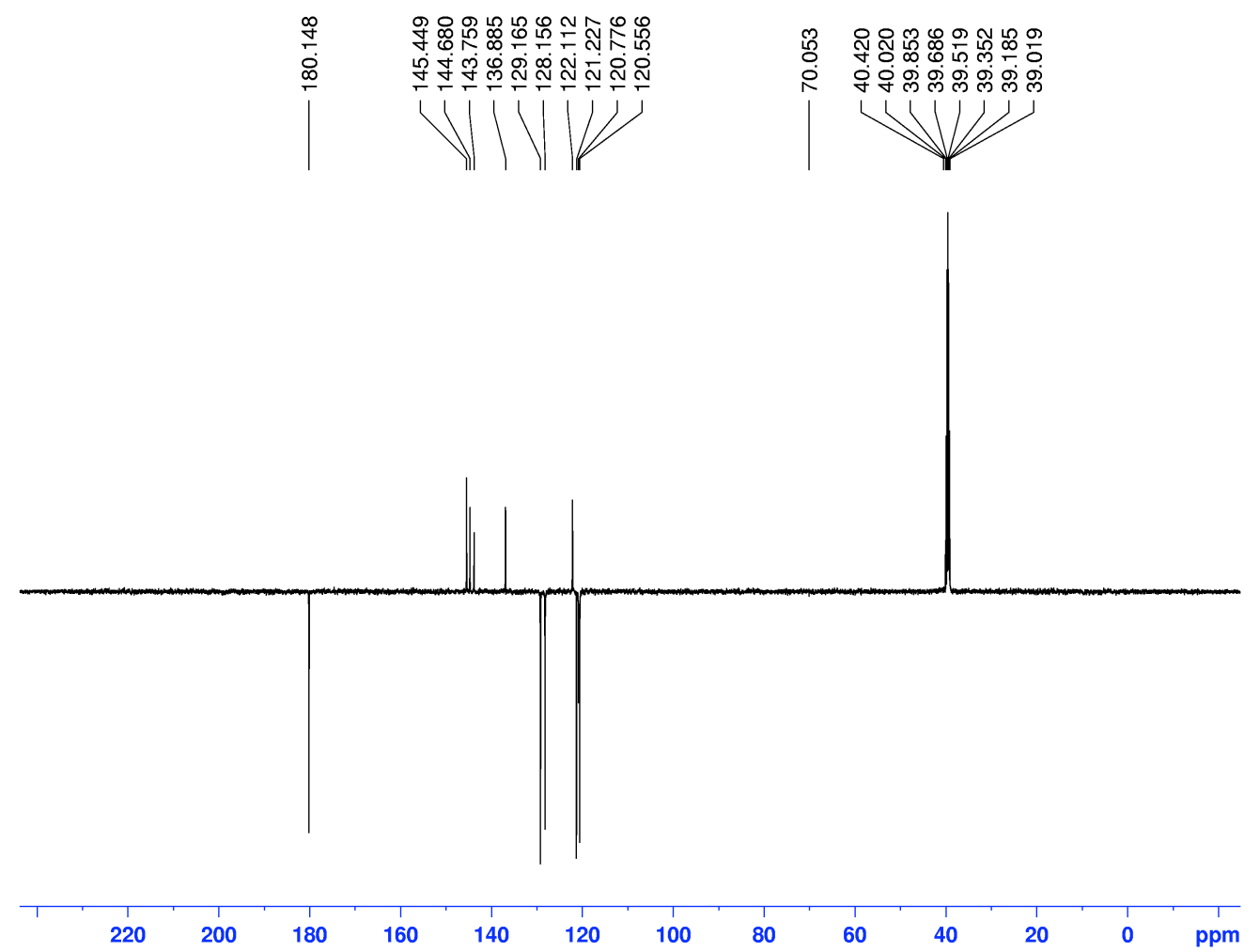


Comparison of benzene, nitrobenzene and dinitrobenzene 2-arylsulfenylpyrroles Jose R. Garabatos-Perera, Benjamin H. Rotstein and Alison Thompson*

\section{HMQC spectrum, 5-(2,4-Dinitrobenzenesulfenyl)pyrrole-2-carboxaldehyde}

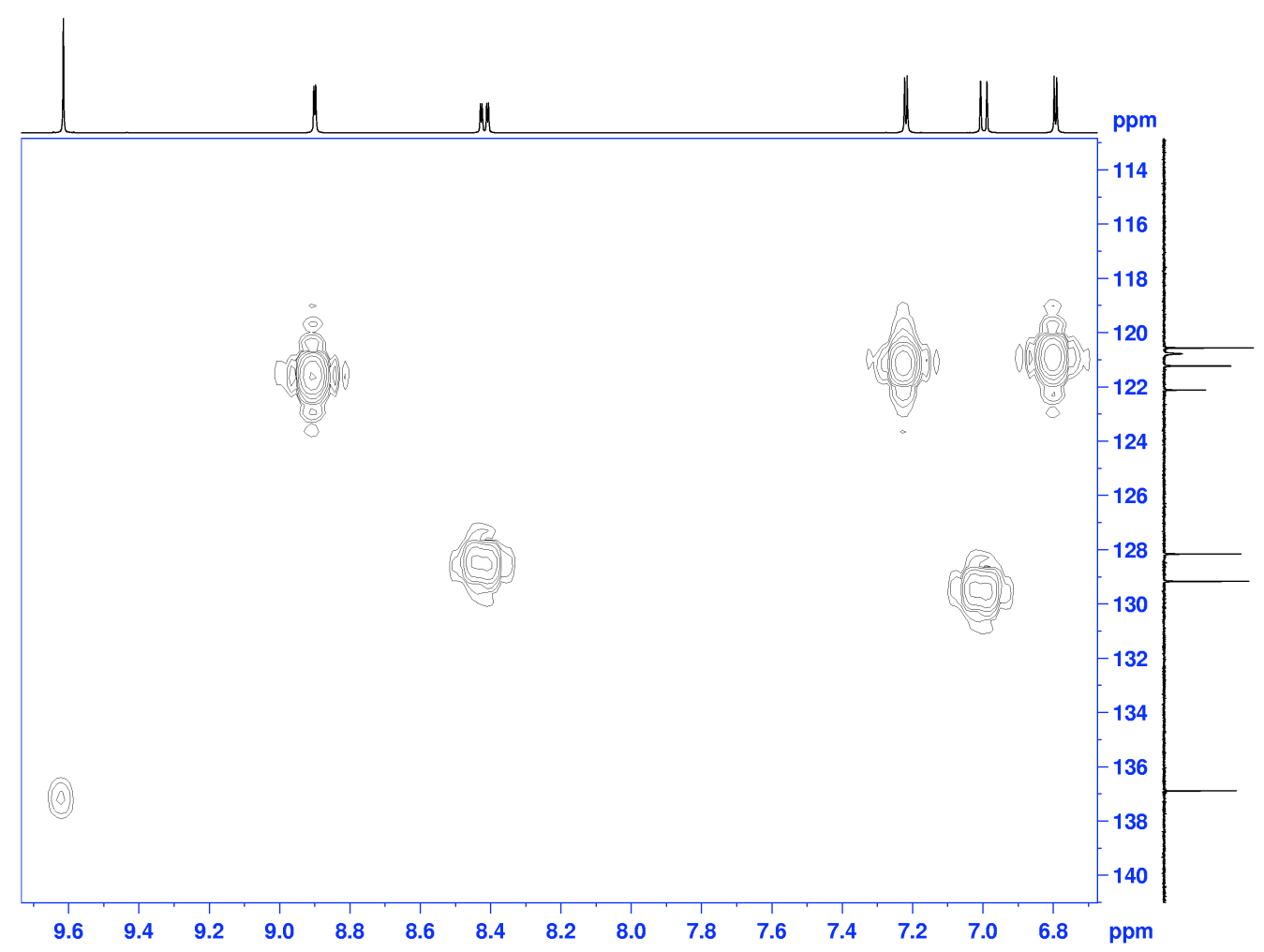


Comparison of benzene, nitrobenzene and dinitrobenzene 2-arylsulfenylpyrroles Jose R. Garabatos-Perera, Benjamin H. Rotstein and Alison Thompson*

HMBC, 5-(2,4-Dinitrobenzenesulfenyl)pyrrole-2-carboxaldehyde

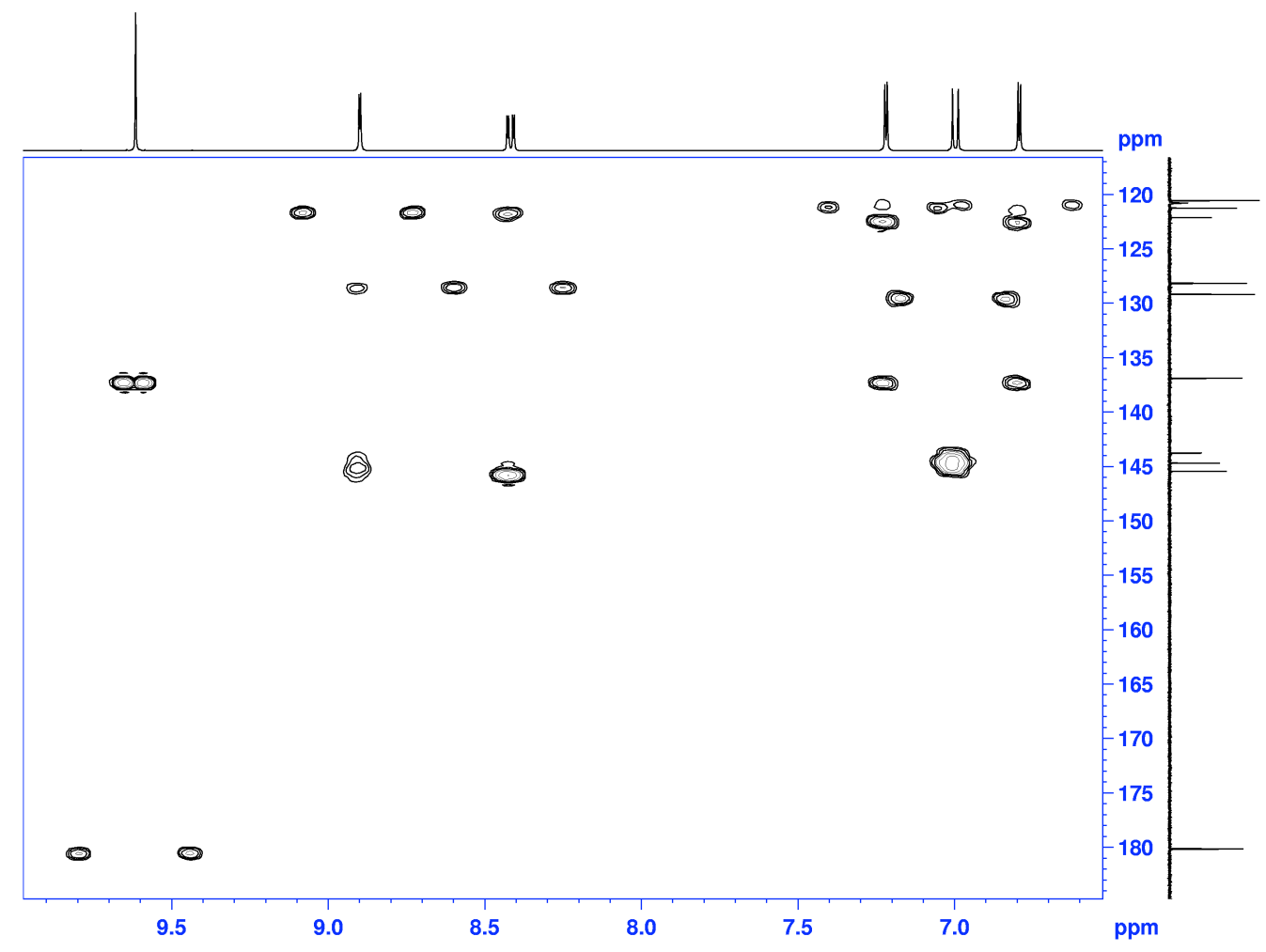


Comparison of benzene, nitrobenzene and dinitrobenzene 2-arylsulfenylpyrroles Jose R. Garabatos-Perera, Benjamin H. Rotstein and Alison Thompson*

${ }^{13} \mathrm{C}$ spectrum, 5-(2,4-Dinitrobenzenesulfenyl)pyrrole-3-carboxaldehyde

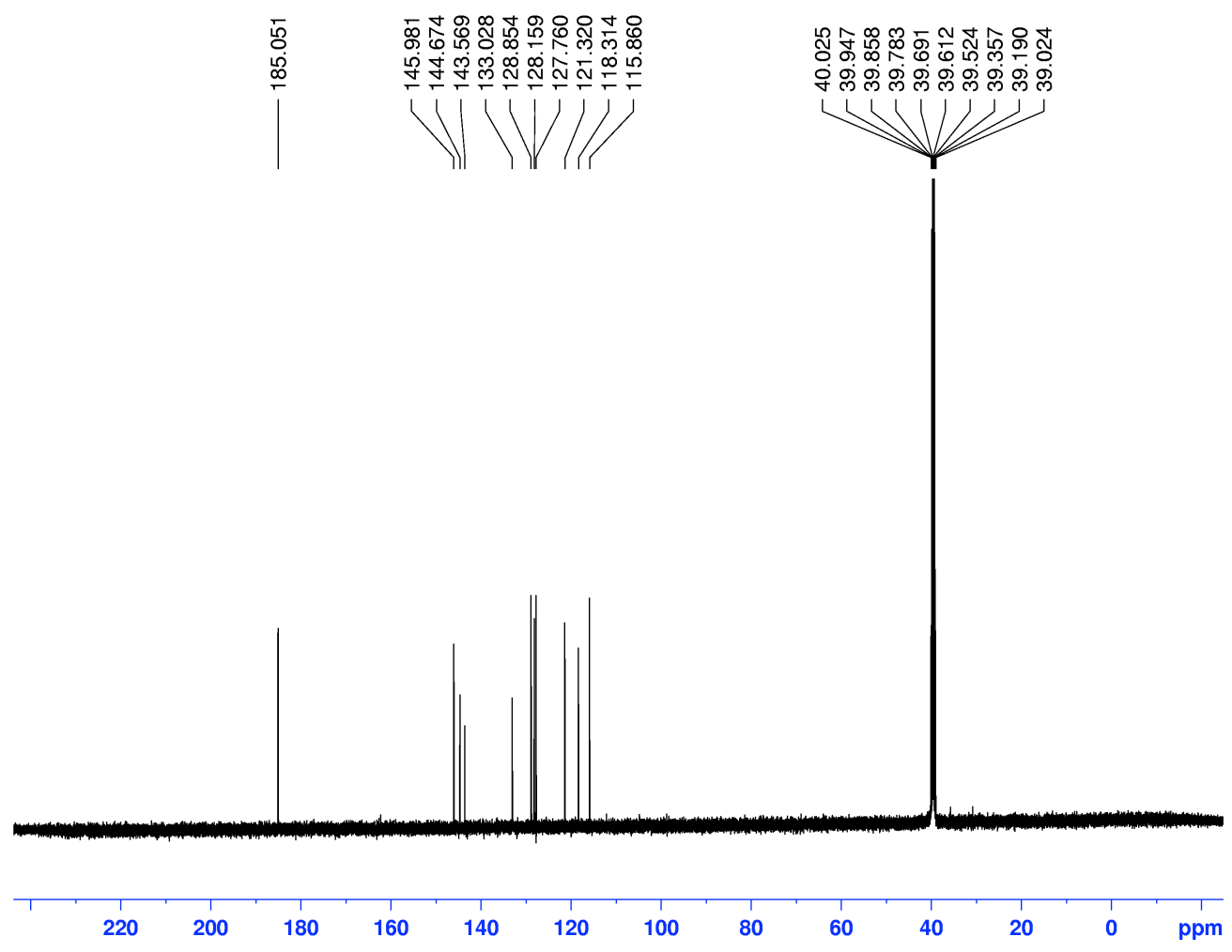


Comparison of benzene, nitrobenzene and dinitrobenzene 2-arylsulfenylpyrroles Jose R. Garabatos-Perera, Benjamin H. Rotstein and Alison Thompson*

\section{DEPTQ135 spectrum, 5-(2,4-Dinitrobenzenesulfenyl)pyrrole-3-carboxaldehyde}

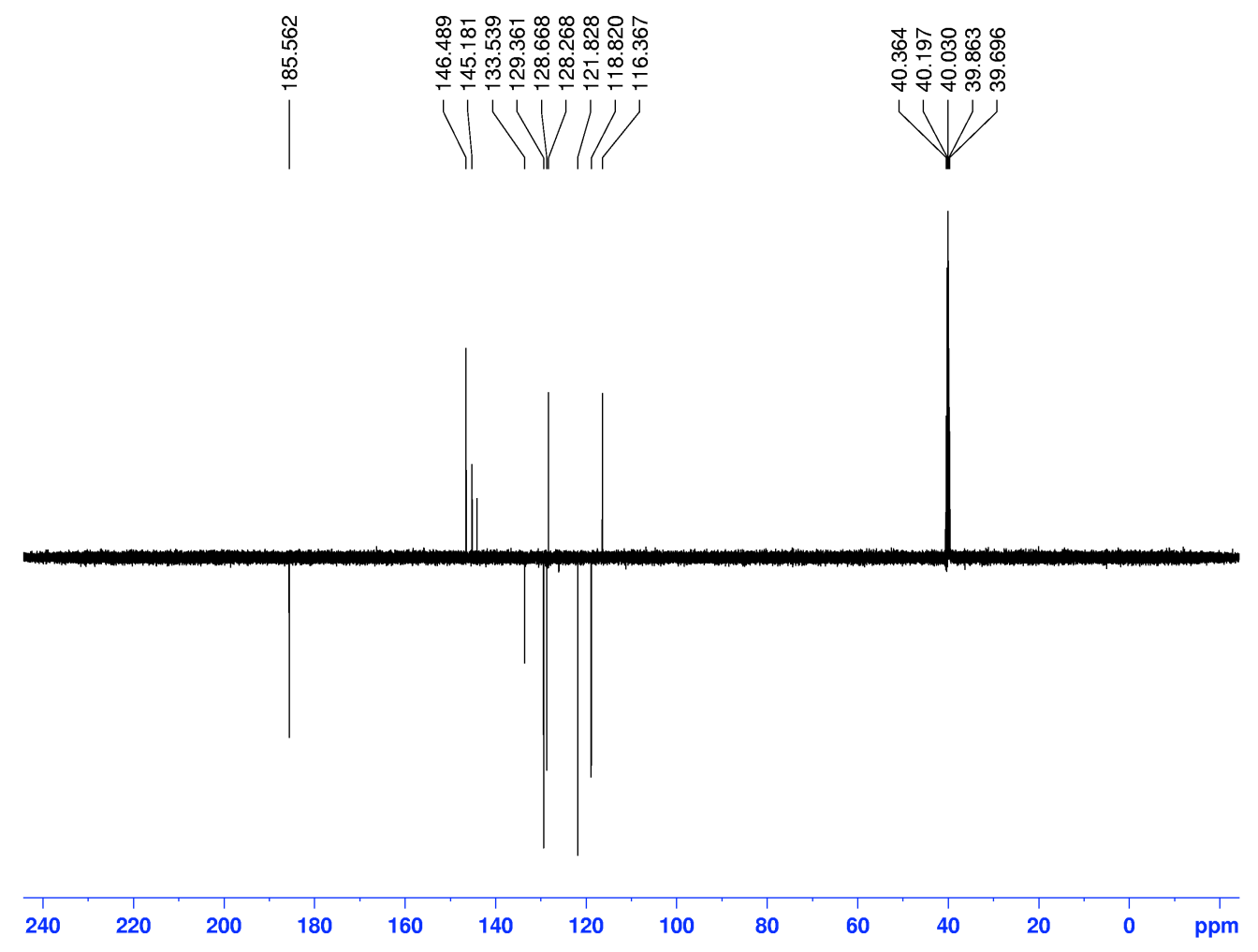


Comparison of benzene, nitrobenzene and dinitrobenzene 2-arylsulfenylpyrroles Jose R. Garabatos-Perera, Benjamin H. Rotstein and Alison Thompson*

\section{HMQC spectrum, 5-(2,4-Dinitrobenzenesulfenyl)pyrrole-3-carboxaldehyde}

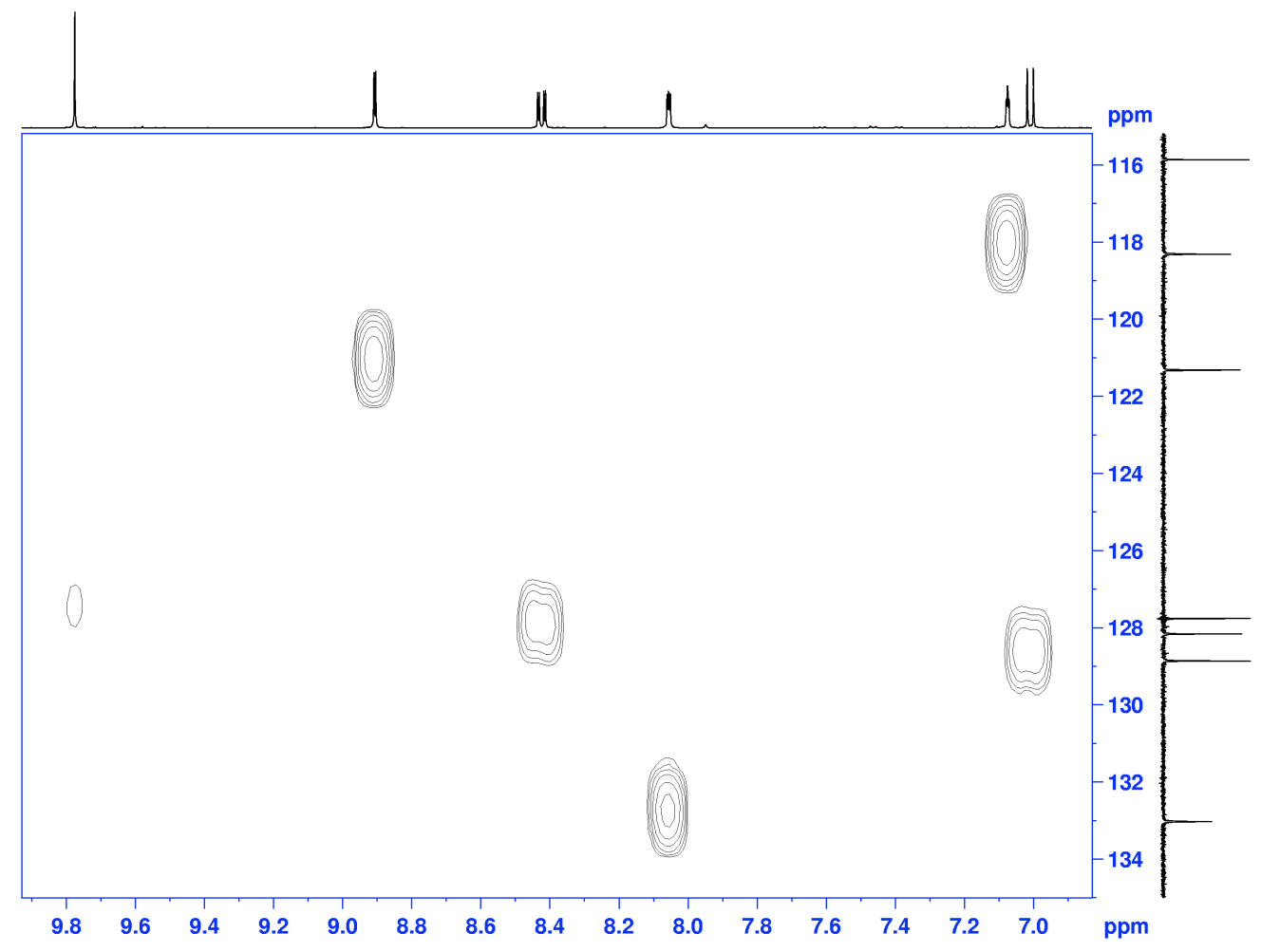


Comparison of benzene, nitrobenzene and dinitrobenzene 2-arylsulfenylpyrroles Jose R. Garabatos-Perera, Benjamin H. Rotstein and Alison Thompson*

\section{HMBC spectrum, 5-(2,4-Dinitrobenzenesulfenyl)pyrrole-3-carboxaldehyde}

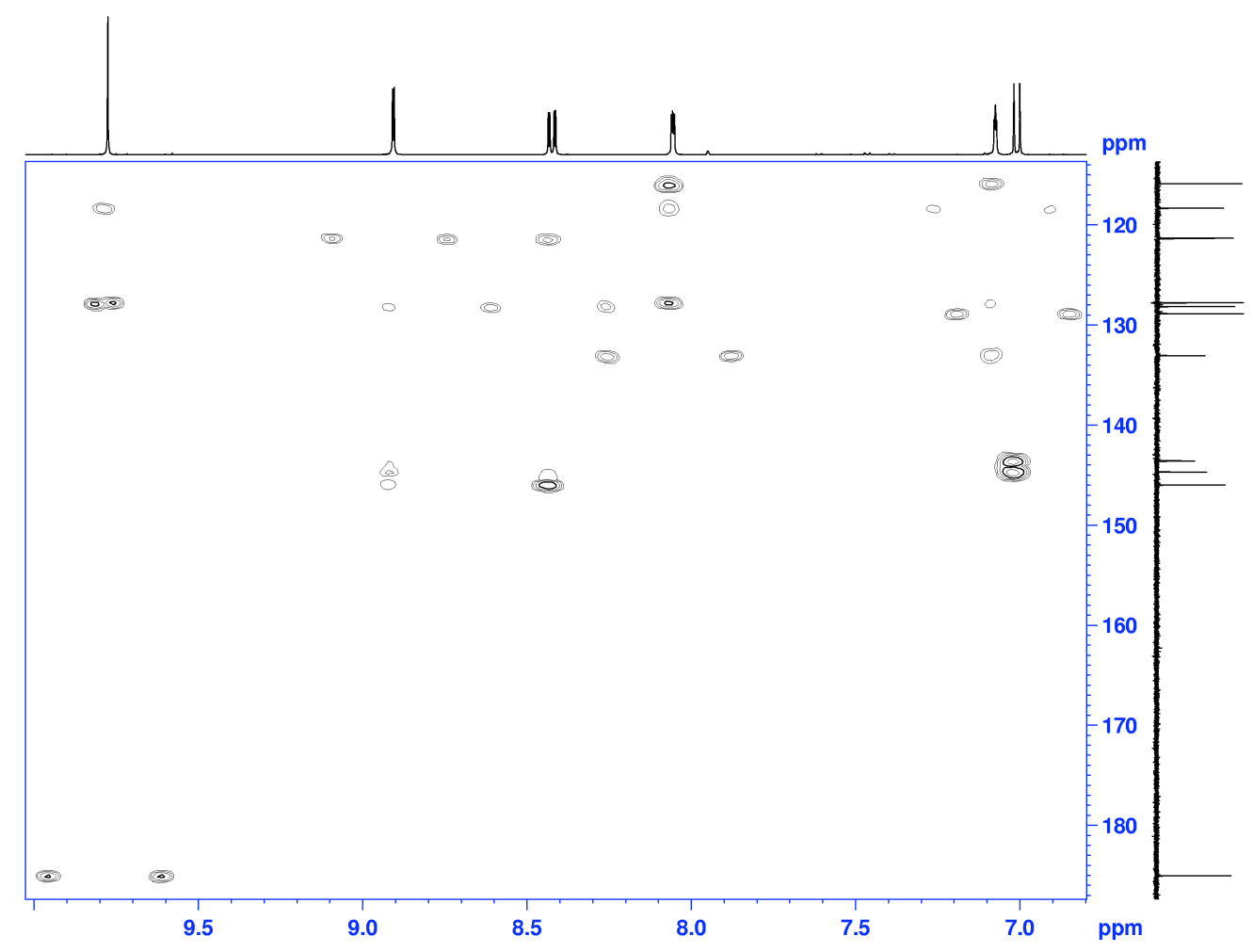


Comparison of benzene, nitrobenzene and dinitrobenzene 2-arylsulfenylpyrroles Jose R. Garabatos-Perera, Benjamin H. Rotstein and Alison Thompson*

NOESY spectrum, 5-(2,4-Dinitrobenzenesulfenyl)pyrrole-3-carboxaldehyde

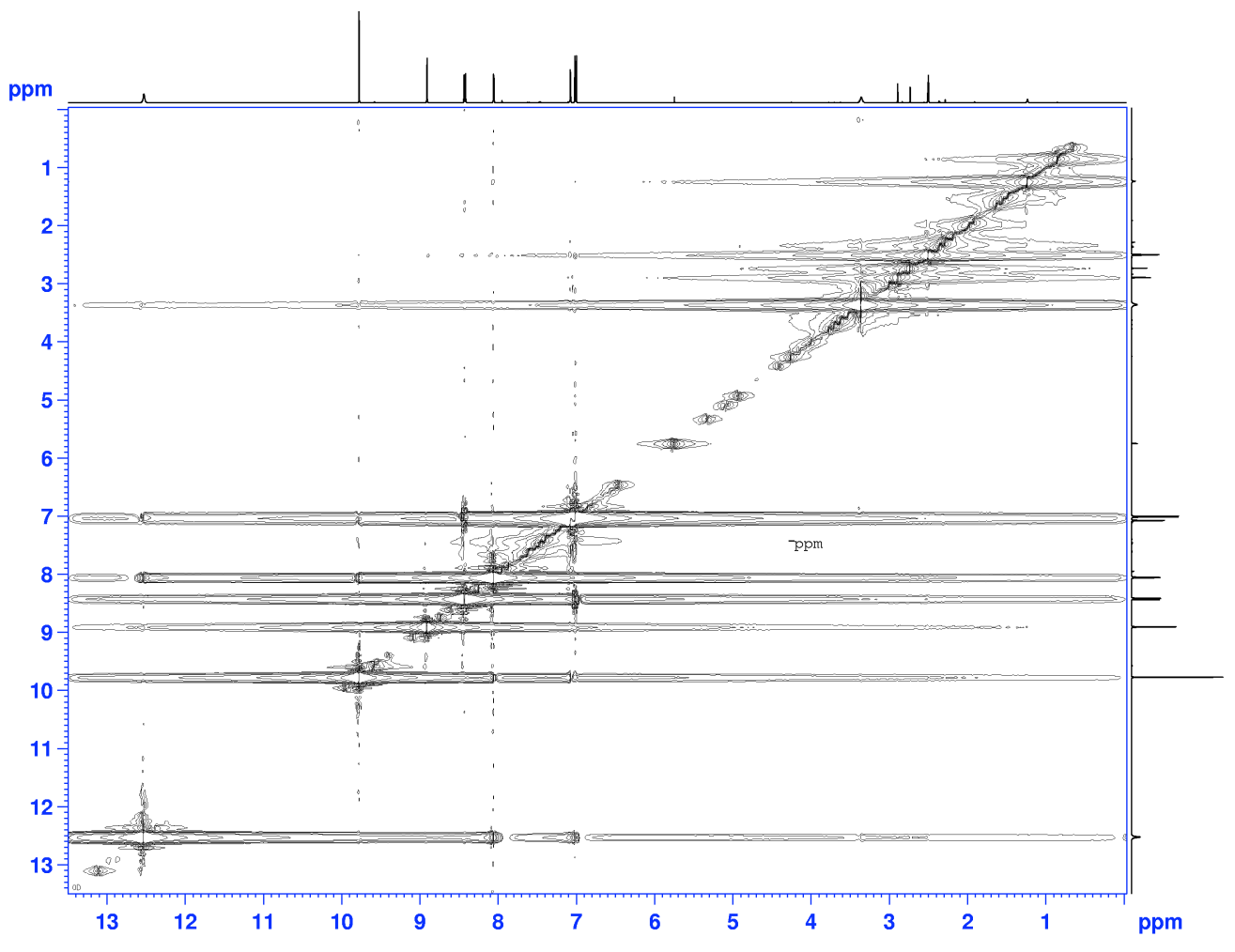


Comparison of benzene, nitrobenzene and dinitrobenzene 2 -arylsulfenylpyrroles Jose R. Garabatos-Perera, Benjamin H. Rotstein and Alison Thompson*

\section{${ }^{13} \mathrm{C}$ NMR spectrum, 2-Phenylthio-5-nitropyrrole}
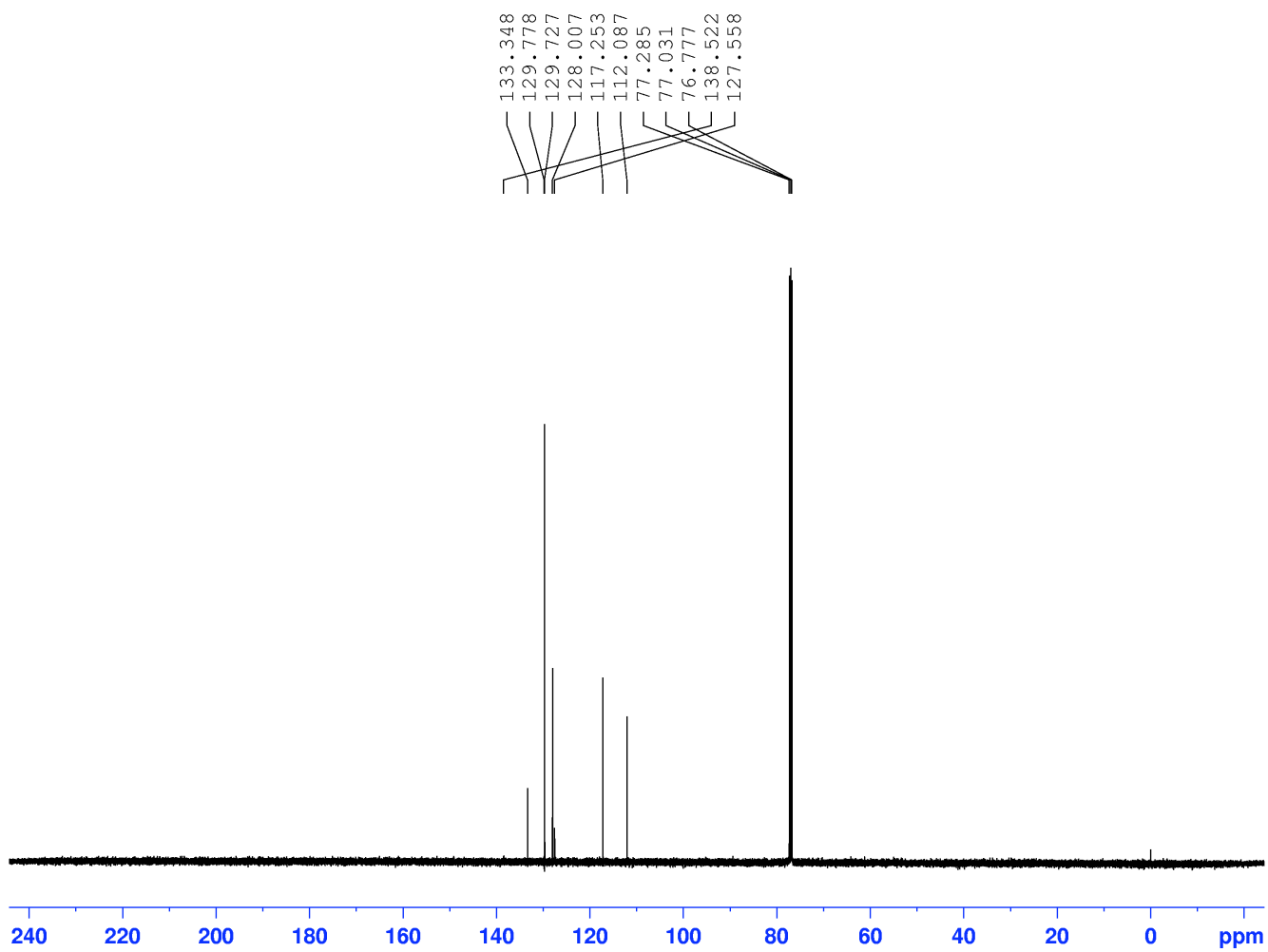
Comparison of benzene, nitrobenzene and dinitrobenzene 2-arylsulfenylpyrroles Jose R. Garabatos-Perera, Benjamin H. Rotstein and Alison Thompson*

\section{DEPTQ135 spectrum, 2-Phenylthio-5-nitropyrrole}

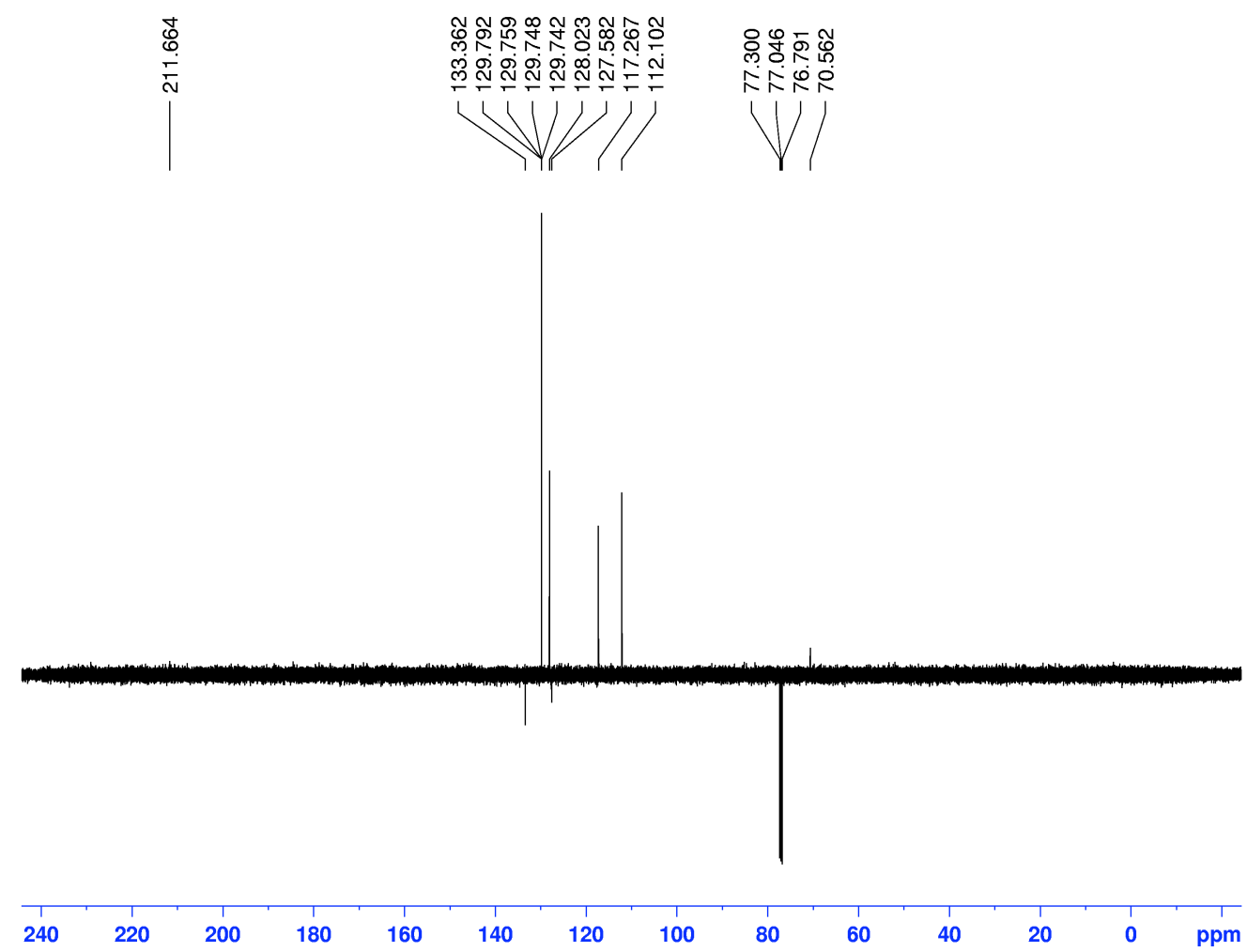


Comparison of benzene, nitrobenzene and dinitrobenzene 2-arylsulfenylpyrroles Jose R. Garabatos-Perera, Benjamin H. Rotstein and Alison Thompson*

\section{HMQC NMR spectrum, 2-Phenylthio-5-nitropyrrole}

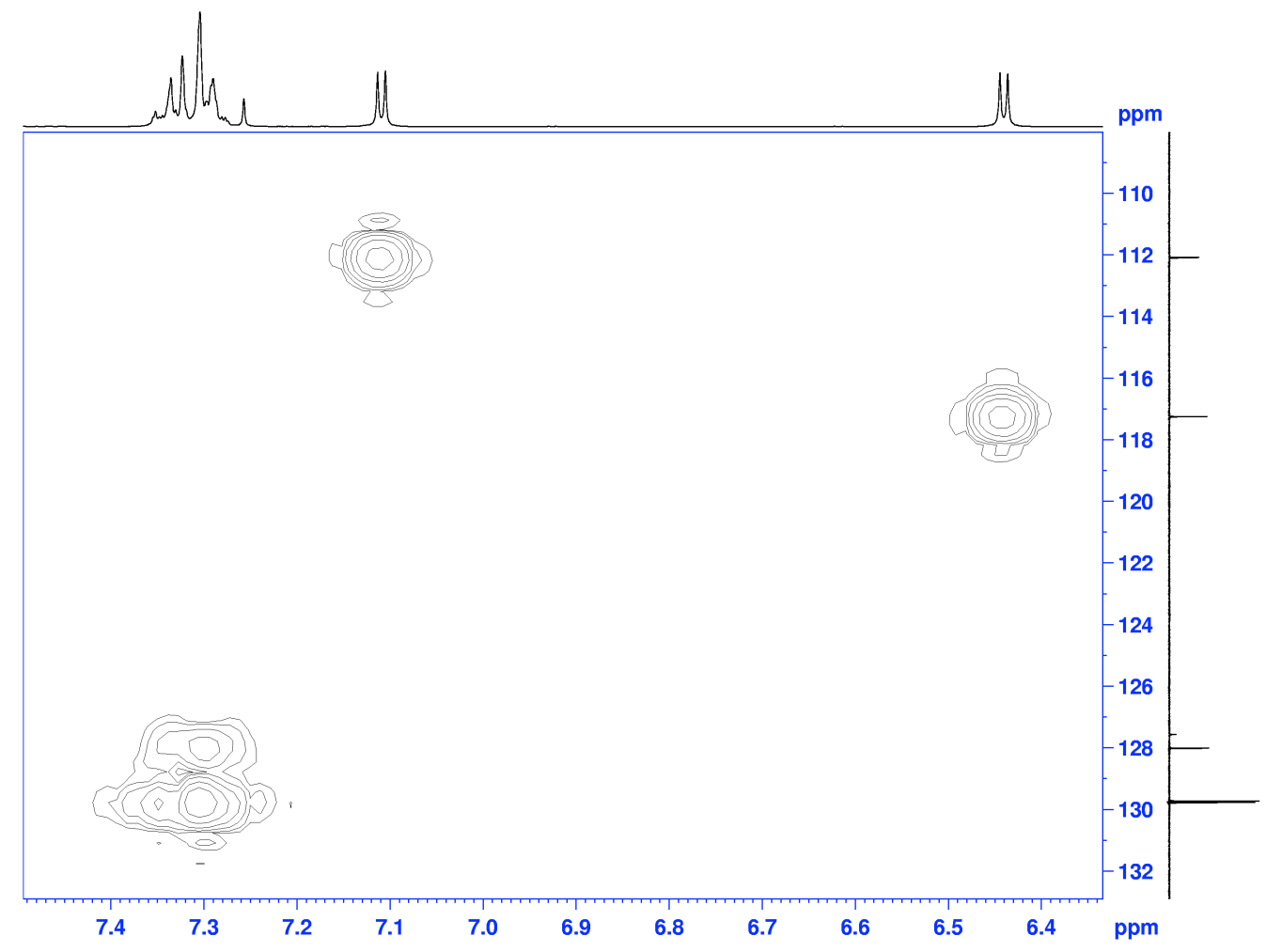


Comparison of benzene, nitrobenzene and dinitrobenzene 2-arylsulfenylpyrroles Jose R. Garabatos-Perera, Benjamin H. Rotstein and Alison Thompson*

\section{HMBC NMR spectrum, 2-Phenylthio-5-nitropyrrole}

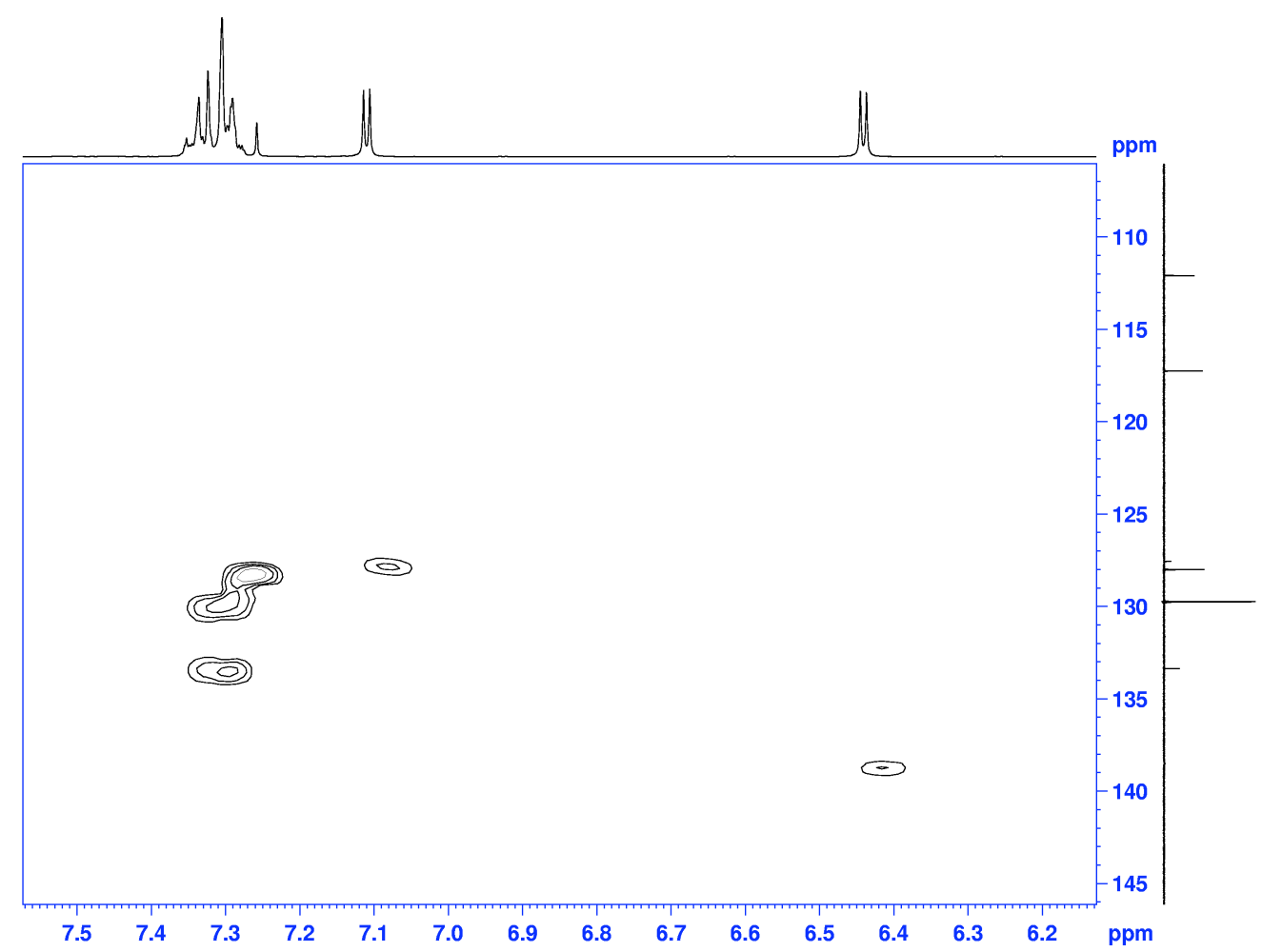


Comparison of benzene, nitrobenzene and dinitrobenzene 2 -arylsulfenylpyrroles Jose R. Garabatos-Perera, Benjamin H. Rotstein and Alison Thompson*

${ }^{13}$ C NMR spectrum, 2-Nitro-5-(4-nitrophenylsulfenyl)pyrrole
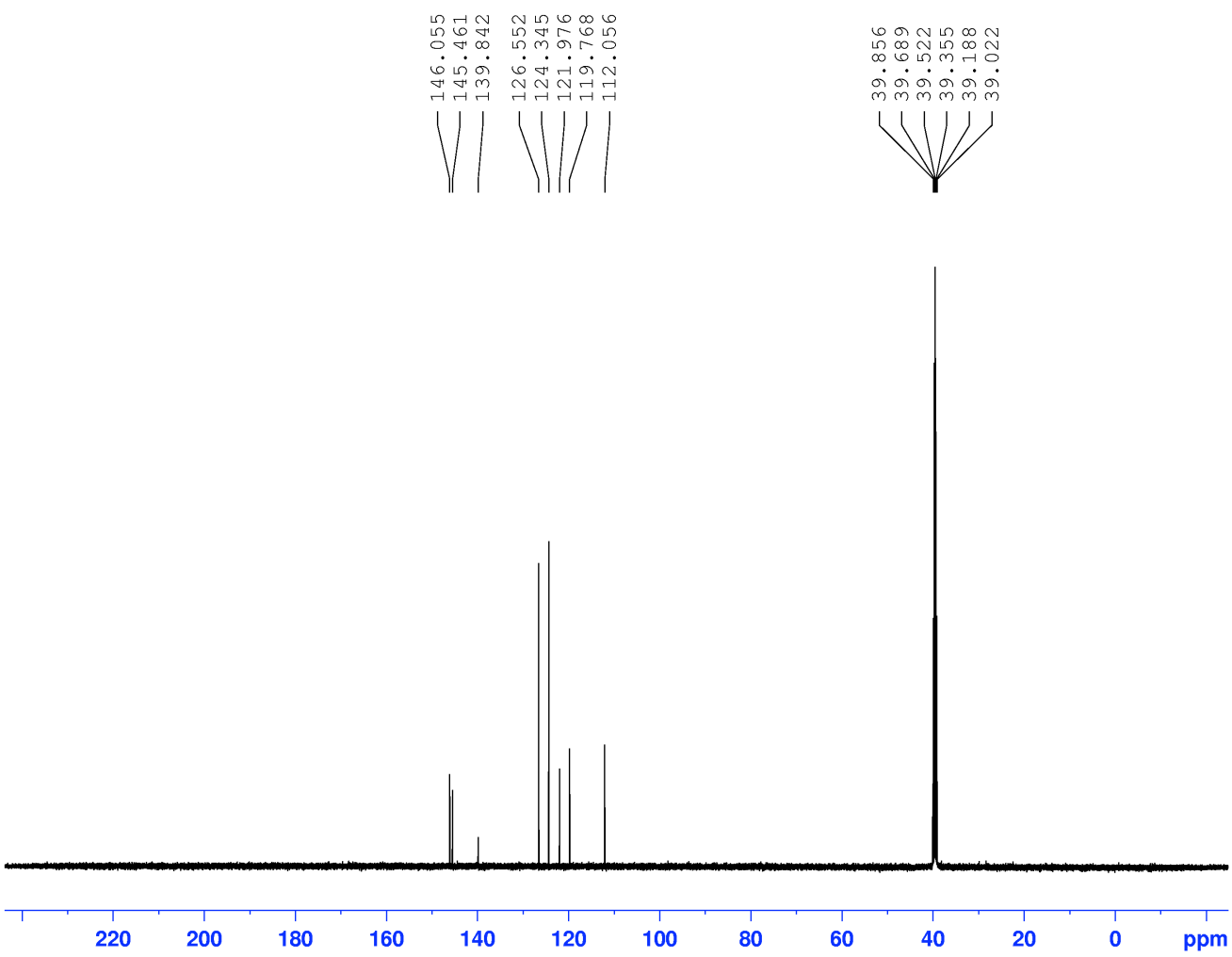
Comparison of benzene, nitrobenzene and dinitrobenzene 2 -arylsulfenylpyrroles Jose R. Garabatos-Perera, Benjamin H. Rotstein and Alison Thompson*

\section{DEPTQ135 NMR spectrum, 2-Nitro-5-(4-nitrophenylsulfenyl)pyrrole}

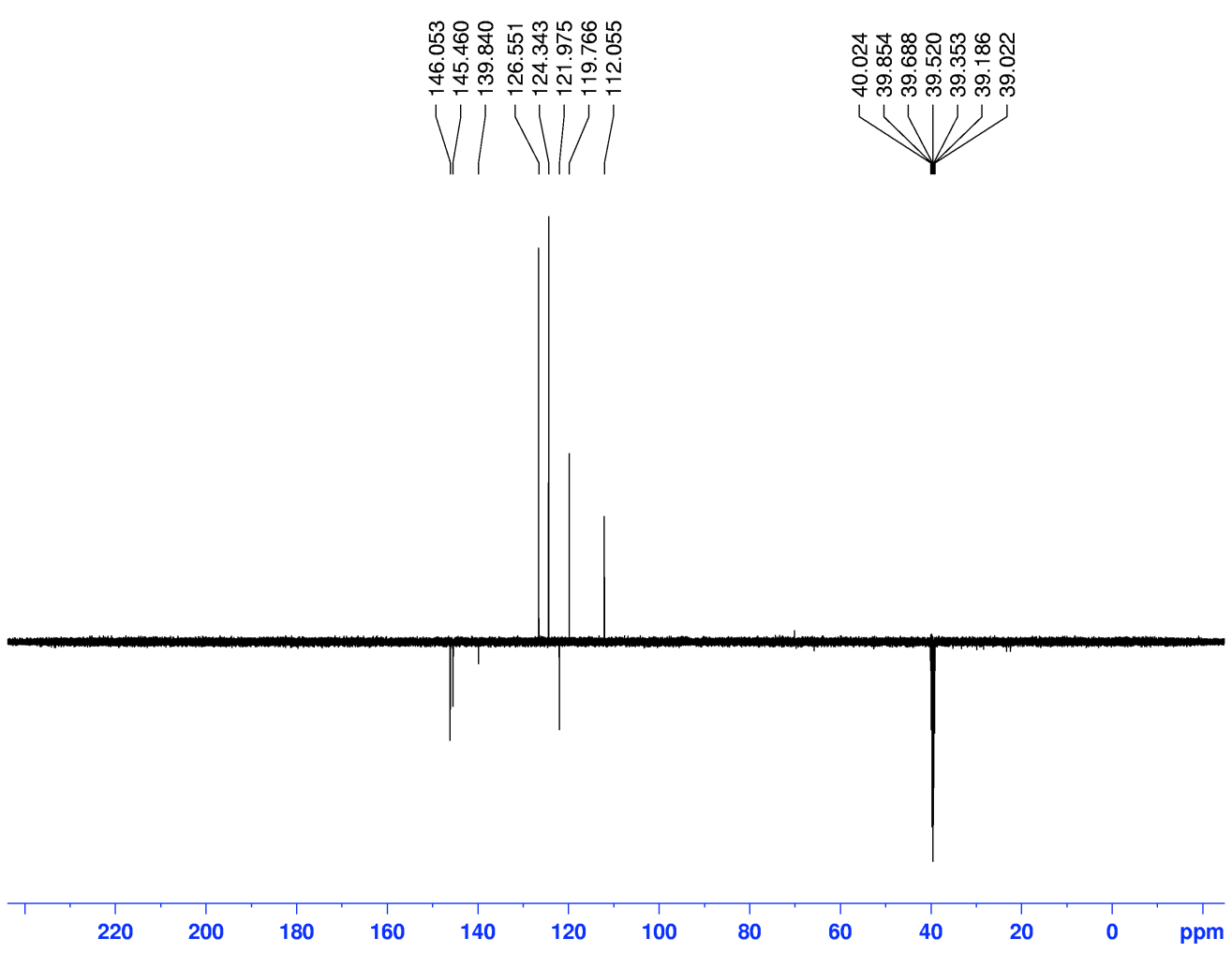


Comparison of benzene, nitrobenzene and dinitrobenzene 2-arylsulfenylpyrroles Jose R. Garabatos-Perera, Benjamin H. Rotstein and Alison Thompson*

\section{HMQC NMR spectrum, 2-Nitro-5-(4-nitrophenylsulfenyl)pyrrole}

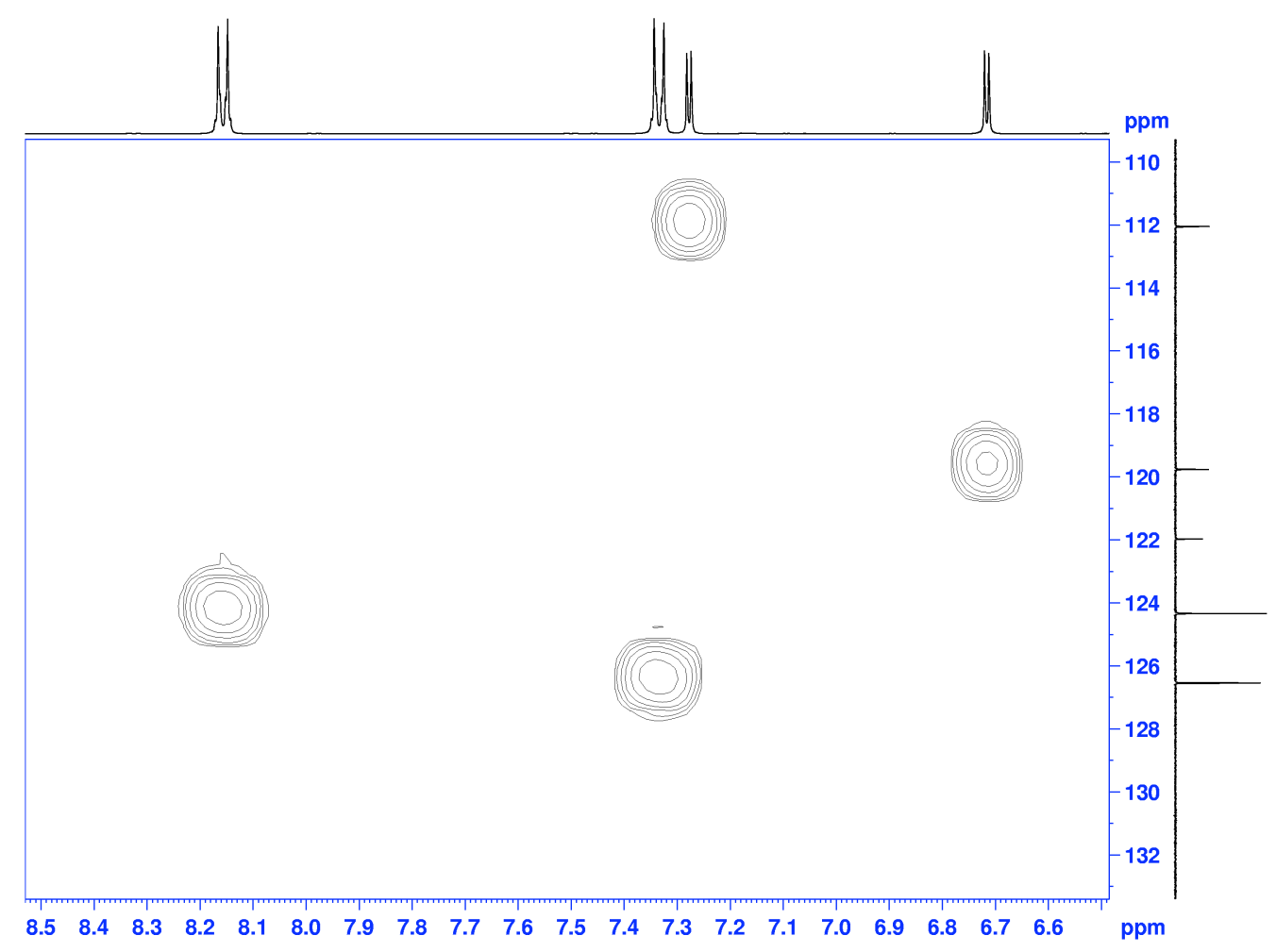


Comparison of benzene, nitrobenzene and dinitrobenzene 2-arylsulfenylpyrroles Jose R. Garabatos-Perera, Benjamin H. Rotstein and Alison Thompson*

\section{HMBC NMR spectrum, 2-Nitro-5-(4-nitrophenylsulfenyl)pyrrole}

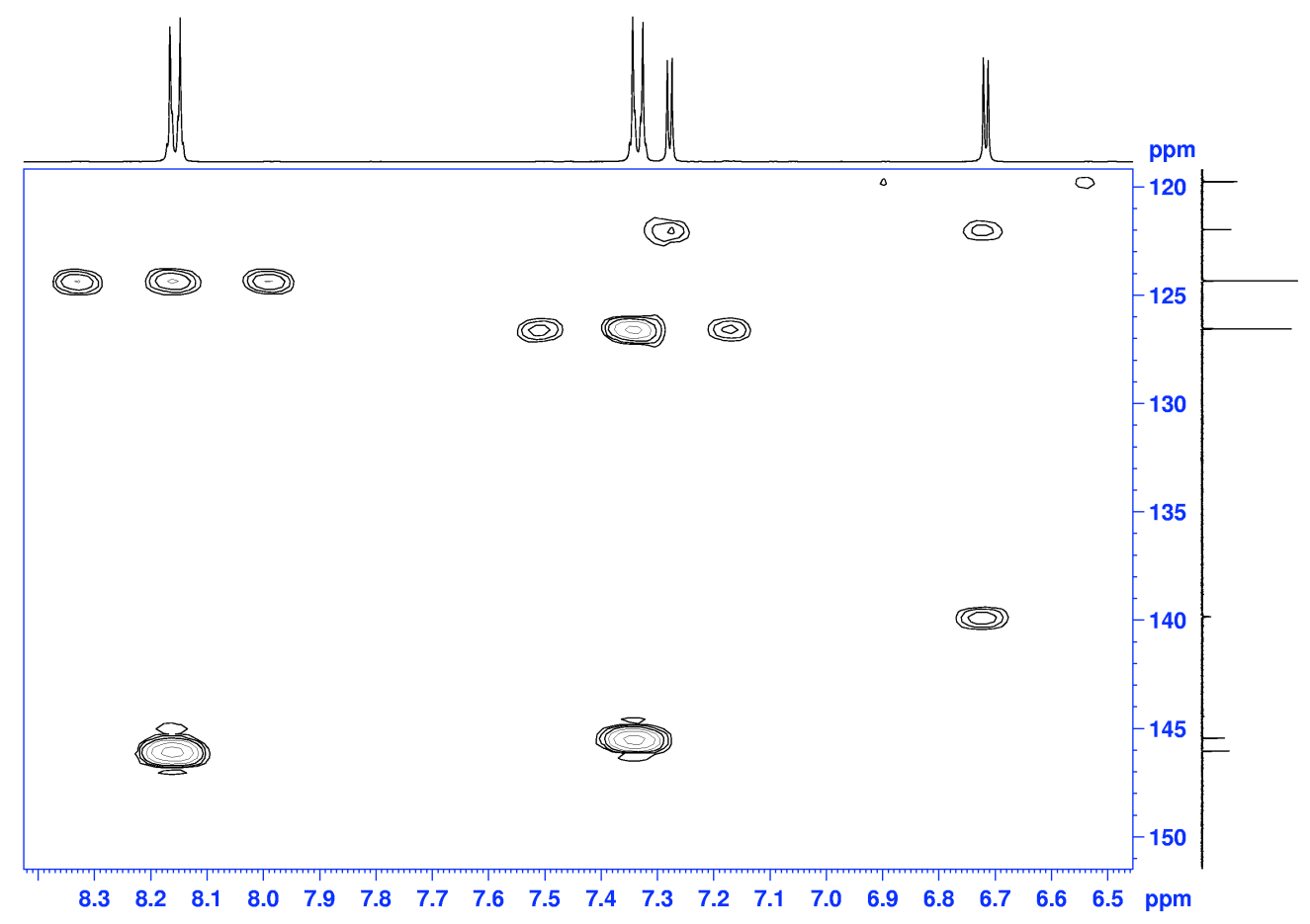


Comparison of benzene, nitrobenzene and dinitrobenzene 2 -arylsulfenylpyrroles Jose R. Garabatos-Perera, Benjamin H. Rotstein and Alison Thompson*

\section{${ }^{13}$ C NMR spectrum, 2-(2,4-Dinitrophenylsulfenyl)-5-nitropyrrole}
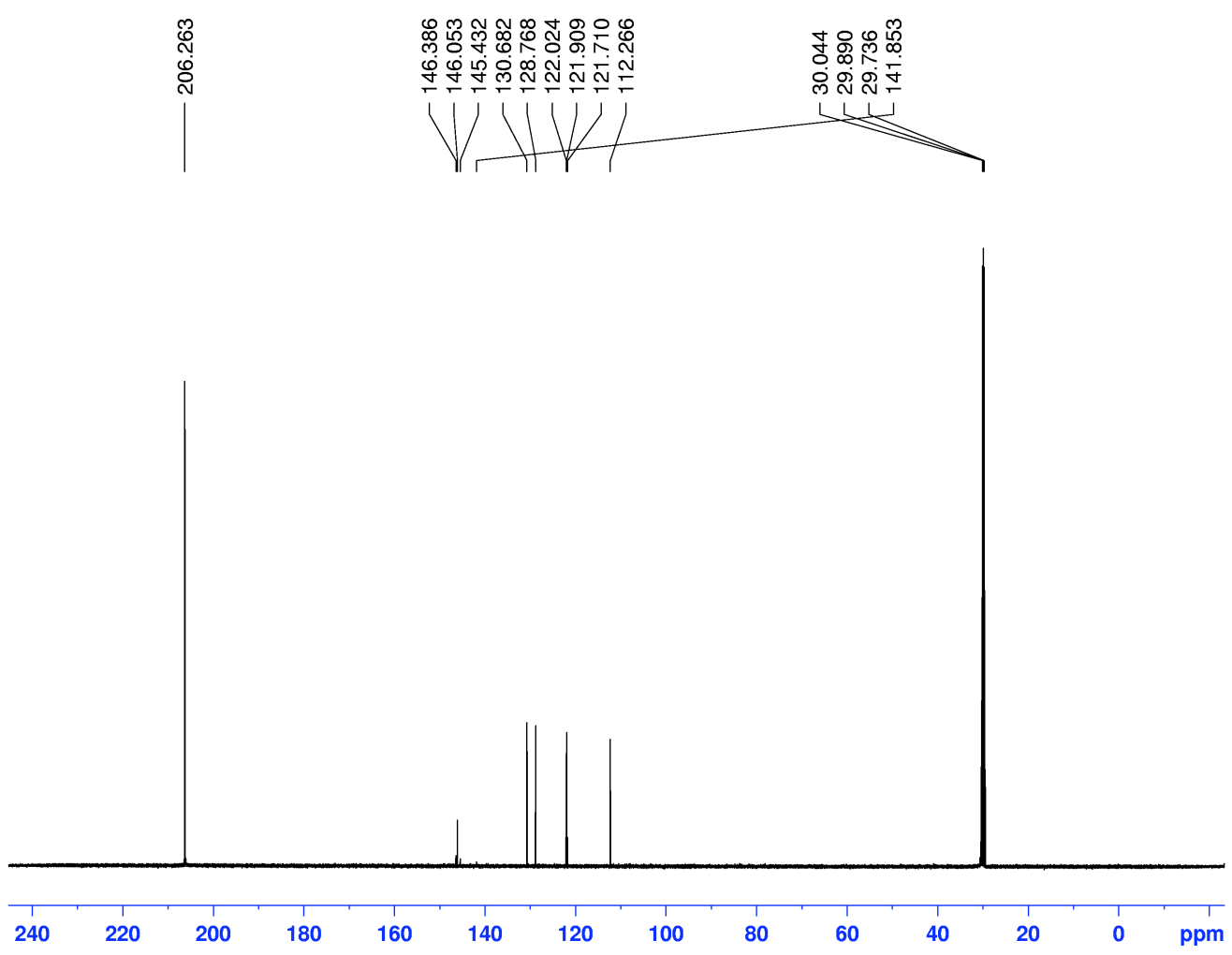
Comparison of benzene, nitrobenzene and dinitrobenzene 2-arylsulfenylpyrroles Jose R. Garabatos-Perera, Benjamin H. Rotstein and Alison Thompson*

\section{DEPTQ135 NMR spectrum, 2-(2,4-Dinitrophenylsulfenyl)-5-nitropyrrole}

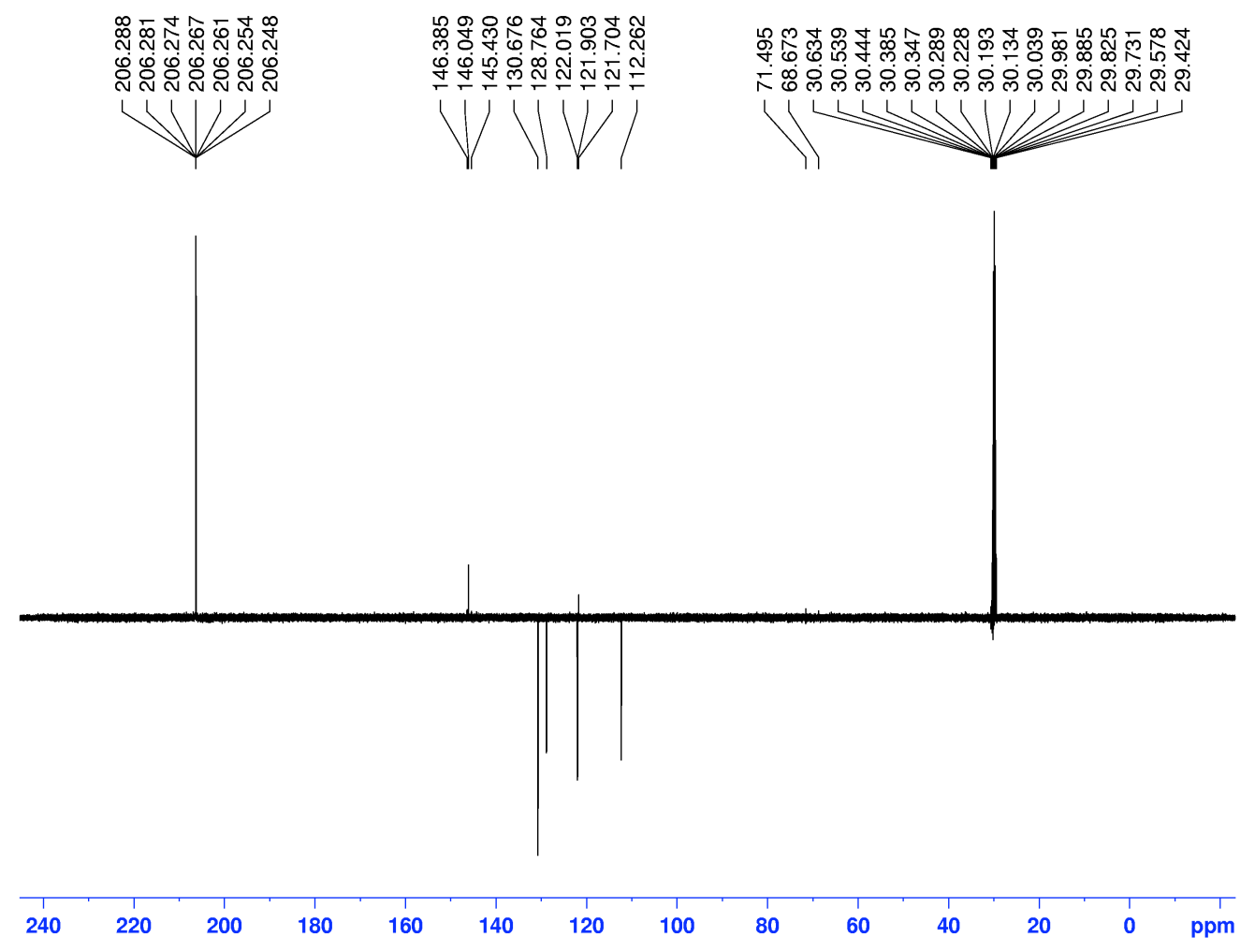


Comparison of benzene, nitrobenzene and dinitrobenzene 2-arylsulfenylpyrroles Jose R. Garabatos-Perera, Benjamin H. Rotstein and Alison Thompson*

\section{HMQC NMR spectrum, 2-(2,4-Dinitrophenylsulfenyl)-5-nitropyrrole}

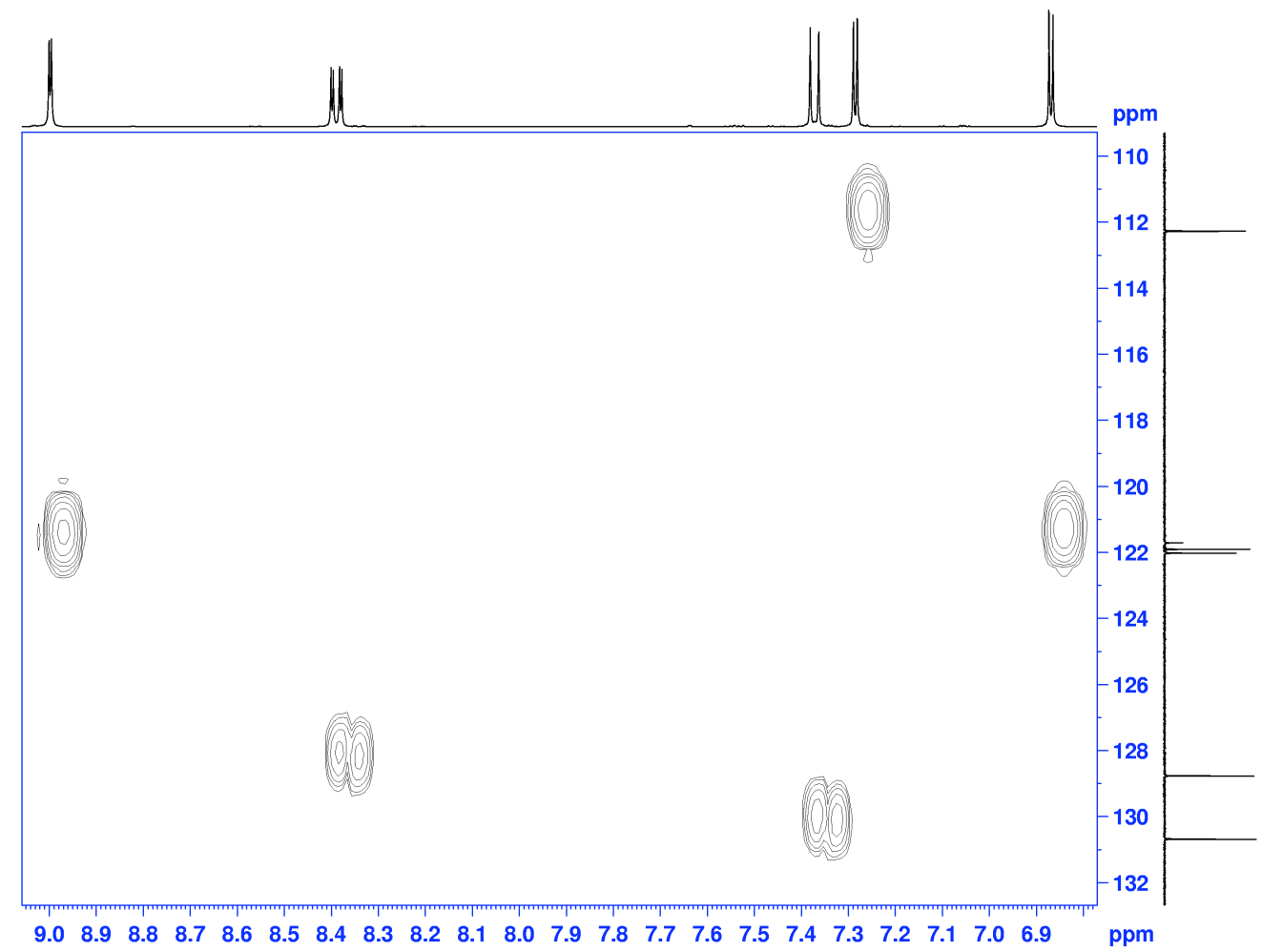


Comparison of benzene, nitrobenzene and dinitrobenzene 2-arylsulfenylpyrroles Jose R. Garabatos-Perera, Benjamin H. Rotstein and Alison Thompson*

\section{HMBC NMR spectrum, 2-(2,4-Dinitrophenylsulfenyl)-5-nitropyrrole}

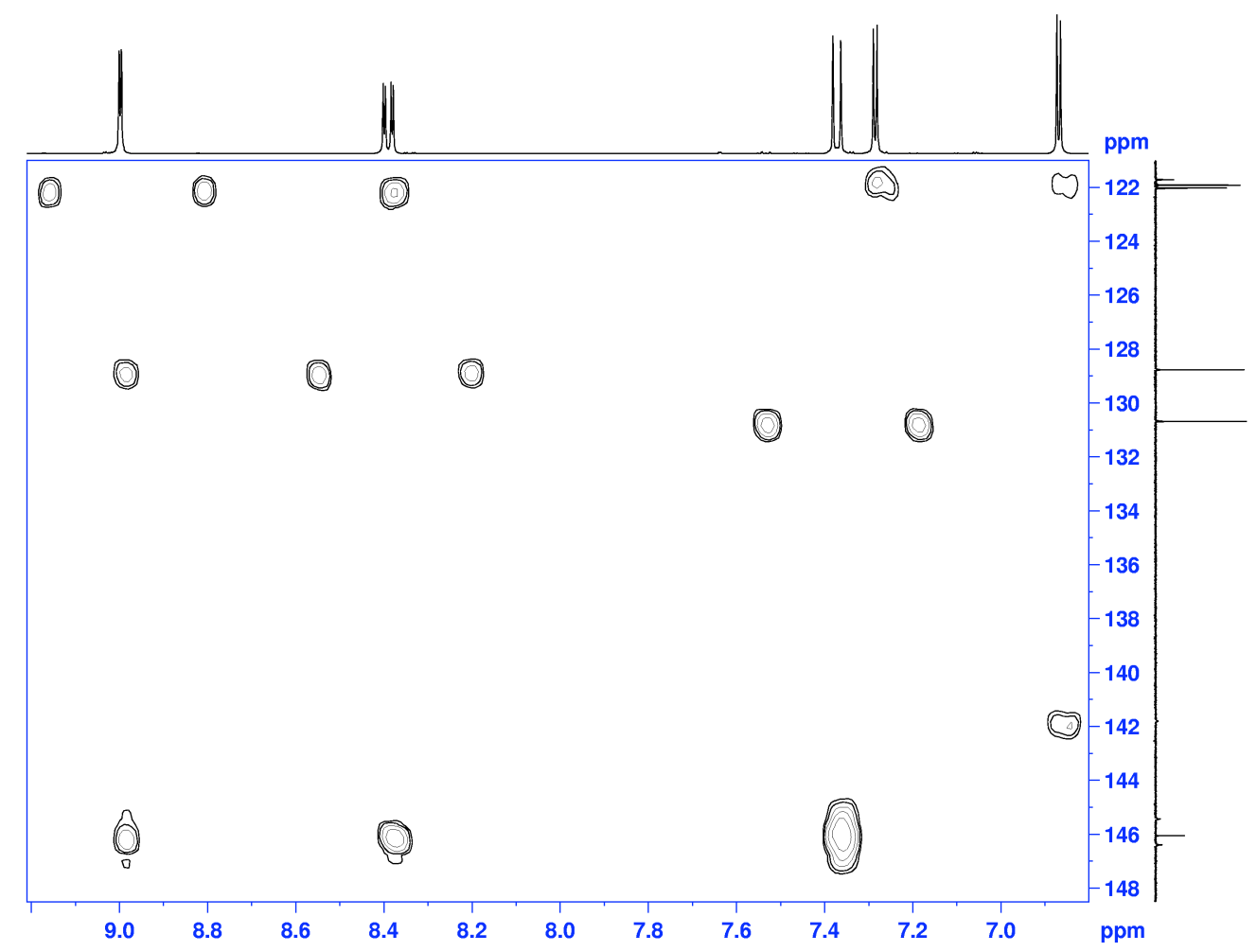


Comparison of benzene, nitrobenzene and dinitrobenzene 2-arylsulfenylpyrroles Jose R. Garabatos-Perera, Benjamin H. Rotstein and Alison Thompson*

Crude ${ }^{1} \mathrm{H}$ spectrum, 3-(4-Nitrobenzenesulfenyl)pyrrole and 2-(4Nitrobenzenesulfenyl)pyrrole (1:1)

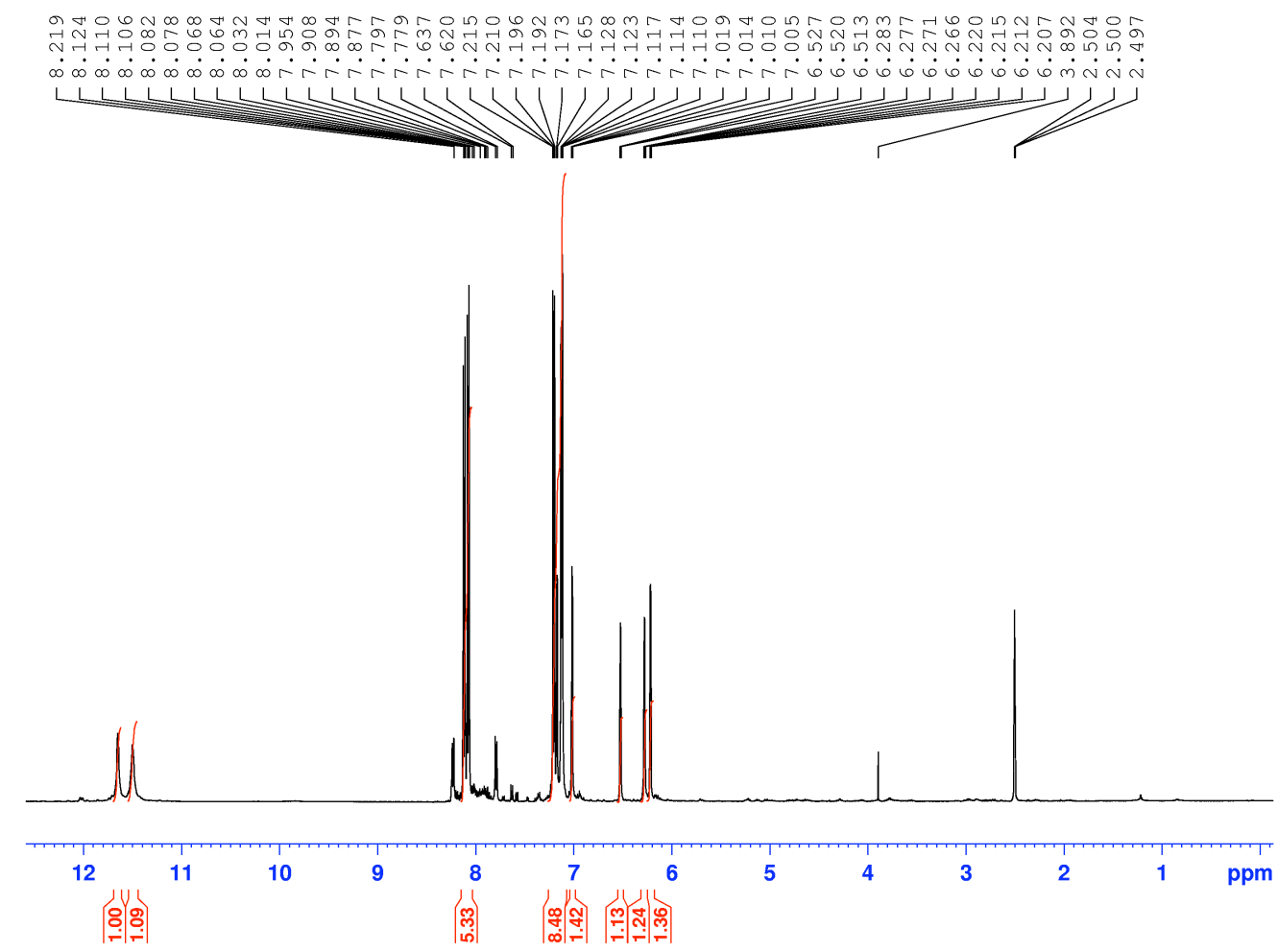


Comparison of benzene, nitrobenzene and dinitrobenzene 2-arylsulfenylpyrroles Jose R. Garabatos-Perera, Benjamin H. Rotstein and Alison Thompson*

Crude ${ }^{13} \mathrm{C}$ spectrum, 3-(4-Nitrobenzenesulfenyl)pyrrole and 2-(4Nitrobenzenesulfenyl)pyrrole

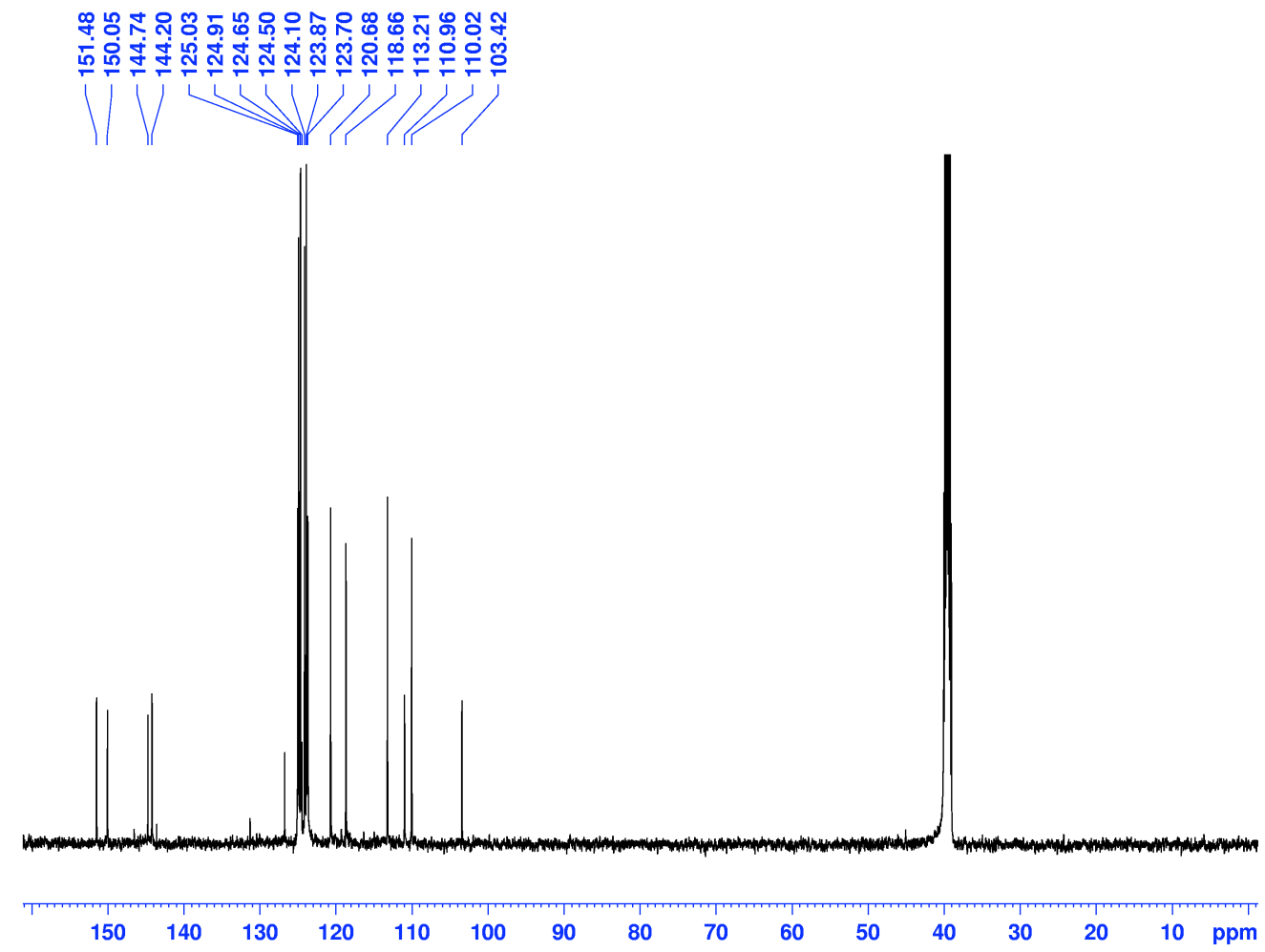


Comparison of benzene, nitrobenzene and dinitrobenzene 2-arylsulfenylpyrroles Jose R. Garabatos-Perera, Benjamin H. Rotstein and Alison Thompson*

Crude ${ }^{1} \mathrm{H}$ spectrum, 3-(2,4-Dinitrobenzenesulfenyl)pyrrole (major) and 2-(2,4Dinitrobenzenesulfenyl)pyrrole (1:4)

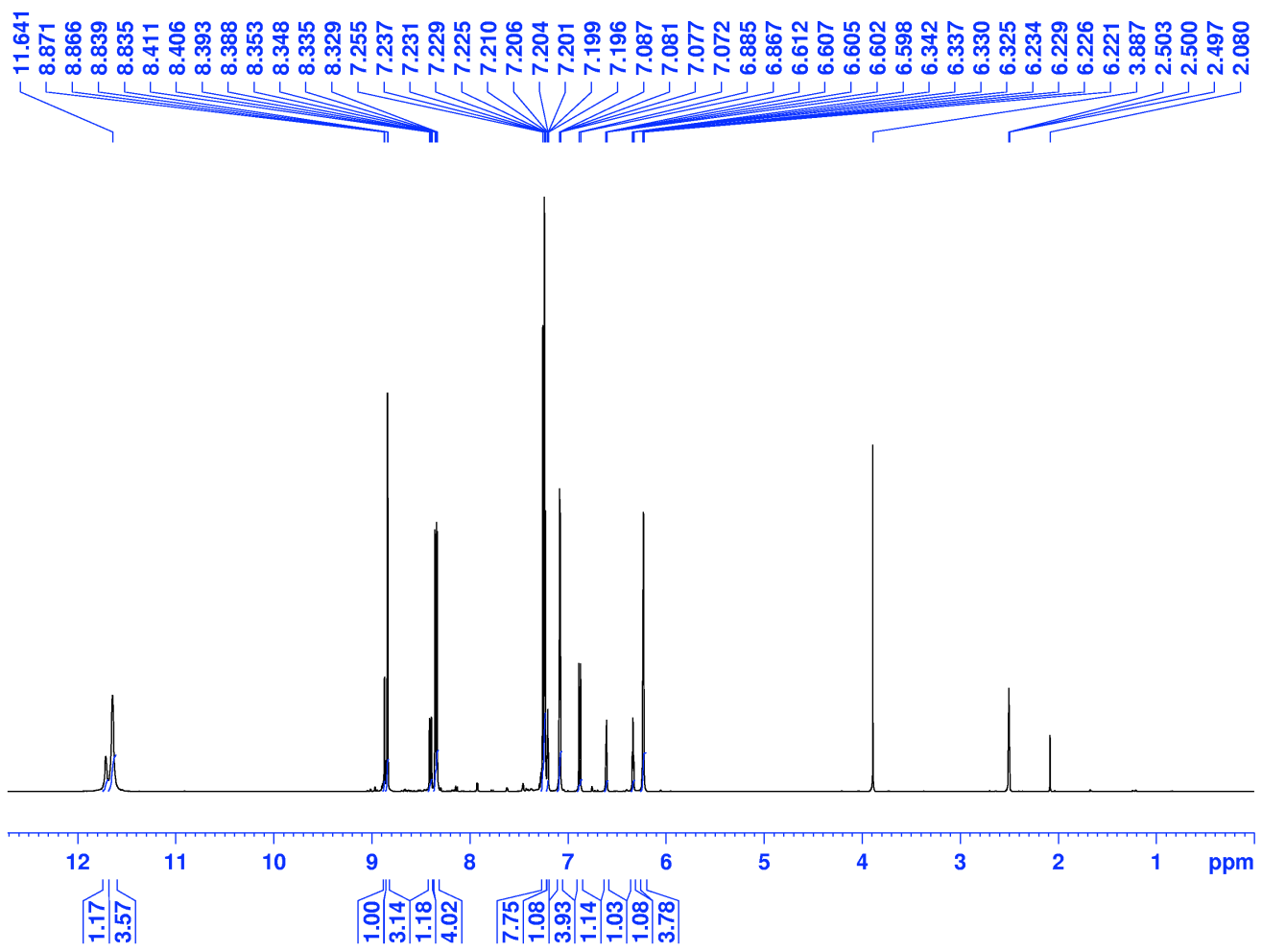


Comparison of benzene, nitrobenzene and dinitrobenzene 2-arylsulfenylpyrroles Jose R. Garabatos-Perera, Benjamin H. Rotstein and Alison Thompson*

Crude ${ }^{13} \mathrm{C}$ spectrum, 3-(2,4-Dinitrobenzenesulfenyl)pyrrole (major) and 2-(2,4Dinitrobenzenesulfenyl)pyrrole

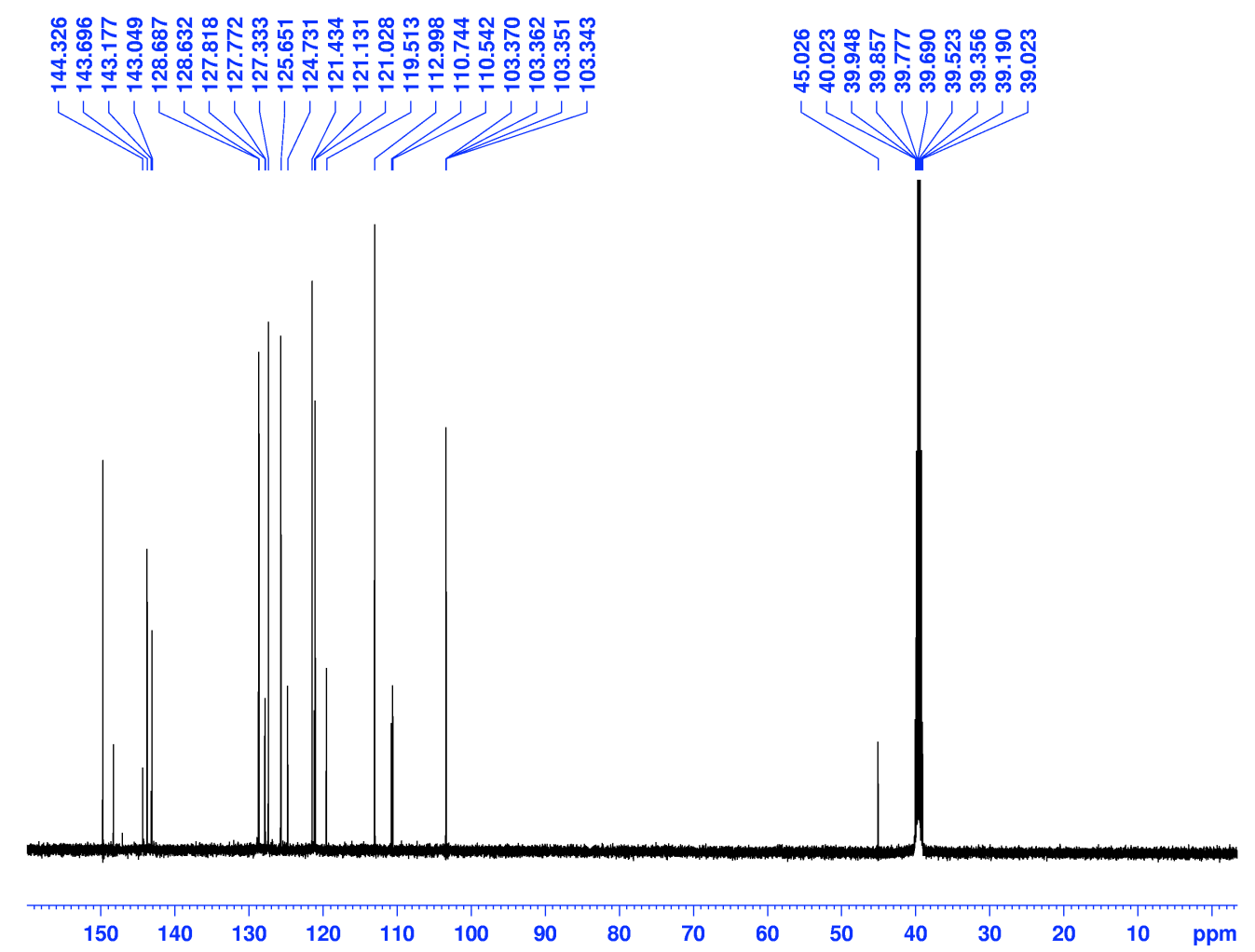


Comparison of benzene, nitrobenzene and dinitrobenzene 2-arylsulfenylpyrroles Jose R. Garabatos-Perera, Benjamin H. Rotstein and Alison Thompson*

Crude ${ }^{1} \mathbf{H}$ spectrum, 3-(4-Nitrobenzenesulfinyl)pyrrole (major) and 2-(4Nitrobenzenesulfinyl)pyrrole (1:6)

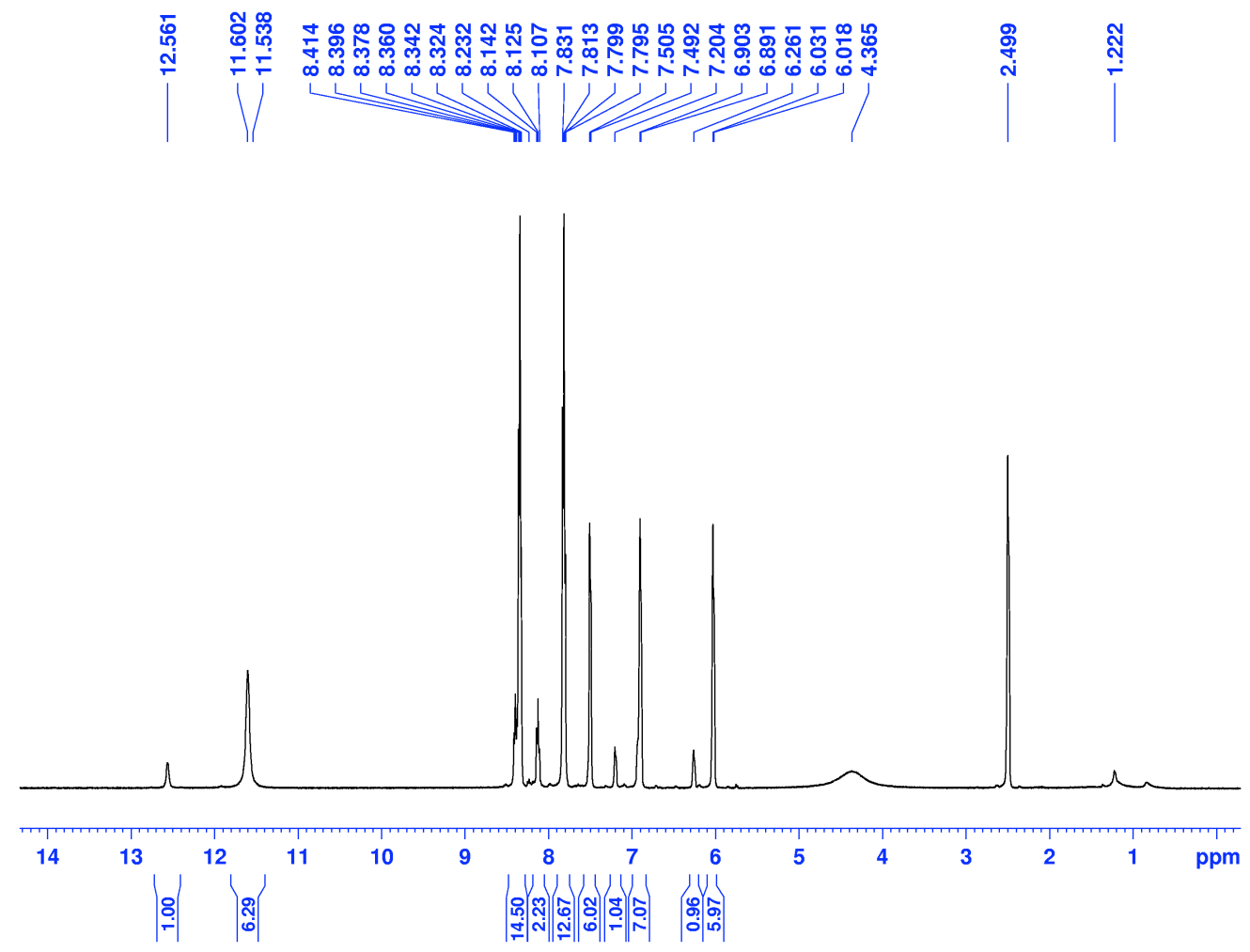


Comparison of benzene, nitrobenzene and dinitrobenzene 2-arylsulfenylpyrroles Jose R. Garabatos-Perera, Benjamin H. Rotstein and Alison Thompson*

Crude ${ }^{13} \mathrm{C}$ spectrum, 3-(4-Nitrobenzenesulfinyl)pyrrole (major) and 2-(4Nitrobenzenesulfinyl)pyrrole

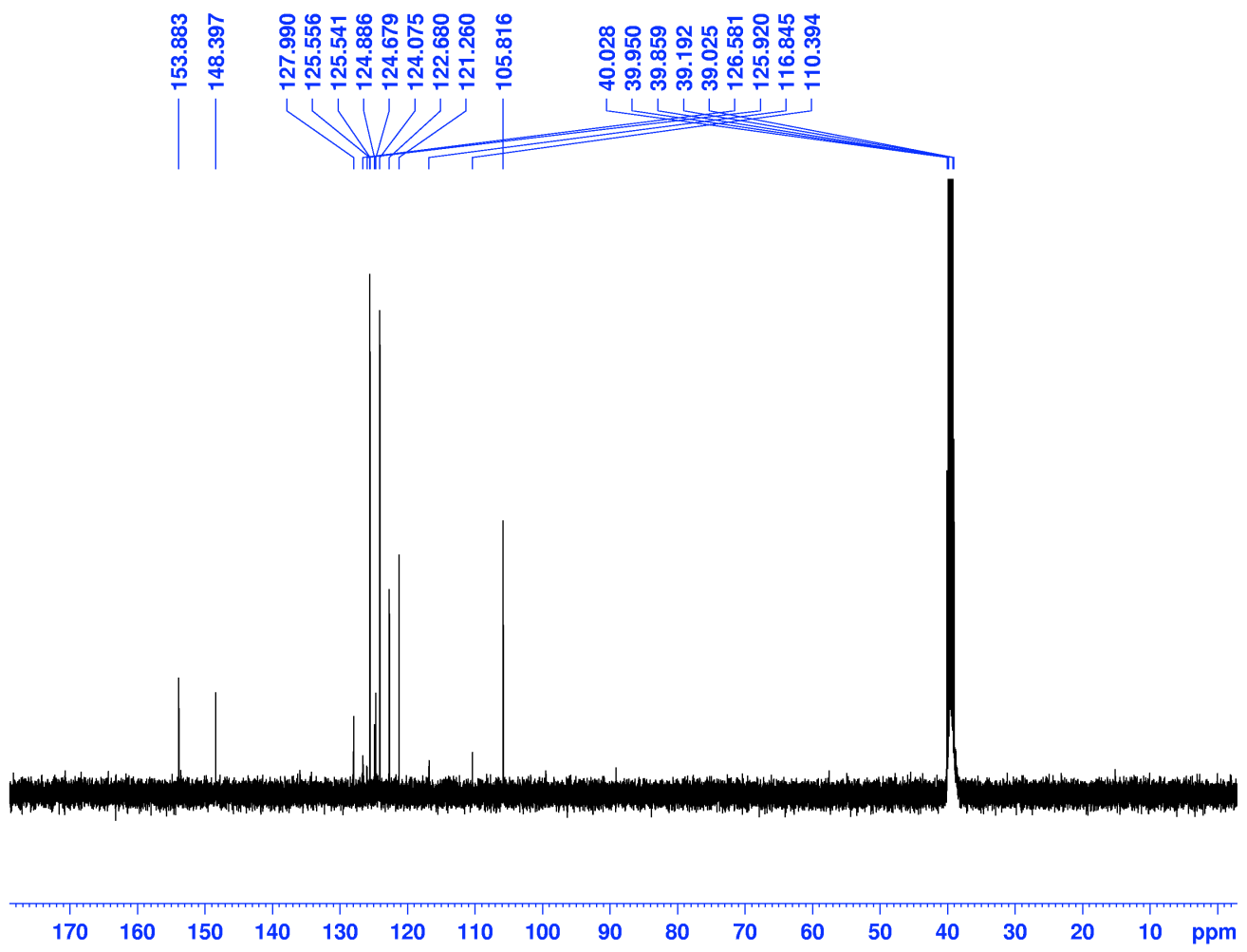


Comparison of benzene, nitrobenzene and dinitrobenzene 2-arylsulfenylpyrroles Jose R. Garabatos-Perera, Benjamin H. Rotstein and Alison Thompson*

${ }^{13} \mathrm{C}$ NMR spectrum, 1,9-Bis(2,4-dinitrobenzenesulfenyl)-5-phenyldipyrromethane

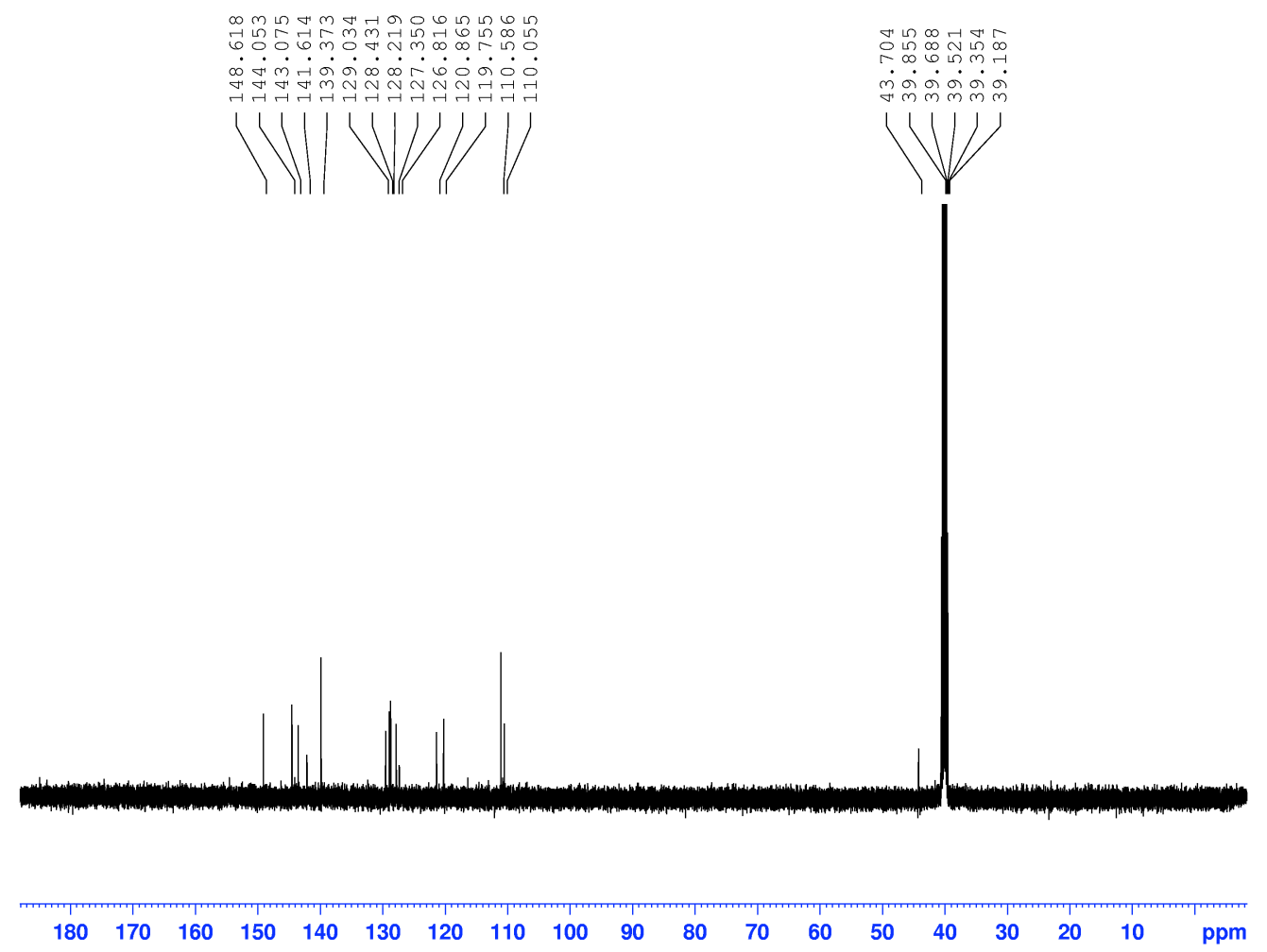


Comparison of benzene, nitrobenzene and dinitrobenzene 2-arylsulfenylpyrroles Jose R. Garabatos-Perera, Benjamin H. Rotstein and Alison Thompson*

\section{DEPTQ135 spectrum, 1,9-Bis(2,4-dinitrobenzenesulfenyl)-5-phenyldipyrromethane}

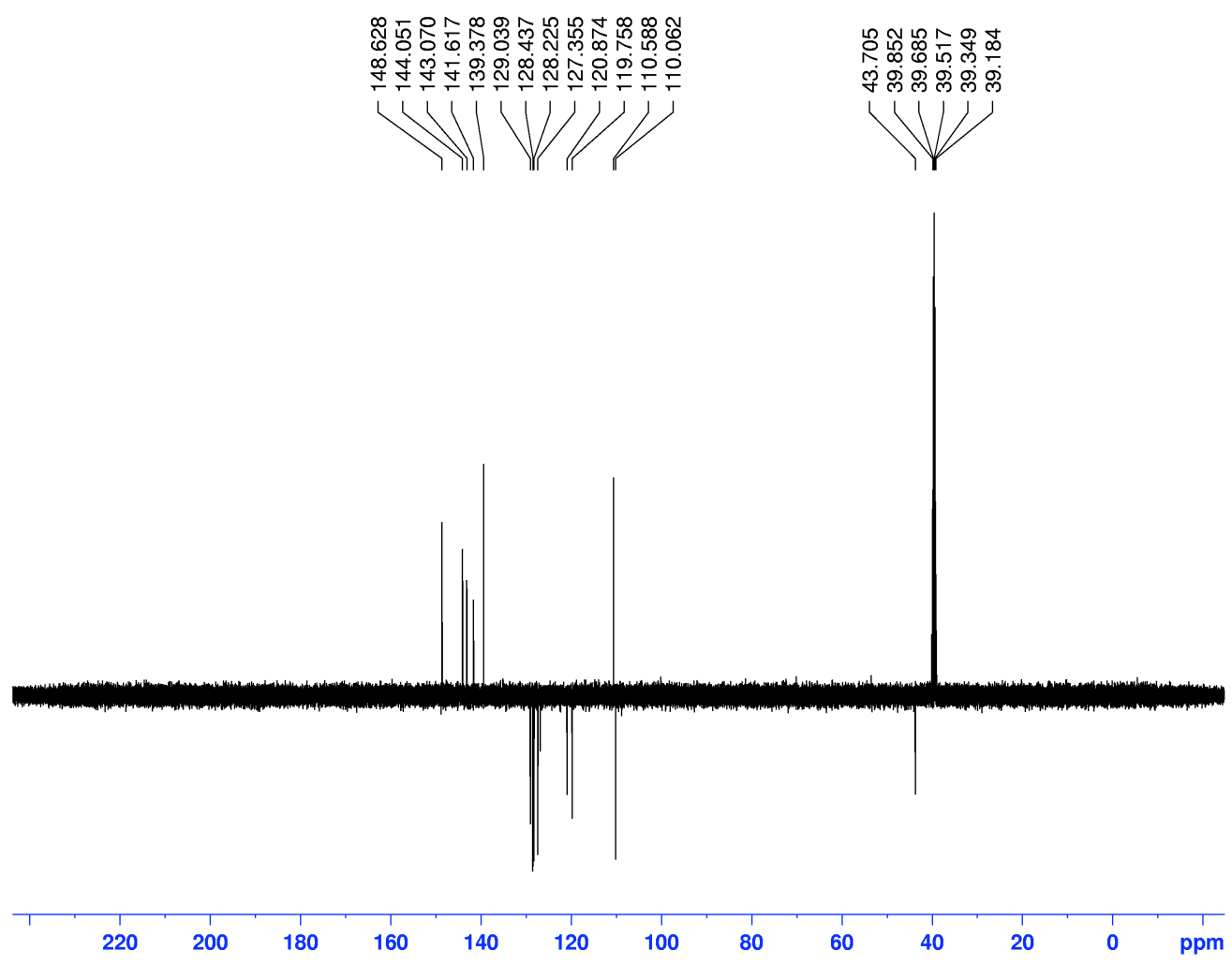


Comparison of benzene, nitrobenzene and dinitrobenzene 2-arylsulfenylpyrroles Jose R. Garabatos-Perera, Benjamin H. Rotstein and Alison Thompson*

\section{H M Q C NMR spectrum, 1,9-Bis(2,4-dinitrobenzenesulfenyl)-5- \\ phenyldipyrromethane}

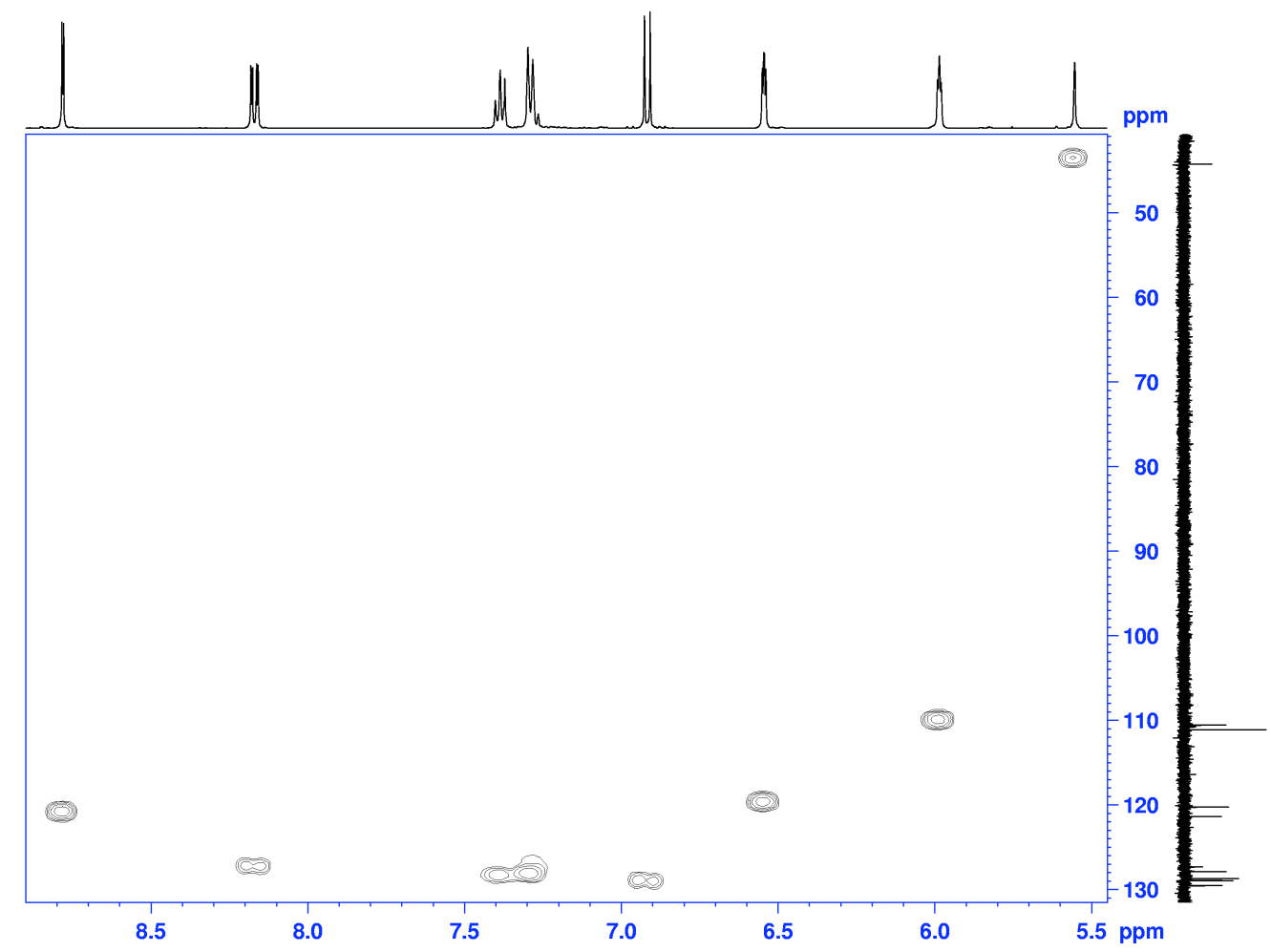


Comparison of benzene, nitrobenzene and dinitrobenzene 2-arylsulfenylpyrroles Jose R. Garabatos-Perera, Benjamin H. Rotstein and Alison Thompson*

\section{HMBC NMR spectrum, 1,9-B is (2,4-dinitrobenzenesulfenyl)-5- phenyldipyrromethane}

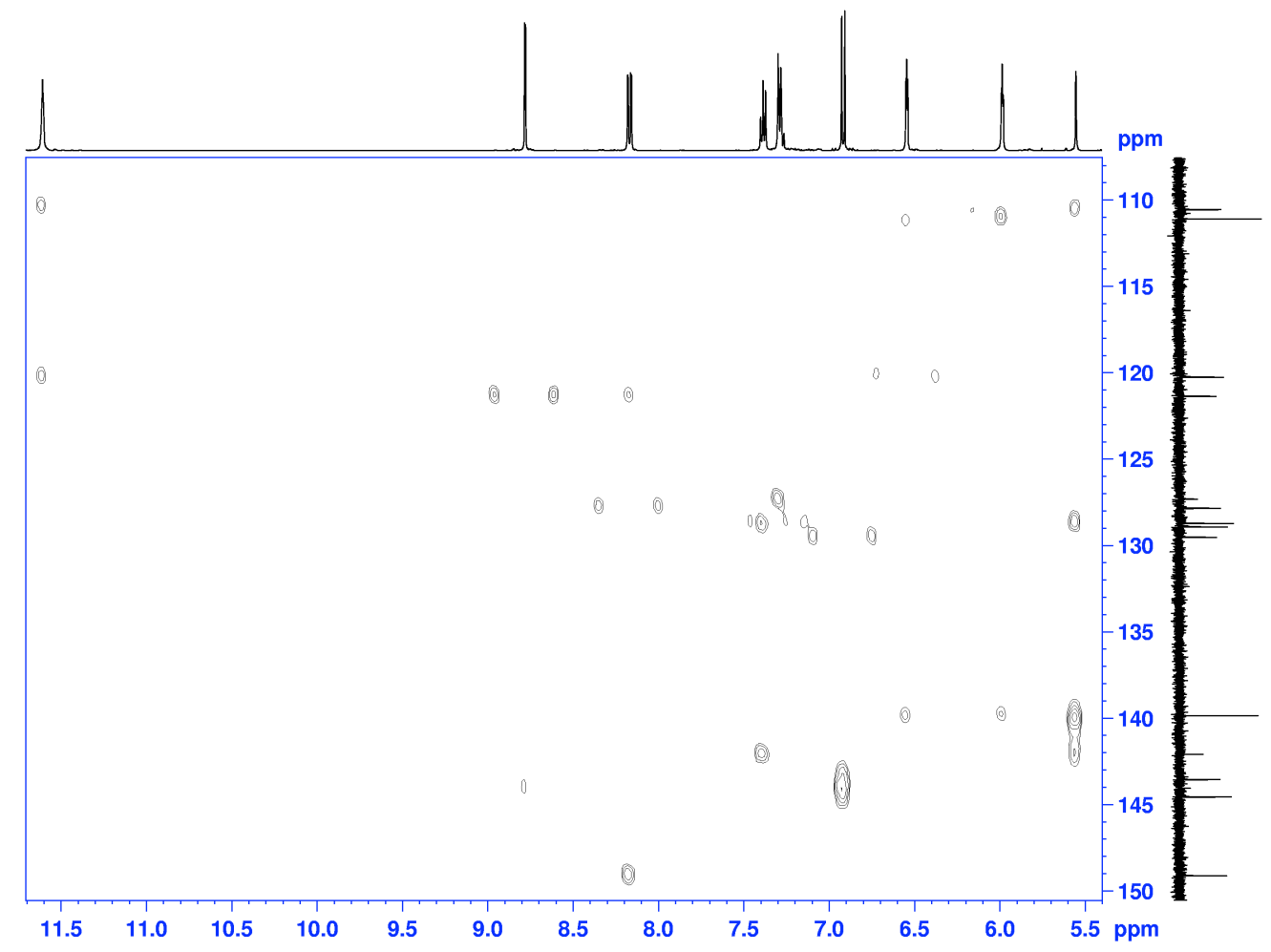


Comparison of benzene, nitrobenzene and dinitrobenzene 2-arylsulfenylpyrroles Jose R. Garabatos-Perera, Benjamin H. Rotstein and Alison Thompson*

\section{${ }^{13} \mathrm{C} \quad$ NMR spectru m, 1,9 - B is ( 2,4 - d in it r obenzenesulfenyl)-5-(4- methylphenyl)dipyrromethane}

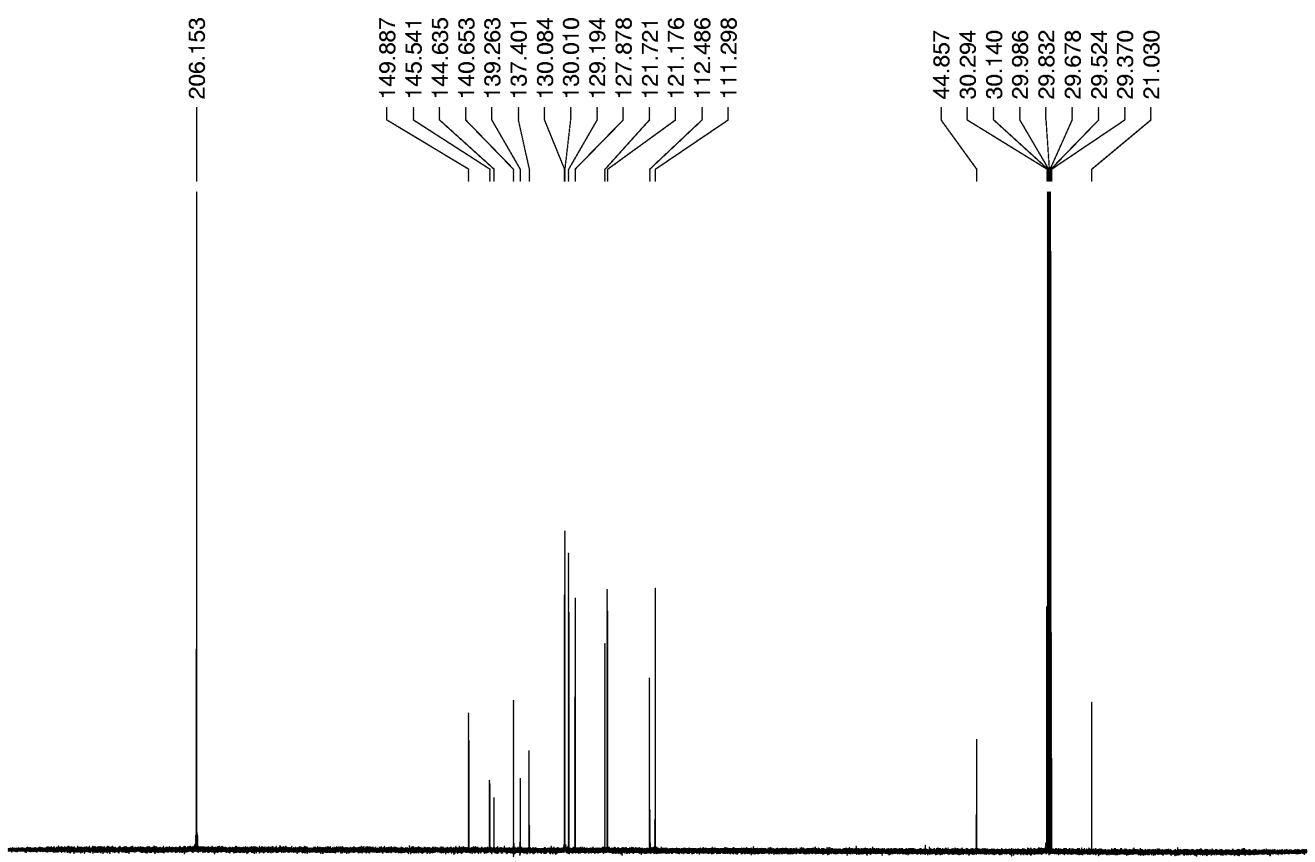

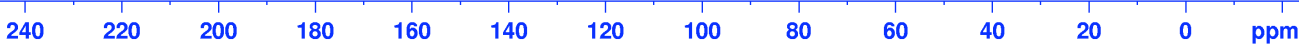


Comparison of benzene, nitrobenzene and dinitrobenzene 2-arylsulfenylpyrroles Jose R. Garabatos-Perera, Benjamin H. Rotstein and Alison Thompson*

\section{DEPTQ135 NMR spectrum, 1,9-Bis(2,4-dinitrobenzenesulfenyl)-5-(4-}

methylphenyl)-dipyrromethane

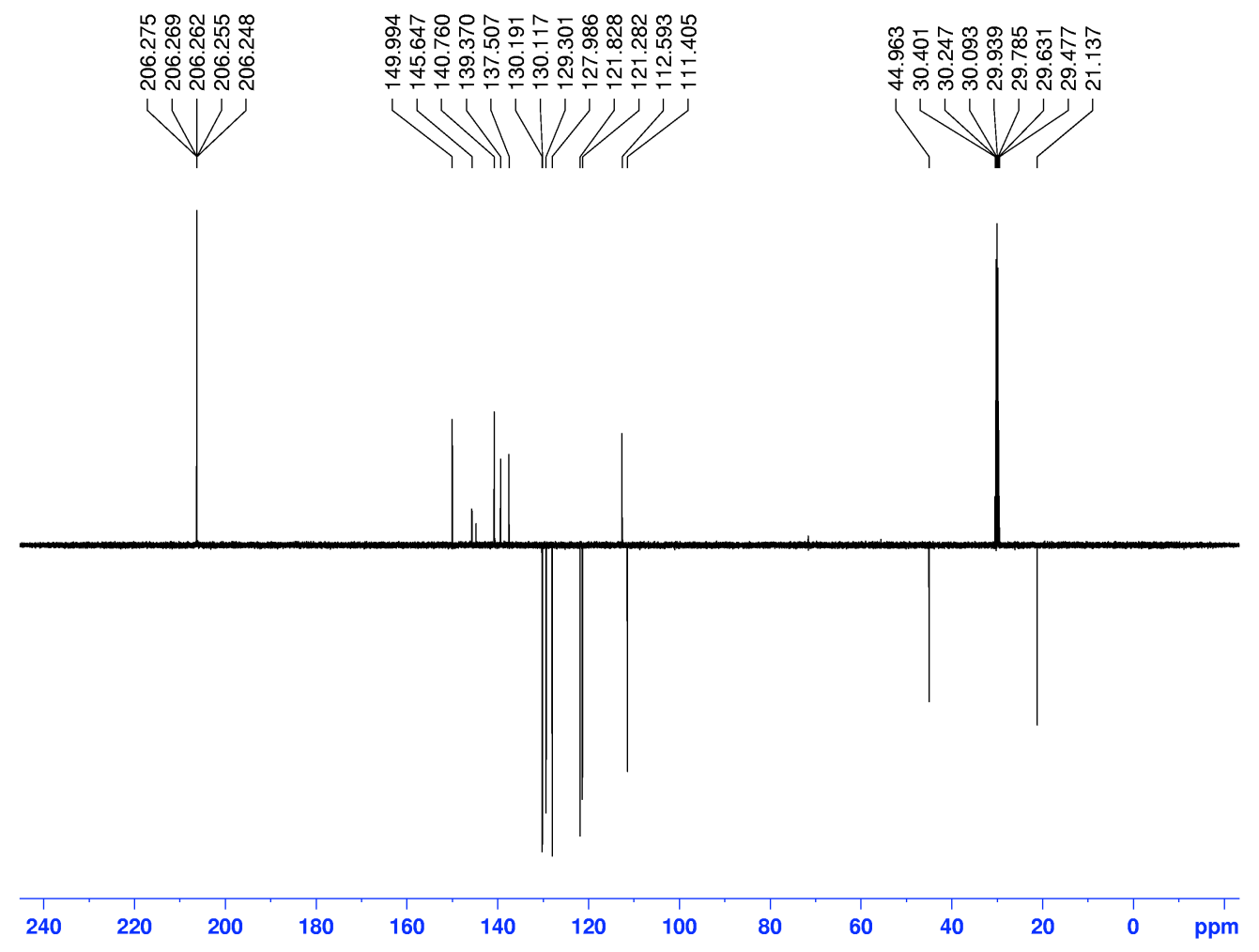


Comparison of benzene, nitrobenzene and dinitrobenzene 2-arylsulfenylpyrroles Jose R. Garabatos-Perera, Benjamin H. Rotstein and Alison Thompson*

HMQC NMR spectrum, 1,9-Bis(2,4-dinitrobenzenesulfenyl)-5-(4-methylphenyl)dipyrromethane

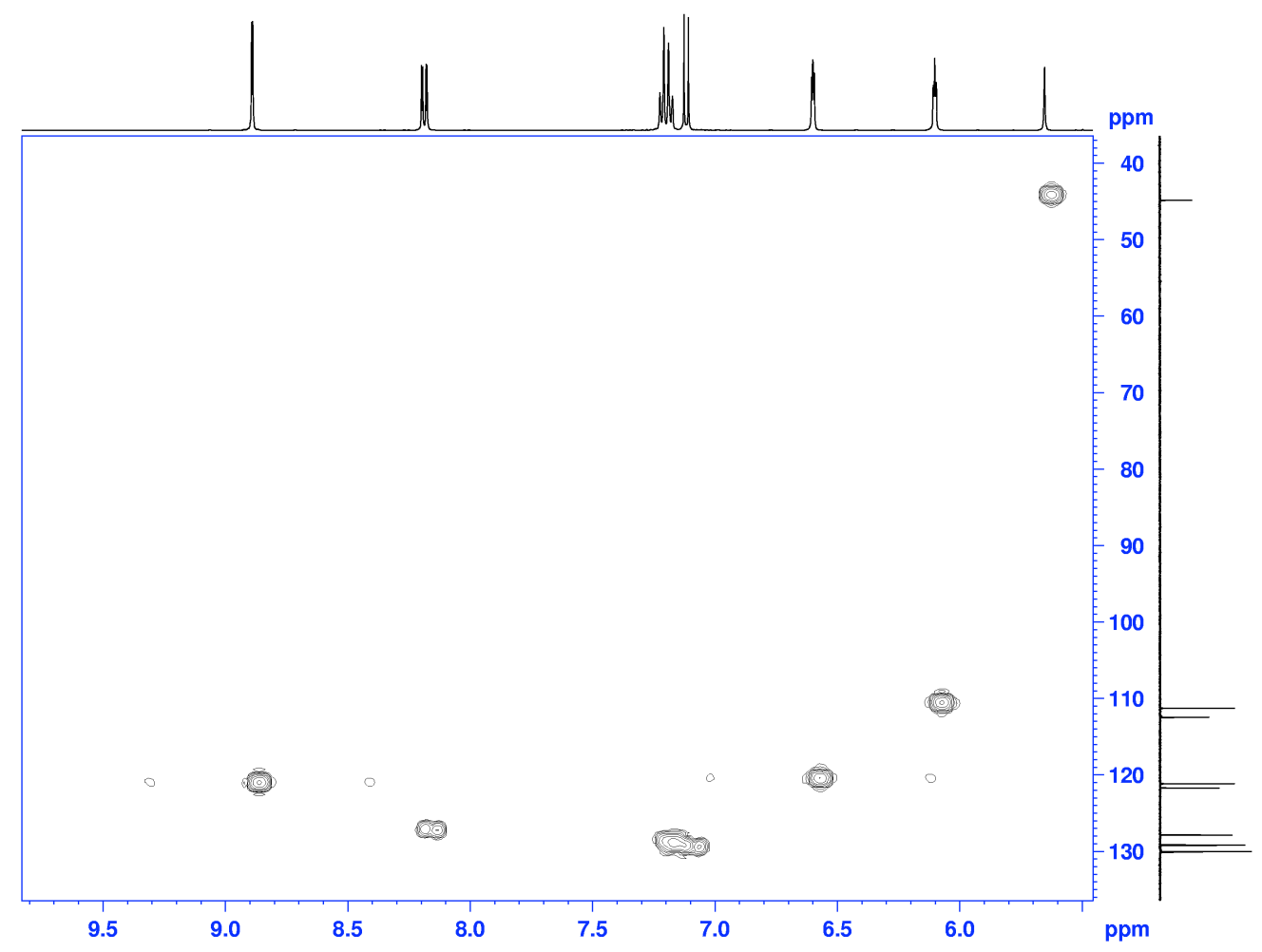


Comparison of benzene, nitrobenzene and dinitrobenzene 2-arylsulfenylpyrroles Jose R. Garabatos-Perera, Benjamin H. Rotstein and Alison Thompson*

HMBC NMR spectrum, 1,9-Bis(2,4-dinitrobenzenesulfenyl)-5-(4-methylphenyl)dipyrromethane

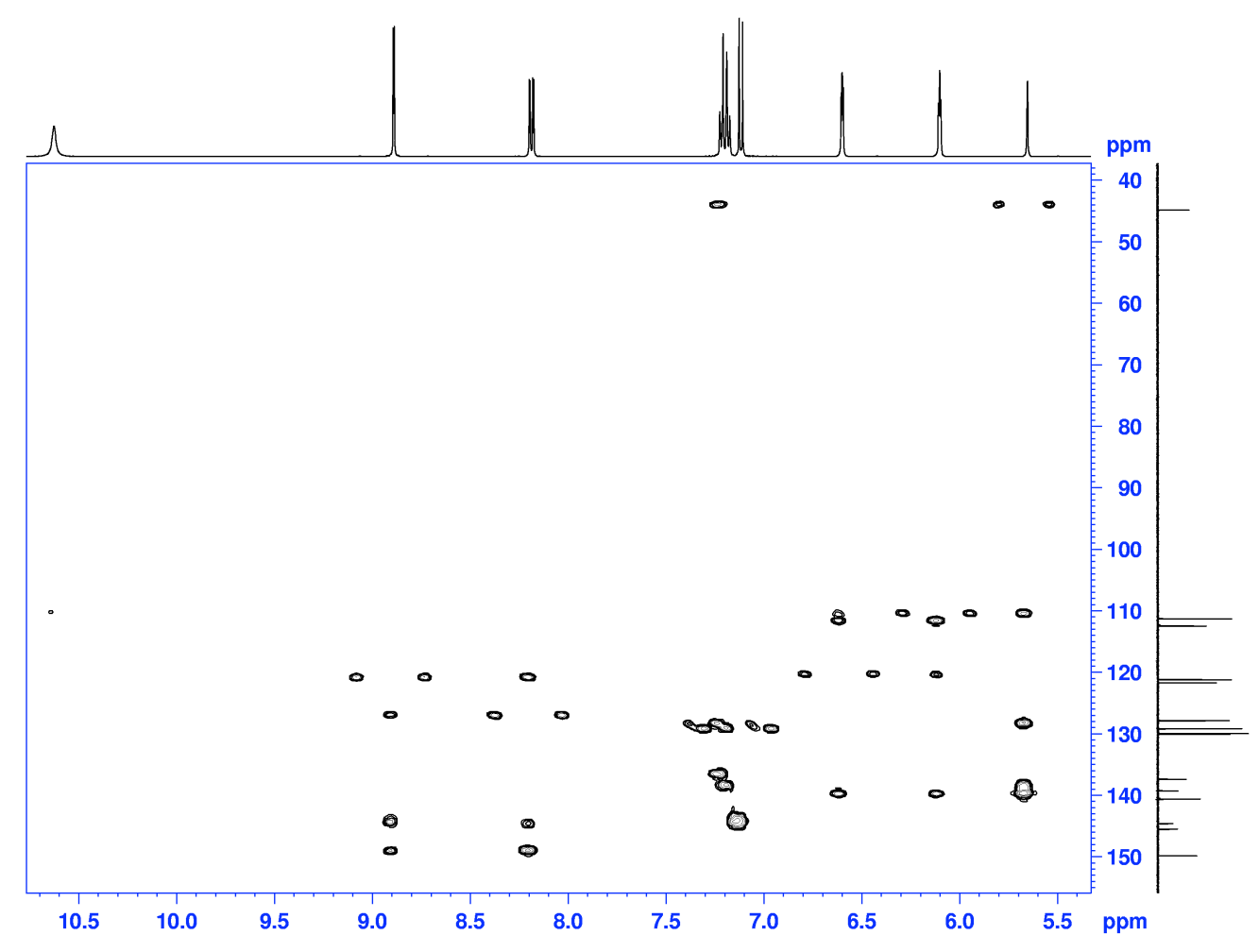


Comparison of benzene, nitrobenzene and dinitrobenzene 2-arylsulfenylpyrroles Jose R. Garabatos-Perera, Benjamin H. Rotstein and Alison Thompson*

\section{${ }^{13} \mathrm{C} \quad \mathrm{NMR} \quad$ spectrum, 1,9-B is (2,4 - d in it r obenzenesulfenyl)-5-(4- nitrophenyl)dipyrromethane}

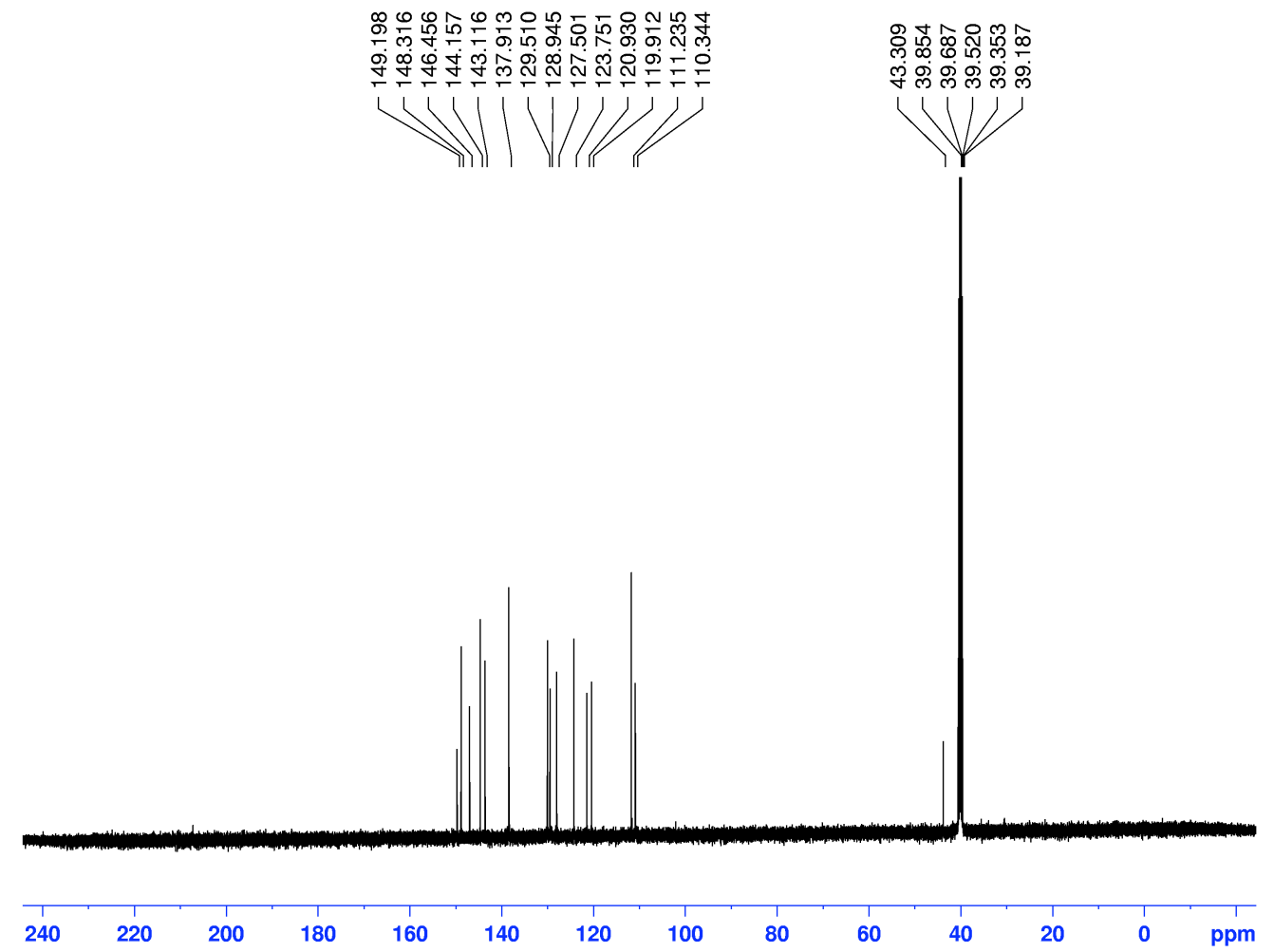


Comparison of benzene, nitrobenzene and dinitrobenzene 2-arylsulfenylpyrroles Jose R. Garabatos-Perera, Benjamin H. Rotstein and Alison Thompson*

DEPTQ135 NMR spectrum, 1,9-Bis(2,4-dinitrobenzenesulfenyl)-5-(4-nitrophenyl)dipyrromethane

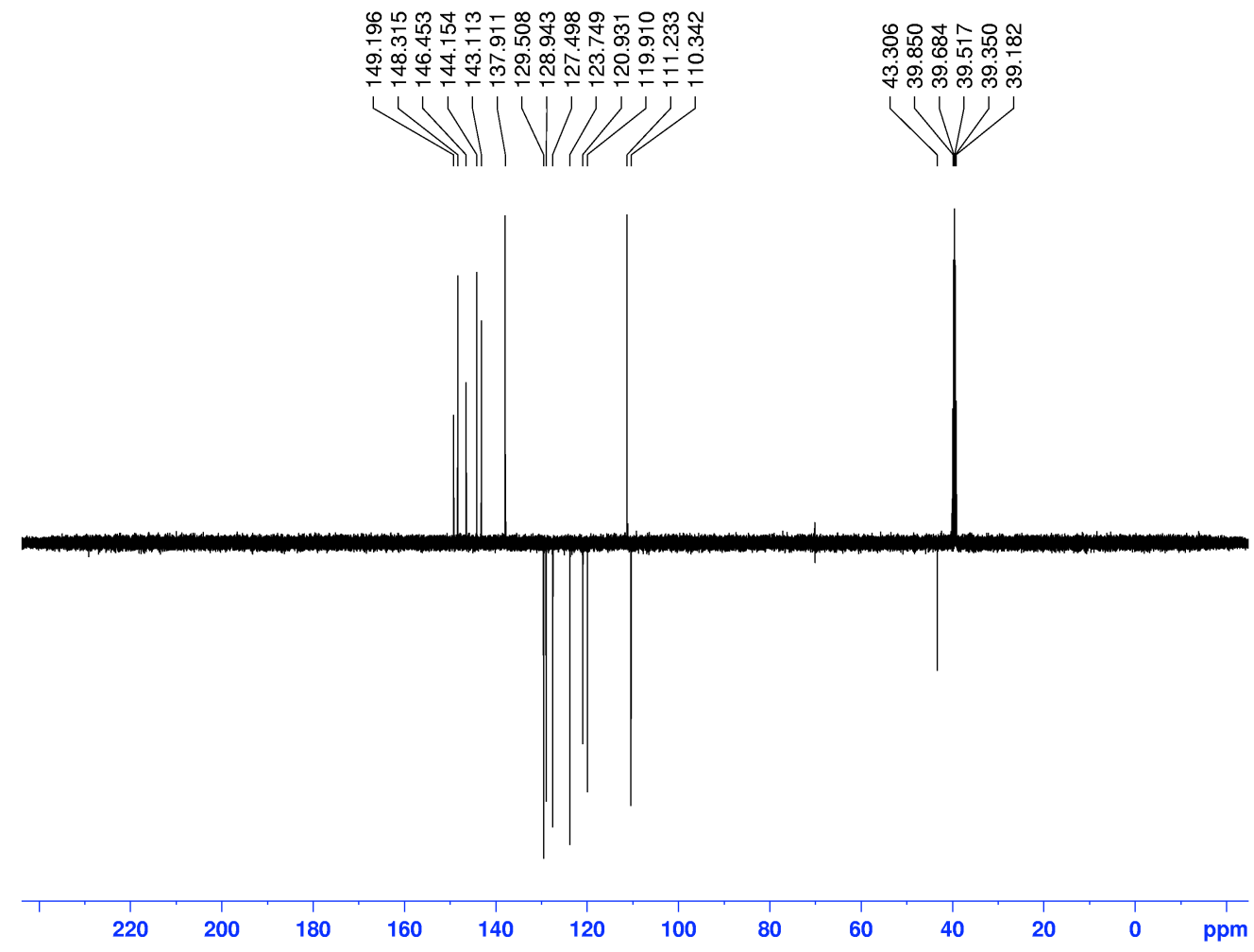


Comparison of benzene, nitrobenzene and dinitrobenzene 2-arylsulfenylpyrroles Jose R. Garabatos-Perera, Benjamin H. Rotstein and Alison Thompson*

HMQC NMR spectrum, 1,9-Bis(2,4-dinitrobenzenesulfenyl)-5-(4-nitrophenyl)dipyrromethane

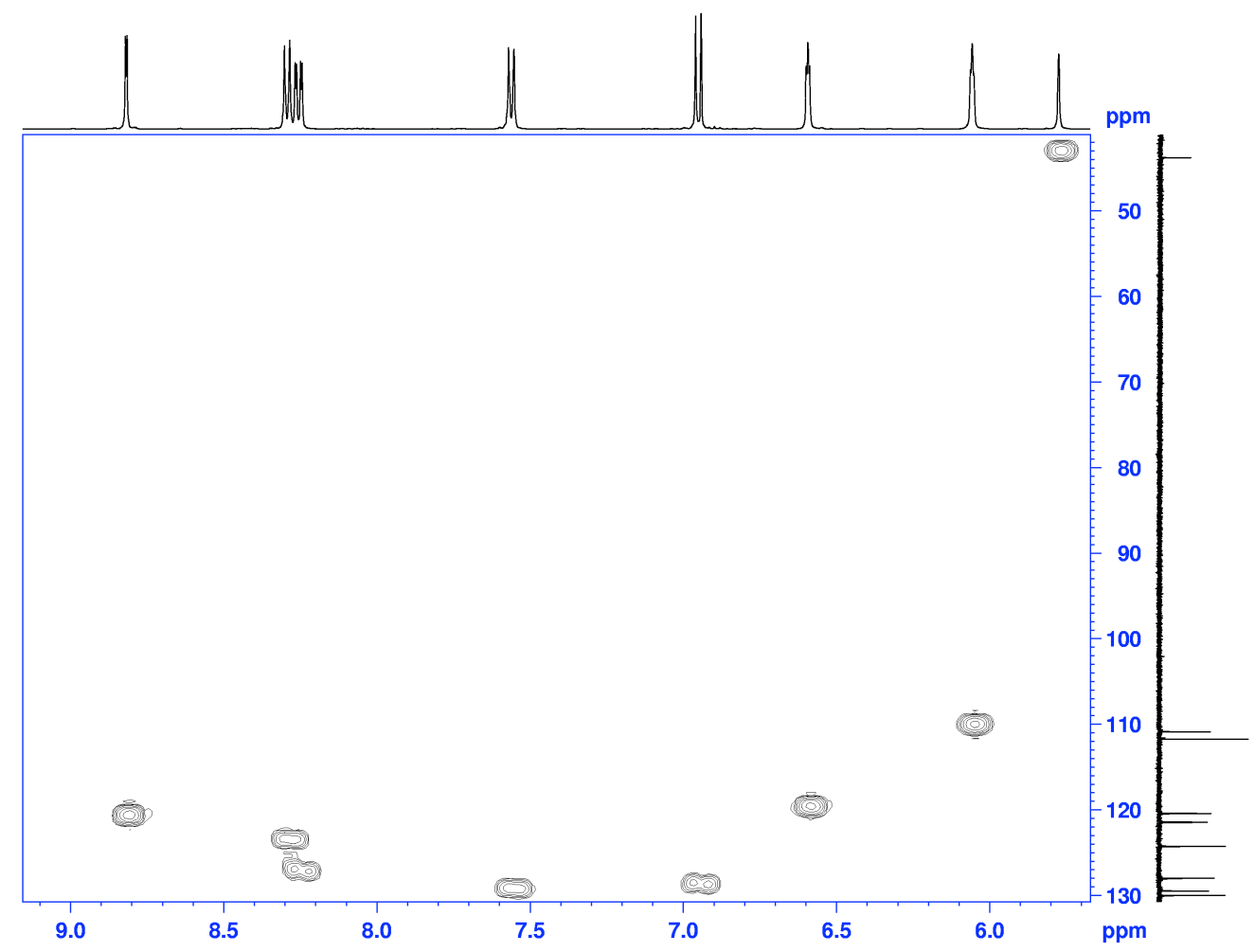


Comparison of benzene, nitrobenzene and dinitrobenzene 2-arylsulfenylpyrroles Jose R. Garabatos-Perera, Benjamin H. Rotstein and Alison Thompson*

HMBC NMR spectrum, 1,9-Bis(2,4-dinitrobenzenesulfenyl)-5-(4-nitrophenyl)dipyrromethane

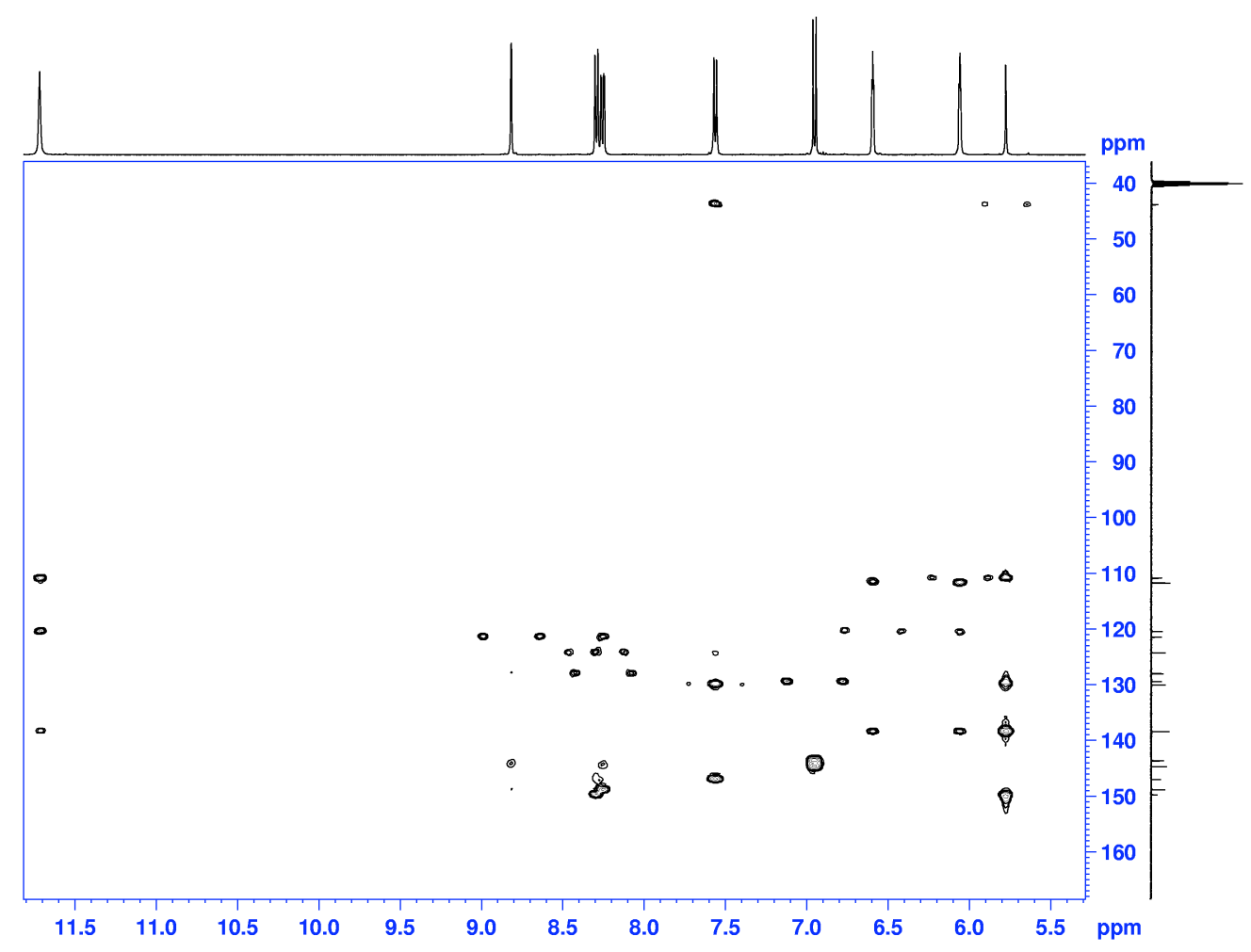


Comparison of benzene, nitrobenzene and dinitrobenzene 2-arylsulfenylpyrroles Jose R. Garabatos-Perera, Benjamin H. Rotstein and Alison Thompson*

\section{${ }^{13} \mathrm{C} \quad \mathrm{NMR} \quad \mathrm{spect}$ rum, 1,9-B is ( 2, 4 - d in it r obenzenesulfenyl)-5-(4- bromophenyl)dipyrromethane}

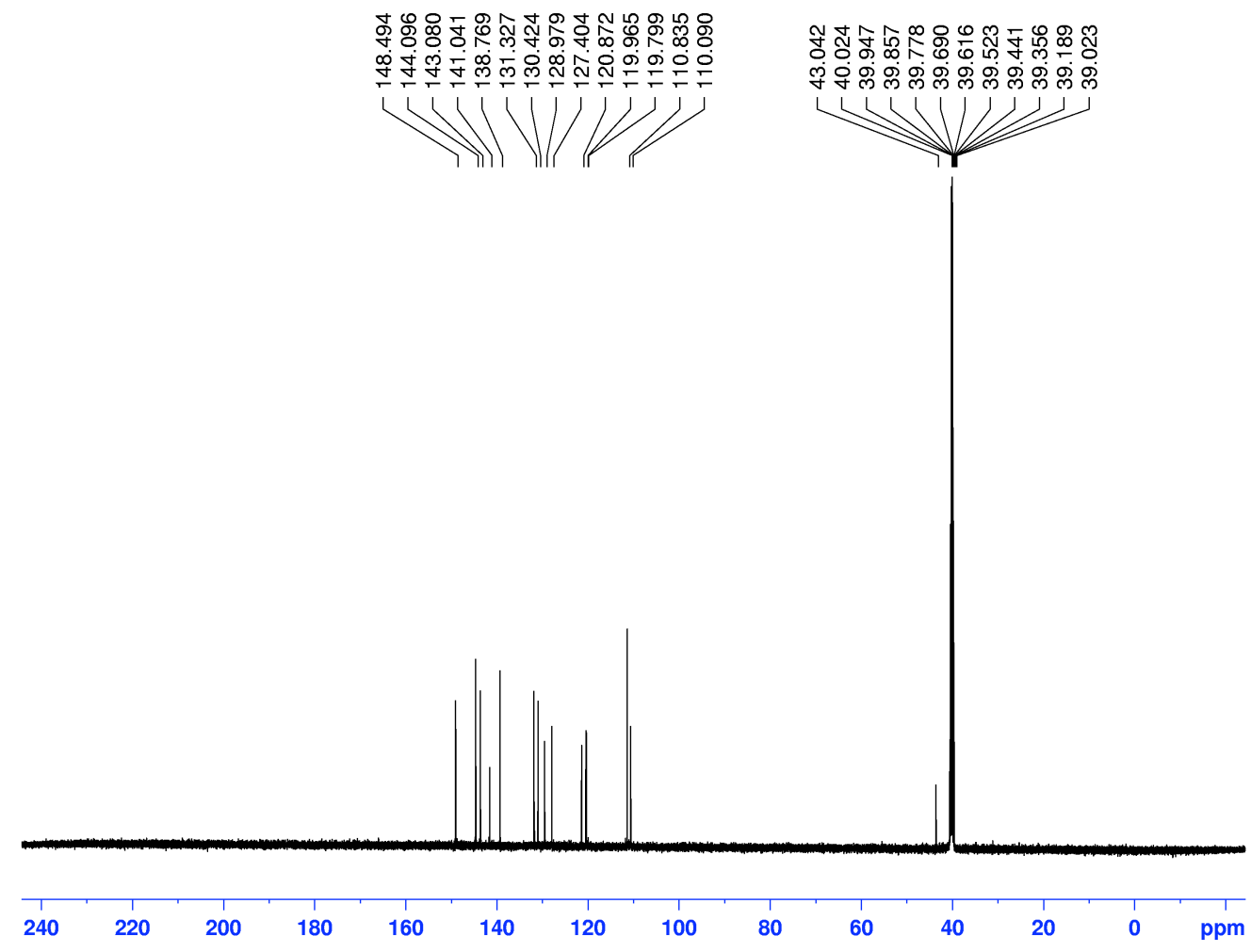


Comparison of benzene, nitrobenzene and dinitrobenzene 2-arylsulfenylpyrroles

Jose R. Garabatos-Perera, Benjamin H. Rotstein and Alison Thompson*

\section{$\underline{\text { References }}$}

(1) Campiani, G.; Nacci, V.; Bechelli, S.; Ciani, S. M.; Garofalo, A.; Fiorini, I.;

Wikstrom, H.; de Boer, P.; Liao, Y.; Tepper, P. G.; Cagnotto, A.; Mennini, T. J. Med. Chem. 1998, 41, 3763-3772.

(2) Thamyongkit, P.; Bhise, A. D.; Taniguchi, M.; Lindsey, J. S. J. Org. Chem. 2006, 71, 903-910.

(3) Thompson, A.; Butler, R. J.; Grundy, M. N.; Laltoo, A. B. E.; Robertson, K. N.; Cameron, T. S. J. Org. Chem. 2005, 70, 3753-3756.

(4) Zonta, C.; Fabris, F.; Lucchi, O. D. Org. Lett. 2005, 7, 1003-1006.

(5) Kakushima, M.; Frenette, R. J. J. Org. Chem. 1984, 49, 2025-2027.

(6) Carmona, O.; Greenhouse, R.; Landeros, R.; Muchowski, J. M. J. Org. Chem. 1980, 45, 5336-5339.

(7) Muchowski, J. M.; Hess, P. Tetrahedron Lett. 1988, 29, 777-780. 